\title{
Return Spillover across Bitcoin Markets and Foreign Exchange Pairs Dominated in major Trading Currencies
}

\begin{abstract}
Muhammad Owais Qarni ${ }^{1}$, Saqib Gulzar ${ }^{2}$
Abstract

This study examines the dynamic nature of return spillover across Bitcoins indices and foreign exchange pairs denominated in 6 major trading currencies. The findings of spillover index, Spillover Asymmetry Measure (SAM) and frequency connectedness methodologies indicate that return spillover across Bitcoin markets and foreign exchange pairs dominated in six major trading currencies is very low. The intra-market return spillover for the Bitcoin markets and foreign exchange pairs is found to be significant. Presence of asymmetry in the return spillover is also found. Evidence indicates that return spillover are dominated in short horizon, with significant spillover occurring within 4 days of an event. The low integration of Bitcoin markets with the foreign exchange markets provide significant implication for portfolio diversification and risk minimization.
\end{abstract}

Keyword: Bitcoin, spillover index, major trading currencies, spillover asymmetry measure, frequency connectedness

JEL Classification: G10, G15, G19

\section{Introduction}

Financial integration is a process in which the financial markets of an economy becomes more closely unified with financial markets in other economies thus equalizing the returns on financial assets (Brouwer, 2005; Qarni \& Gulzar, 2018). Financial liberalisation and deregulation of financial markets around the world since mid-1980s has enhanced the integration of financial markets. As a result of financial markets' integration, accumulation of massive foreign positions occurred that affected exchange rate valuation (Ho, 2009). The world has witnessed the harmful effects of

1 PhD Scholar, Department of Management Sciences, COMSATS University Islamabad, Wah Campus, GT Road, Wah Cantt, Pakistan.Email: djl.tsri@gmail.com

2 Associate Professor of Finance, Department of Management Sciences, COMSATS University Islamabad, Wah Campus, GT Road, Wah Cantt, Pakistan. Email: saqibgulzar@ciitwah.edu.pk

\section{ARTICLE HISTORY}

12 Jul, 2019 Submission Received

03 Nov, 2019 First Review

24 Jan, 2020 Second Review

14 Mar, 2020 Accepted 
such financial integration due to global imbalances during the Asian financial crisis, Global financial crisis etc.

Measuring spillovers from Bitcoin to other financial markets is important to understand its portfolio diversification capabilities and its ability to withstand financial downturns. Regarding the price determinants of Bitcoin, researchers have found that Bitcoin prices exhibit detached behaviour that cannot be explained by economic fundamentals. Kristoufek (2015) proposed that the unexplained nature of Bitcoin price by economic theory makes it a pure speculative investment asset, where the main driver for price formation is investors' speculations. Similarly, Ciaian, Rajcaniova and Kancs (2016) found that investor attractiveness is the main drivers of Bitcoin prices, and there is no evidence that macro-financial developments have any impact on Bitcoin prices in the long run. With presence of unrelated price and return movement from conventional financial assets, Bitcoin is a sure choice for portfolio diversification and risk minimization by international investors.

Bitcoin is gaining importance as an investment alternative due to similar portfolio diversification characteristics like alternative investment instruments. Brandvold, Molnár, Vagstad, and Valstad (2015) found that Currency of trade for Bitcoin is an important determinant for the price difference at different alternative currency's Bitcoin exchanges, which is most likely to affect the foreign exchange rate (Narayan, Narayan, Rahman, \& Setiawan, 2018). The presence of diversity among the Bitcoin prices at different alternative currencies' exchanges (Fry \& Cheah, 2016; Brière, Oosterlinck, \& Szafarz, 2015) and the presence of price bubble in Bitcoin market (Cheung, Roca, \& Su, 2015; Cheah \& Fry, 2015) provide motivation for investigating the volatility spillover from Bitcoin market to the U.S. financial markets and foreign exchange pairs of six major trading currencies. Moreover, the detached behaviour of Bitcoin price formation from the economic fundamentals makes it a significant portfolio diversification instrument for conventional and alternative investment assets (Koutmos, 2018; Brière et al., 2015; Kristoufek, 2015). Urquhart and Zhang, (2018) proposed that Bitcoin is an effective hedge instrument for Euro, Swiss Franc and Great Britain Pound, whereas for Japanese Yen, Canadian dollar and Australian dollar it is an effective portfolio diversifier. The varying efficiency, arbitrage, hedging and portfolio diversification opportunities across Bitcoin exchanges denominated in different currencies provide motivation for empirically analysing whether Bitcoin currency denomination alter its portfolio diversification benefits.

In this paper, we investigate intra- and inter markets return spillovers for Bitcoin indices and foreign exchange pairs of the 6 major trading currencies (United States Dollar-USD, EURO, Japanese Yen-JPY, Great Britain Pound-GBP, Australian Dollar-AUD, and Canadian Dollar-CAD). The major trading currencies are selected 
based on their trading volume as trading volume is most likely to be affected by other financial and alternative investments assets. Moreover the selected markets possess the most mature and developed Bitcoin and foreign exchange markets. Along with this the selected Bitcoin markets has complete set of data for the entire analysis period. Due to increased integration of global financial markets the correlation among conventional investment opportunities has increased (Qarni \& Gulzar, 2019). The increased correlation has resulted in elimination of diversification benefits due to presence of contagion and spillover. With price and return behaviour unrelated to conventional investment assets, Bitcoin offers an attractive portfolio diversification tool for individual and institutional investors.

The basic idea that Bitcoin prices are inefficient, without any fundamental relationship to macroeconomic and financial variables, triggered another strand of studies examining the speculative nature of Bitcoin. Glaser, Zimmermann, Haferkorn, Weber, and Siering (2014) questioned the motivations behind the implementation of Bitcoin and highlight the resemblance of its exchange activities to pure speculative trading. Speculation and spillover are closely related phenomena as the former can give birth to the latter (Phillips \& Yu, 2011). Speculations generate an artificial price bubble which can burst anytime, transmitting fear among other financial markets' investors. The past experiences of financial crisis spillover and contagion among integrated financial markets due to speculative price bubbles has highlighted the importance of identification and curtailment of speculative price bubbles due to its devastating effects on other financial markets. This study provides novel evidence on three significant research questions based on the underlying research objectives and contributes to the existing literature. The first research objective analyses the degree of severity and patterns of intra- and inter- market return spillover in Bitcoin markets and foreign exchange pairs of six major trading currencies. The second objective is to analyse the degree of asymmetry in the return spillover between Bitcoin and foreign exchange pairs of six major trading currencies. The third research objective analyses the nature of connectedness at short and long frequencies for return spillover in the Bitcoin and foreign exchange pairs of six major trading currencies.

The findings of the study indicate low level of return spillover among the Bitcoin markets and the foreign exchange pairs dominated in six major trading currencies. Presence of asymmetric behaviour for return spillover among the Bitcoin markets and foreign exchange pairs dominated in six major trading currencies is also found. The findings also provide significant evidence regarding the occurrence of return spillover at short horizon, within four days of an event. The findings are useful for academicians, researchers, investors and policy makers. A clear understanding of the nature and dynamics of return spillover among the Bitcoin markets and foreign 
exchange pairs of six major trading currencies will equip investors and policy makers to better align their investment strategies and policies to counter the adverse conditions in either of the analysed markets. The findings of study regarding the portfolio diversification benefits of alternative currency trading in Bitcoin and foreign exchange markets provide new avenues to explore these aspects for other traditional and alternative investment assets. Currency of trade for Bitcoin and foreign exchange markets provides possibility of enhancing the diversification capabilities of these assets that provide valuable research gap for further analysis.

The next section provides a comprehensive literature review of the related research literature with an aim to uncover the research gap. Section 3 provides details of the methodologies employed for analysis. Section 4 provides descriptive of the data. The data description is provided in tabular and graphical representations. The results and discussions of the study are provided in Section 5. The last section provides the conclusion of the study, and also highlighting the major contribution, and implications of the study.

\section{Literature review}

Bitcoin provides an attractive investment alternative due to its high liquidity and ease of operation. Bitcoin is considered as a speculative investment option, due to the presence of high volatility like other financial markets. Speculation and spillover are closely related phenomena as the former can give birth to the latter (Phillips \& Yu, 2011; Gulzar, Kayani, Xiaofeng, Ayub, \& Rafique, 2019; Gulzar, Jan, \& Afzal, 2019). Speculations generate an artificial price bubble which can burst anytime, transmitting fear among other financial markets' investors. Cheah and Fry (2015) studied the high speculative nature of investors in the Bitcoin markets and defined Bitcoin markets as a price bubble accumulating market, prone to burst soon. Researchers have identified many of the characteristics of Bitcoins that makes it worthy to be considered as an alternative investment. By provision of alternative to paper money and a hedge instrument for investors in unstable inflationary economies (Richardson, 2014), Bitcoin markets are in a race to replicate the function of financial institutions (Kerner, 2014). Bitcoin provides portfolio diversification as an investment alternative like gold (Dyhberg, 2016b). With presence of unexplained nature of Bitcoin price movement by economic fundamentals, it provides a valuable means for portfolio diversification. Researcher has also warned for increased integration of Bitcoin market with other financial markets due to increasing acceptance of Bitcoin as a speculative investment instrument. The price efficiency mechanism in Bitcoin markets enhance the integration among the Bitcoin markets and foreign exchange markets, thus playing a role of inter-link between Bitcoin and other financial markets (Kurihare and Fukushima, 
2017). The effect of Bitcoin market on foreign exchange market can be explained it terms of its usage mechanism. If Bitcoin is used as a medium of exchange, the foreign exchange market is likely to be affected that will hamper the functioning of other financial markets as well.

With presence of extreme volatility like other financial markets, development in Bitcoin markets are considered to affect the functioning and investment level of other financial. Bitcoin market is characterized with speculative price bubble that we have witnessed during 2013 (Cheah and Fry, 2015). The financial markets price bubble and Bitcoin price bubble catches the attention of researchers to study the after math of such price bubbles (Qarni et al., 2019). Bitcoin provides hedging capabilities like gold, UK stock market and dollar, thus making it a significant portfolio diversification investment instrument (Dyhrberg, 2016a). Moreover, the author also found that Bitcoin market possesses high level of volatility as compared to gold, Foreign exchange and stock markets. Similarly, Bouri, Gupta, Tiwari, and Roubaud (2017) found that Bitcoin provide hedge against global uncertainty in the short run. On the other side, Bouri, Molnár, Azzi, Roubaud, and Hagfors (2017) found limited evidence for the integration and hedging capabilities of Bitcoin for world equity indices, bonds, oil, gold, general commodity index and U.S. Dollar index. Although researchers have investigated many aspects of the integration of Bitcoin markets with other financial markets, the published literature regarding the contagion and spillover among Bitcoin markets dominated in alternative currencies and the foreign exchange markets do not exist. As identified in the published research, the huge investors' attention gained by the Bitcoin markets has generated a speculative bubble in the market that can burst anytime (Cheah \& Fry, 2015). The crisis in Bitcoin markets can prove to be contagious, as published research has identified the existence of integration among the Bitcoin and other financial markets, especially foreign exchange markets. The published research has also identified that there exists price difference among the value of Bitcoin at difference Bitcoin exchanges denominated in different currencies. This is also an interesting avenue to investigate.

The non-ending debate and the presence of mixed evidence regarding the Bitcoin market's efficiency, speculative nature, and portfolio diversification capabilities, highlight the existence of research gap that need further analysis. The past research has also highlighted the presence of varying efficiency across Bitcoin exchanges denominated in alternative currencies. This finding has significant portfolio diversification benefits that need to be explored. Existing studies on spillover dynamics of Bitcoin denominated in alternative currencies is scare and most of the studies have focused their analysis on Bitcoin price data for a single currency, mainly U.S. dollar or weighted average price for Bitcoin. Similarly, for foreign exchange pairs, researchers 
have analysed foreign exchange of currencies against a single currency, mainly U.S. dollar or weighted average exchange rate against a group of currencies, thus limiting evidence on portfolio diversification benefits of alternative currency exchange rates. The present study covers the research gap on the unexplained nature of return spillover among the Bitcoin markets and Foreign exchange pairs denominated in six major trading currencies. The findings on the dynamics and nature of return spillover in Bitcoin markets and foreign exchange pairs of six major trading currencies will provide significant contribution to existing literature on spillover and contagion.

\section{Methodology}

Over the past numerous methodologies has been developed to investigate and measure spillover and contagion among conventional and alternative investment assets and markets. Derived from the Markowitz (1952) portfolio theory, correlation among conventional and alternative investment assets was considered as a major measure and transmission channel for spillover and contagion. The application of correlation as a measure of spillover and contagion was not free from criticism. The argument in favour of contagion effects of crisis was criticized by some researchers on the fact that these correlations were not adjusted for heteroskedasticity. If the correlations among the economies in the event of crisis are adjusted for the effect of heteroskedasticity, the result will show no increase in the correlation among the economies during the crisis event. Rather, it can be interpreted as the existence of interdependence among the economies (Basu, 2002; Bordo \& Murshid, 2001; Forbes \& Rigobon, 2002). In order to overcome the deficiencies of correlation model as a measure of spillover and contagion, Multivariate GARCH models were developed but were also not free of flaws. The estimates of BEKK-MGARCH model (Engle \& Kroner, 1995) do not provide a straight forward interpretation for the magnitude and direction of spillover and contagion due to its non-linear measurement properties. On other hand, Constant conditional correlation model (Bollerslev, 1990) provided a constant conditional correlation (CCC) measure for the entire sample period analyzed, which is in contradiction to the reality, as conditional correlation do not remain constant over time. Thus to capture the dynamic nature of conditional correlation among asset prices, a dynamic conditional correlation (DCC) Multivariate GARCH model was developed. The DCC MGARCH model provided a nondirectional measure of spillover and contagion for the entire set of economies, making it vague to identify the direction and magnitude of spillover among individual markets (Engle \& Sheppard, 2001). The criticism on the past research regarding contagion literature provided room for further analyses into the topic.

Over the time researchers have improved the methodological tools to provide 
insight into the existence of contagion among interlinked economies. Diebold and Yilmaz (2009) developed spillover index methodology to estimate the spillover among global equity markets but it was flawed as the results were dependent on the ordering of the variables. The dependence of results on the ordering of the variables was eliminated by Diebold and Yilmaz (2012), who provided measures to estimate the average static and dynamic spillover, along with directional and pairwise measures of spillover. It also allows estimation of time varying dynamics of spillover for conventional and alternative investment assets. Diebold and Yilmaz (2012) only provide aggregate measure of volatility spillover, the separate influence of positive and negative spillover could not be analysed by the methodology of Diebold and Yilmaz (2012). The idea of asymmetric spillover was introduced by Barunik, Kocenda and Vacha, (2015), by estimating separate $\mathrm{N}$-variable VAR models for positive and negative spillover. This methodology measured the isolated influence of positive volatility on positive and negative volatility on negative volatility only. However, it is significant to include the effect of negative volatility on positive volatility and vice versa. Barunik, Kocenda and Vacha (2017) extended the methodology by applying a single VAR model of 2N variables that includes both positive and negative volatilities for each variable. This model provided a more accurate measure for studying the influence of positive and negative volatility spillover in the financial and alternative assets' markets. Barunik et al., (2017) methodology accounted for influence of positive and negative volatility only at aggregate frequency level; however micro-analysis of spillover under time frequency domain is very important to understand the speed of spillover transmission among the integrated markets. Barunik and Krehlik (2016) provided a methodology to measure the estimates of spillover under time frequency domains. This methodology was further extended by Barunik and Krehlik (2018) to provide better estimates of frequency connected under time frequency domains.

With these attractive features, the joint application of spillover index (Diebold \& Yilmaz, 2012), spillover asymmetric measures (Barunik et al., 2017) and frequency connectedness (Barunik \& Krehlik, 2018) methodologies will provide a better insight into the nature and dynamics of return spillover among Bitcoin markets and foreign exchange pairs of six major trading currencies. These methodologies are the latest innovation and are most appropriate for analysing nature and dynamics of spillover among conventional and alternative investment assets.

To investigate the dynamics of return spillover among Bitcoin markets and foreign exchange markets of six major trading currencies the methodologies of spillover index (Diebold \& Yilmaz, 2012), spillover asymmetric measures (Barunik et al., 2017) and frequency connectedness (Barunik \& Krehlik, 2018) are applied. The spillover index analysis will provide valuable information on the nature of static and dynamic average 
return spillover among the analysed markets. The spillover asymmetric measures will uncover the influence of positive and negative volatilities of one market on the positive and negative volatilities of other markets. Whereas the frequency connected ness method will unveil the speed and horizon of spillover among the analysed markets.

\subsection{Spillover index}

To measure the spillover among the selected markets, we employed the methodology of Diebold and Yilmaz (2012), which eliminates the dependence of result on the ordering of the variables, by replacing the Cholesky factorization by KPPS (Koop, Pesaran, \& Potter, 1996; Pesaran \& Shin, 1998) variance decomposition. Instead of attempting to orthogonalize shocks, the generalized approach allows correlated shocks but accounts for them appropriately using the historical observed distribution of the errors. As the shocks to each variable are not orthogonalized, the sum of contributions to the variance of forecast error (that is, the row sum of the elements of variance decomposition table) is not necessarily equal to one.

An N-variable covariance stationary VAR (p), is defined as:

$x_{t}=\sum_{i=1}^{p} \theta_{t} x_{t-i}+\varepsilon_{t}$

Where represents the vector of returns, represents a parameter matrix and represent a vector for the disturbance with mean zero and constant variance.

The representation of the moving average is defined as:

$$
\begin{aligned}
& x_{t}=\sum_{i=0}^{\infty} B_{i} \varepsilon_{t-i} \\
& Q_{i}=\theta_{1} B_{i-1}+\theta_{2} B_{i-2}+\ldots .+\theta_{p} B_{i-p}
\end{aligned}
$$

Where $B_{i}$ is a $N \times N$ coefficient matrix obeying the recursion, $Q_{i}=\theta_{1} B_{i-1}+\theta_{2} B_{i-2}+\ldots .+\theta_{p} B_{i-p}$, with $B_{0}$ an $N \times N$ identity matrix and $B_{i}=0$ for $i<0$. The variance decompositions estimate the fraction of $\mathrm{H}$-step ahead error variance in forecasting $x_{i}$ that is due to shocks to $x_{j}$, such that $j \neq i$ for each $i$.

Let us define own variance shares to be the fraction of the $\mathrm{H}$-step ahead forecast error variances in forecasting $x_{i}$ due to shocks to $x_{i}$, for $i=1,2, \ldots . N$, and cross variance shares or spillover, to be the fractions of the $\mathrm{H}$-step ahead forecast error variances in forecasting xi due to shocks to $x_{j}$, for $i=1,2, \ldots . N$, and for $j=1,2, \ldots . N$, such that $j \neq i$.

Denoting the KPPS H-step ahead forecast error variance decomposition by $\phi_{i j}^{g}(H)$ $H=1,2, \ldots . N$, for we have

$$
\phi_{i j}^{g}(H)=\frac{\sigma_{i i}^{-1} \sum_{h=0}^{H-1}\left(e_{i}^{\prime} B_{h} \Psi e_{i}\right)^{2}}{\sum_{h=0}^{H-1}\left(e_{i}^{\prime} B_{h} \Psi B_{h}^{\prime} e_{i}\right)}
$$


Where $\Psi$ is variance matrix for error vectors $\varepsilon, \sigma_{i i}$ is the standard deviation of the error term for the ith equation $e_{i}$ and is the selection vector with one as the ith element and zero otherwise. As explained above, the sum of the elements of each row of the variance decomposition table is not equal to $1, \sum_{i=1}^{N} \phi_{j}^{g}(H) \neq 1$. To use the information in the variance decomposition matrix in the calculation of the spillover index, we normalize each entry of the variance decomposition matrix by the row sum. Alternatively, we can normalize the elements of the variance decomposition matrix with the column sum of these elements and compare the resulting total spillover index with the one obtained from the normalization with the row.

$$
\tilde{\phi}_{i j}^{g}(H)=\frac{\phi_{j}^{g}(H)}{\sum_{j=1}^{N} \phi_{j}^{g}(H)}
$$

Note that, by construction, $\sum_{j=1}^{N} \tilde{\phi}_{j i}^{g}(H)=1$, and $\sum_{i, j=1}^{N} \tilde{\phi}_{i j}^{g}(H)=N$.

Using the return spillover contributions from the KPPS variance decomposition, we can construct a total spillover index:

$$
S^{g}(H)=\frac{\sum_{\substack{i, j=1 \\ i \neq j}}^{N} \tilde{\phi}_{i j}^{g}(H)}{\sum_{i, j=1}^{N} \tilde{\phi}_{i j}^{g}(H)} \times 100=\frac{\sum_{\substack{i, j=j \\ i \neq j}}^{N} \tilde{\phi}_{i j}^{g}(H)}{N} \times 100
$$

This is the KPPS analogy of the Cholesky factor-based measure used in Diebold and Yilmaz (2012). The total spillover index measures the contribution of spillover to the total forecast error variance.

\subsection{Spillover asymmetry measure}

Spillover asymmetry measures (SAM) among the Bitcoin markets and the foreign exchange pairs dominated in six major trading currencies is examined by applying Barunik, Kočenda, and Vácha (2017) SAM methodology. To calculate the SAM, we decompose each return series into positive and negative series giving us $2 \mathrm{~N}$ variable model for returns and volatilities. SAM is measures as the difference between the positive and negative spillover for the "TO" and "FROM" spillover for returns and volatilities calculated by modified Diebold and Yilmaz (2012) model.

To calculate the SAM first we sum the corresponding row/column of the $2 N \times 2 N$ spillover matrix calculated by standard Diebold and Yilmaz (2012) method and exclude main diagonal of the $2 N \times 2 N$ spillover matrix and the two diagonals in the $N \times N$ sub-matrices on the lower left and the upper right of the $2 N \times 2 N$ main matrix. The methodology of SAM was introduced by Barunik, Kocenda, and Vacha (2015), in which separate spillover indices where calculated for positive and negative volatility 
before calculation of SAM. In contrast, Barunik, Kolenda, and Vácha (2017) extended their methodology by including positive and negative volatilities in single VAR model of $2 \mathrm{~N}$ variables.

The SAM with H-step ahead forecast at time $t, S A M_{2 N}^{H}$, is defined as the difference between return spillover due to positive and negative spillover. The SAM for directional TO spillover is defined as:

$$
S A M_{2 N}^{H}=S_{i,}^{g}(H)-S_{i+N}^{g},(H)
$$

Similarly, the SAM for directional FROM spillover can be calculated (For details see Barunik, Kočenda, and Vácha (2017))

\subsection{Frequency connectedness}

To analyse the frequency connectedness within Bitcoin and foreign exchange pairs dominated in six major trading currencies we employed the Barunik and Krehlik (2018) methodology. It applies spectral representations of variance decomposition method of Dew-Becker and Giglio (2016) to estimate frequency connectedness at short and long frequencies. Barunik and Krehlik (2016) introduced the notion of frequency connectedness relations in time frequency domain and were extended by Barunik and Krehlik (2018).

The spectral behaviour of series $X_{t}$ to decompose generalized impulse response function is shown as:

$$
S_{x}(w)=\sum_{h=0}^{\infty} E\left(X_{t} X_{t-h}\right) e^{-i h w}=\Psi\left(e^{i h w}\right)
$$

where $w$ denotes the frequency, $\infty$ denotes infinite horizon connectedness and $\Psi\left(e^{-i h w}\right)=\sum_{h=0}^{\infty} \Psi_{h} e^{-i h w}$ (Barunik \& Krehlik, 2016). The unconditional generalized forecast error variance decomposition (GFVED) on a particular frequency $w$ is calculated as:

$$
(\Theta(w))_{i, j}=\frac{\sigma_{j j}^{-1} \sum_{h=0}^{\infty}\left(\Psi\left(e^{-i h w}\right) \sum\right)_{i, j}^{2}}{\sum_{h=0}^{\infty}\left(\Psi\left(e^{-i h w}\right) \sum \Psi\left(e^{i h w}\right)\right)_{i, i}}
$$

Equation (9) can be standardized as:

$$
(\tilde{\Theta}(w))_{i, j}=\frac{(\Theta(w))_{i, j}}{\sum_{j=1}^{k}(\Theta(w))_{i, j}}
$$

Accumulative connectedness table over an arbitrary frequency band $d=(a ;, b)$ is expressed as:

$$
\left(\tilde{\Theta}_{d}\right)_{i, j}=\int_{a}^{b}(\tilde{\Theta}(w))_{i, j} d w
$$


The overall connectedness within a frequency band $d$ can be expressed as:

$$
C^{d}=\frac{\sum_{i, i, i, j}^{k}\left(\tilde{\Theta}_{d}\right)_{i, j}}{\sum_{i, j}\left(\tilde{\theta}_{d}\right)_{i, j}}=1-\frac{\sum_{i=1}^{k}\left(\tilde{\Theta}_{d}\right)_{i, j}}{\sum_{i, j}\left(\tilde{\theta}_{d}\right)_{i, j}}
$$

A value of $C^{d}$ close to unity indicates strong connections within the spectral band $d=(a ;, b)$. The within from connectedness measures the contribution of one market $(i \neq j)$ to another market $i$ on the spectral band $d$, which can be expressed as:

$$
C_{i \leftarrow}^{d}=\sum_{j=1, i \neq j}^{k}\left(\tilde{\Theta}_{d}\right)_{i, j}
$$

The within to connectedness measures the contribution to one market $(i \neq j)$ from another market $i$ on the spectral band $d$, which can be expressed as:

$$
C_{i \rightarrow}^{d}=\sum_{j=1, i \neq j}^{k}\left(\tilde{\Theta}_{d}\right)_{j, i}
$$
2012).

The total connectedness $C$ can be obtained by $S^{g}(H)=\sum_{d} \tilde{C}^{d}$ (Diebold and Yilmaz,

\section{Data and Summary statistics}

The data for each Bitcoin index and the foreign exchange pair consists of 1147 observations dated from September 17, 2014 to November 6, 2017. The sample period consists of both the tranquil and volatility period for the Bitcoin prices. The data for Bitcoin exchange ANX is used to represent Bitcoins denominated in JPY, GBP, AUD and CAD, whereas for USD and EURO, Bitfinex and Kraken exchange data are used. The Bitcoin markets and foreign exchange returns on non- synchronous holidays were calculated as zero. The zero returns on non-synchronous holidays mirror actual returns on non-trading days. The stock market returns were calculated as

$$
R_{t}=\ln \left(\frac{P_{t}}{P_{t-1}}\right) \times 100
$$

To apply the SAM model, we decompose each return series into positive and negative series, giving us a $2 \mathrm{~N}$ variable model for returns.

The descriptive statistics (Table 1) shows the presence of positive mean returns for the Bitcoin markets, whereas for the foreign exchange pairs' all mean returns are positive except for AUD:GBP and CAD:GBP. The standard deviation statistics indicate that the returns for the Bitcoin markets are more volatile than the foreign exchange returns. The statistics for the skewness, kurtosis and JB test indicate that none of the series is normally distributed. The ADF statistics are significant and shows that all series are stationary at level. 
Table 1: Descriptive Statistics: Returns

\begin{tabular}{|c|c|c|c|c|c|c|}
\hline \multicolumn{7}{|c|}{ Returns } \\
\hline & Mean & Std. Dev. & Skewness & Kurtosis & Jarque-Bera & ADF \\
\hline USD:BTC & 0.23 & 3.74 & -0.82 & 14.9 & 6884.53 & -35.85 \\
\hline EURO:BTC & 0.25 & 3.49 & -0.52 & 8.61 & 1556.38 & -33.68 \\
\hline JPY:BTC & 0.25 & 4.03 & -0.26 & 10.17 & 2465.29 & -38.4 \\
\hline GBP:BTC & 0.26 & 4.03 & -0.24 & 10 & 2351.48 & -37.94 \\
\hline AUD:BTC & 0.26 & 3.99 & -0.38 & 10.17 & 2482.18 & -38.2 \\
\hline CAD:BTC & 0.26 & 4.02 & -0.27 & 9.9 & 2285.26 & -38.16 \\
\hline EURO:USD & 0.01 & 0.51 & -0.22 & 6.85 & 718.29 & -35.1 \\
\hline JPY:USD & 0 & 0.54 & -0.58 & 9.83 & 2289.35 & -33.34 \\
\hline GBP:USD & 0.02 & 0.56 & 2.94 & 49.12 & 103217.9 & -35.93 \\
\hline AUD:USD & 0.01 & 0.55 & 0.17 & 5.16 & 228.91 & -35.99 \\
\hline CAD:USD & 0.01 & 0.46 & -0.11 & 5.19 & 231.65 & -34.69 \\
\hline JPY:EURO & 0 & 0.55 & -1.19 & 19.04 & 12561.29 & -34.12 \\
\hline GBP:EURO & 0.01 & 0.52 & 1.38 & 19.32 & 13076.48 & -33.25 \\
\hline AUD:EURO & 0 & 0.57 & 0.73 & 8.02 & 1305.28 & -33.82 \\
\hline CAD:EURO & 0 & 0.54 & 0.14 & 6.31 & 526.49 & -34.09 \\
\hline GBP:JPY & 0.01 & 0.73 & 4 & 70.55 & 220961.6 & -34.85 \\
\hline AUD:JPY & 0.01 & 0.68 & 1.1 & 12.13 & 4209.12 & -34.06 \\
\hline CAD:JPY & 0.01 & 0.66 & 0.67 & 10.49 & 2766 & -35.01 \\
\hline AUD:GBP & -0.01 & 0.6 & -0.98 & 16.36 & 8704.81 & -33.34 \\
\hline CAD:GBP & -0.01 & 0.56 & -1.42 & 20.42 & 14873.86 & -34.52 \\
\hline CAD:AUD & 0 & 0.44 & -0.2 & 5.08 & 214.91 & -34.35 \\
\hline Not: & 0.14 & \\
\hline
\end{tabular}

Note: USD, EURO, JPY, GBP, AUD and CAD is abbreviation for U.S. dollar, Euro, Japanese Yen, Great Britain Pound, Australian dollar, and Canadian dollar respectively. EURO: USD represents the foreign exchange pair for the Euro and U.S. dollar, and read as Euros per U.S. Dollars, same interpretation apply for other foreign exchange pairs. BTC-USD, BTC-EURO, BTC-JPY, BTC-GBP, BTC-AUD and BTC-CAD are the Bitcoins indices denominated in USD, EURO, JPY, GBP, AUD and CAD. The numeric values are rounded to 2 decimal places. The statistics are significant $1 \%$ level of significance.

\section{Empirical Results and discussion}

\subsection{Return spillover index (Table 2)}

The output of analysis is reported in the form of spillover tables for returns. The 
average returns spillover among Bitcoin markets and the foreign exchange pairs of 6 major trading currencies is $72.50 \%$ during the sampled period. The highest own market returns spillover contribution is shown by the CAD: AUD (42.4\%), with $58 \%$ contribution from others to its returns and 39\% contribution to others returns from CAD: AUD.

The GBP: BTC is the highest recipient of returns spillover from others $(80 \%)$, followed by JPY: BTC (79\%), AUD: BTC (79\%) and CAD: BTC (79\%). The highest return spillovers to GBP: BTC comes from CAD: BTC (18.6\%) and lowest return spillovers to GBP: BTC comes from JPY: USD, AUD: JPY, and CAD: AUD (0\% each). The return spillovers from the foreign exchange pairs of six major trading currencies to GBP: BTC range from $0 \%$ to $0.7 \%$, indicating minimal influence of foreign exchange markets on the returns of GBP: BTC.

The GBP: JPY is the highest transmitter of returns spillover to others (95\%), followed by AUD: JPY (94\%) and CAD: JPY (91\%). The highest returns spillover from GBP: JPY occurs to JPY: EURO (15.3\%), followed by GBP: EURO (13.7\%) and JPY: USD (13.5\%). The returns spillover from GBP: JPY to the Bitcoin markets range from $0 \%$ to $0.5 \%$, indicating less integration of foreign exchange markets with Bitcoin markets.

The findings indicate significant intra-Bitcoin markets returns spillover, with little influence from and to the foreign exchange pairs of major trading currencies. The GBP: BTC returns is most influenced by others (80\%) and CAD: AUD returns are least influenced by others (58\%).

The GBP: JPY influence the returns in others the most (95\%) and CAD: AUD least influence the returns in others (39\%). On average there exists a returns spillover of $72.5 \%$, with most of the spillover due to intra-Bitcoin markets' and intra-foreign exchange pairs' returns spillover. The evidence for low level of volatility spillover within the U.S. and financial markets is supported by the fact that the Bitcoin price movement is unrelated to the economic fundamentals (Dastgir, Demir, Downing, Gozgor, \& Lau, 2018; Ciaian et al., 2016). The detached behaviour of Bitcoin price movement makes it an important portfolio diversification instrument.

\subsection{Rolling window analysis - Returns spillover plot}

The time varying patterns of return spillovers are analysed by rolling window analysis with 200 days window and 10 steps forecast horizons. The rolling window analysis captures the cyclical patterns of the return spillover that is not captured by the static spillover index. Using a small rolling window length increases variance and result in large mean square forecast errors (Pesaran, \& Timmermann, 2002). 
The selection of window lengths without theoretical guidance is a common practice by forecasters (Molodtsova, \& Papell, 2009; Clark and West, 2007). The 200 days rolling window is selected to capture enough information so that we do not capture irrelevant information or loose important information. The robustness of the applied models are checked by changing the rolling window length and results are found to be robust, with low sensitivity to rolling window length.

The time varying return (Figure 1) spillover depict almost time varying patterns with decline in spillover during the $2^{\text {nd }}$ quarter of 2015 till the $3^{\text {rd }}$ quarter of 2015 due to stabilization of Euro-zone. The return spillover show a rising trend afterward till $4^{\text {th }}$ quarter of 2015 due to the Chinese stock market crash on June 12, 2015.

In the first quarter of 2016 the return spillover decline due to the end of Chinese stock market turbulence in February 2016. In June 2016 the returns spillover shows a sharp increase due to Brexit announcement on June 23, 2016, with gradual decrease afterward. In the $4^{\text {th }}$ quarter of 2016 the return spillover again increased due to uncertain in global financial market caused by Chinese reform initiative in August 2016. By the start of $20171^{\text {st }}$ quarter the return spillover have fallen sharply and depicts more stable behaviour.

As revealed from the static spillover analysis for the returns, most of return spillover occurs due to intra-Bitcoin markets and intra-foreign exchange pairs' returns spillover. Therefore, the varying patterns of returns spillover depicted in the rolling window analysis shows the patterns of intra-Bitcoin markets and intra- foreign exchange pairs returns spillovers.

\subsection{Spillover asymmetry measures}

The $2 \mathrm{~N}$ variables spillover indices for positive and negative return spillover among the Bitcoin and foreign exchange pairs denominated in six major trading currencies are depicted in table 3 ( $2 \mathrm{~N}$ variable Return spillover index). The findings of the analysis revealed that return $(73.8 \%)$ spillover among the Bitcoin and foreign exchange pairs dominated in six major trading currencies has increased due to incorporation of separate influence of positive and negative spillover. These findings clearly highlight the presence of asymmetry in the spillover among the selected markets.

The $2 \mathrm{~N}$ variables return spillover index (Table 3 ) indicates that positive returns for GBP:JPY pair received the highest return spillover (80.64) from others and transmitted the highest return spillover (98.31) to others. Whereas, positive returns for the CAD:AUD pair received the lowest return spillover (60.35) from other and transmitted the lowest return spillover (41.53) to other Bitcoin and foreign exchange pairs dominated in six major trading currencies. The highest own market return spillover 


\begin{tabular}{|c|c|c|c|c|c|c|c|c|c|c|c|c|c|c|}
\hline ડ.ә૫મО шод & 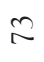 & $\stackrel{2}{2}$ & $\curvearrowright$ & 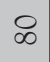 & 2 & $\curvearrowright$ & $\bullet$ & ชิ & $\approx$ & $\approx$ & $\bullet$ & ㅇ & $\infty$ & $N$ \\
\hline 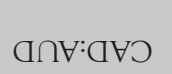 & 0 & 0 & 0 & 0 & $\rightleftarrows$ & $\ddot{0}$ & $\stackrel{\infty}{\circ}$ & $\stackrel{\infty}{\circ}$ & $\begin{array}{l}\circ \\
\dot{0}\end{array}$ & $\stackrel{0}{0}$ & $\sim$ & 0 & $\stackrel{\sim}{0}$ & $\begin{array}{l}\infty \\
\text { in }\end{array}$ \\
\hline 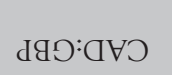 & 0 & 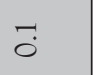 & $\overrightarrow{0}$ & $\dot{\circ}$ & $\overrightarrow{0}$ & 0 & $\sim$ & $\overrightarrow{0}$ & $\begin{array}{l}0 \\
\mathcal{I}\end{array}$ & $\stackrel{?}{\circ}$ & $\ddot{n}$ & $\stackrel{+}{\dot{-}}$ & $\stackrel{\curvearrowright}{\sim}$ & $\stackrel{+}{\sim}$ \\
\hline 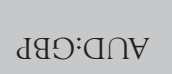 & 0 & 0 & $\overrightarrow{0}$ & $\dot{\leftarrow}$ & 0 & 0 & $\stackrel{+}{\circ}$ & $\stackrel{+}{0}$ & $\underset{\infty}{\sim}$ & $\underset{\infty}{+}$ & $\stackrel{n}{-}$ & $\stackrel{\eta}{-}$ & $\stackrel{?}{\sim}$ & $\stackrel{+}{\circ}$ \\
\hline 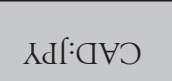 & 0 & 0 & $\stackrel{\sim}{\circ}$ & 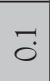 & $\overrightarrow{0}$ & $\overrightarrow{0}$ & $\stackrel{+}{\circ}$ & $\ddot{\sigma}$ & $\stackrel{\sim}{-}$ & $\stackrel{9}{-}$ & $\stackrel{0}{\exists}$ & $\stackrel{n}{=}$ & $\stackrel{m}{m}$ & $\stackrel{\sim}{m}$ \\
\hline 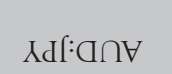 & 0 & 0 & $\dddot{0}$ & 0 & $\stackrel{\sim}{0}$ & 0 & $\ddot{0}$ & $\stackrel{\check{\Xi}}{=}$ & $\stackrel{\sim}{i}$ & $\simeq$ & $\ddot{n}$ & $\stackrel{+}{\circ}$ & 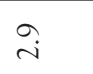 & $\tilde{0}$ \\
\hline xdઈ:dGつ & 0 & $\overrightarrow{0}$ & 0 & $\stackrel{n}{0}$ & $\stackrel{\sim}{0}$ & $\overrightarrow{0}$ & $\stackrel{n}{0}$ & $\stackrel{n}{n}$ & $\stackrel{\sim}{\ddot{n}}$ & $\begin{array}{l}\infty \\
0\end{array}$ & $\stackrel{0}{-}$ & in & $\ddot{\theta}$ & $\stackrel{\sim}{0}$ \\
\hline oษคヨ:৫৮ว & 0 & $\ddot{0}$ & $\widetilde{O}$ & $\ddot{0}$ & $\breve{0}$ & $\overrightarrow{0}$ & $\ddot{n}$ & $\stackrel{\infty}{\sim}$ & $\ddot{0}$ & $\tilde{0}$ & $\stackrel{n}{\infty}$ & $\ddot{\sim}$ & $\tilde{\sigma}$ & $\vec{g}$ \\
\hline 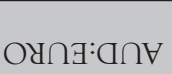 & 0 & $\ddot{0}$ & $\overrightarrow{0}$ & $\overrightarrow{0}$ & $\overrightarrow{0}$ & $\ddot{0}$ & 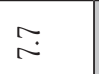 & - & $\stackrel{\sim}{0}$ & $\tilde{0}$ & $\stackrel{\vartheta}{i}$ & $\stackrel{+}{i}$ & $\stackrel{\sim}{n}$ & $\underset{\sim}{\sim}$ \\
\hline O४คヨ:dgり & 0 & 0 & 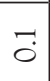 & $\stackrel{?}{0}$ & $\overrightarrow{0}$ & $\dddot{0}$ & $\stackrel{\infty}{i n}$ & $\stackrel{\sim}{m}$ & $\stackrel{a}{\infty}$ & $\stackrel{\sim}{0}$ & $\stackrel{\sim}{0}$ & $\stackrel{2}{0}$ & $\begin{array}{l}\stackrel{0}{\dot{m}} \\
\text {. }\end{array}$ & $\begin{array}{l}\stackrel{\sigma}{+}\end{array}$ \\
\hline O४คヨ:Хdl & 0 & $\stackrel{\sim}{0}$ & 0 & $\stackrel{\sim}{0}$ & $\overrightarrow{0}$ & $\overrightarrow{0}$ & $\infty$ & $\stackrel{+}{\circ}$ & $\stackrel{\sim}{\sim}$ & $\begin{array}{l}\infty \\
0 \\
0\end{array}$ & $\stackrel{\circ}{-}$ & ?̊n. & $\stackrel{?}{0}$ & $\vec{i}$ \\
\hline 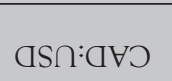 & 0 & $\ddot{0}$ & $\overrightarrow{0}$ & $\overrightarrow{0}$ & $\overrightarrow{0}$ & $\stackrel{\sim}{\circ}$ & $\stackrel{+}{+}$ & $\tilde{0}$ & $\begin{array}{l}\stackrel{0}{+} \\
\stackrel{+}{*}\end{array}$ & $\begin{array}{l}\infty \\
= \\
=\end{array}$ & $\begin{array}{l}\infty \\
m\end{array}$ & $\stackrel{+}{-}$ & $\tilde{0}$ & $\stackrel{+}{i}$ \\
\hline 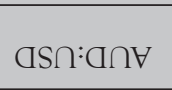 & 0 & $\ddot{0}$ & 0 & 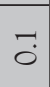 & $\stackrel{\sim}{0}$ & $\overrightarrow{0}$ & $\ddot{n}$ & $\stackrel{n}{\sim}$ & in & $\begin{array}{l}\dot{\sim} \\
\grave{\sim}\end{array}$ & $\tilde{n}$ & $\begin{array}{l}\infty \\
0\end{array}$ & $\stackrel{\sim}{0}$ & $\ddot{0}$ \\
\hline ๔Sへ:વЯつ & 0 & $\stackrel{m}{0}$ & $\widetilde{O}$ & $\tilde{0}$ & $\tilde{0}$ & $\stackrel{\sim}{0}$ & $\stackrel{+}{\sigma}$ & $\tilde{o}$ & $\underset{\sim}{\stackrel{\sim}{\sim}}$ & in & $i n$ & $\stackrel{n}{\sim}$ & $\overrightarrow{0}$ & $\breve{0}$ \\
\hline 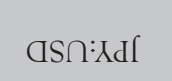 & 0 & $\ddot{0}$ & $\stackrel{\sim}{\circ}$ & 0 & $\overrightarrow{0}$ & 0 & $\stackrel{\sim}{6}$ & $\stackrel{\sim}{m}$ & $\tilde{c}$ & $\stackrel{+}{-}$ & $\stackrel{\circ}{\circ}$ & $\stackrel{\sim}{0}$ & $\ddot{n}$ & $\stackrel{\infty}{\circ}$ \\
\hline 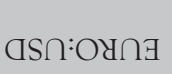 & 0 & $\stackrel{t}{\circ}$ & $\overrightarrow{0}$ & $\overrightarrow{0}$ & $\overrightarrow{0}$ & $\overrightarrow{0}$ & $\stackrel{+}{\dot{m}}$ & $\tilde{i n}$ & $\underset{\sim}{\stackrel{\sim}{\sim}}$ & $\stackrel{\leftrightarrow}{+}$ & $\stackrel{+}{+}$ & 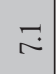 & $\stackrel{\sim}{n}$ & ํ. \\
\hline 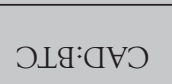 & $\begin{array}{l}0 \\
\mathcal{I}\end{array}$ & $\stackrel{m}{\sim}$ & $\curvearrowright$ & $\begin{array}{l}0 \\
\infty \\
0\end{array}$ & $\begin{array}{l}\infty \\
\infty \\
\infty\end{array}$ & $\begin{array}{l}0 \\
\dot{0}\end{array}$ & $\overrightarrow{0}$ & 0 & $\stackrel{+}{\circ}$ & 0 & $\stackrel{\sim}{O}$ & $\overrightarrow{0}$ & $\tilde{0}$ & 0 \\
\hline 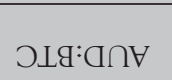 & $\begin{array}{l}0 \\
\stackrel{J}{U}\end{array}$ & $\ddot{n}$ & $\begin{array}{l}\stackrel{+}{0} \\
\infty\end{array}$ & $\begin{array}{l}n \\
\infty \\
0\end{array}$ & $\tilde{i}$ & $\begin{array}{l}\infty \\
\infty \\
\infty\end{array}$ & $\ddot{0}$ & 0 & $\dot{0}$ & $\tilde{0}$ & $\stackrel{\sim}{\circ}$ & $\overrightarrow{0}$ & $\stackrel{+}{\circ}$ & $\overrightarrow{0}$ \\
\hline วLg:વ9り & $\tilde{\beth}$ & $\begin{array}{l}0 \\
\end{array}$ & $\begin{array}{l}\stackrel{\rho}{\infty} \\
\infty\end{array}$ & $\overrightarrow{0}$ & 2 & 이 & $\stackrel{n}{0}$ & 0 & $\exists$ & 0 & $\overrightarrow{0}$ & $\tilde{0}$ & $\tilde{0}$ & 0 \\
\hline JLG:XdI & $\stackrel{\nabla}{\beth}$ & $\overrightarrow{0}$ & $\vec{\sim}$ & $\stackrel{\sim}{\infty}$ & $\stackrel{\sim}{\infty}$ & $\stackrel{\sim}{\infty}$ & $\ddot{0}$ & $\stackrel{\sim}{0}$ & $\tilde{0}$ & $\circ$ & 0 & 0 & $\tilde{0}$ & 0 \\
\hline วLg:О४คヨ & $\tilde{\sim}$ & $\stackrel{\stackrel{\sim}{\sim}}{\sim}$ & $\stackrel{n}{=}$ & $\stackrel{n}{=}$ & $\stackrel{n}{=}$ & $\stackrel{n}{=}$ & $\tilde{0}$ & $\overrightarrow{0}$ & $\dot{0}$ & $\overrightarrow{0}$ & $\widetilde{O}$ & $\stackrel{2}{0}$ & $\tilde{0}$ & $\overrightarrow{0}$ \\
\hline \multirow[t]{2}{*}{ วLg : đSก } & $\begin{array}{l}n \\
\tilde{v}\end{array}$ & $\begin{array}{l}\infty \\
\stackrel{\sim}{0} \\
\stackrel{\sim}{0}\end{array}$ & $\stackrel{\partial}{a}$ & $\stackrel{\alpha}{\alpha}$ & 으 & 으 & 0 & 0 & $\vec{\circ}$ & 0 & $\overrightarrow{0}$ & 0 & $\stackrel{\sim}{\circ}$ & 0 \\
\hline & 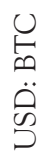 & 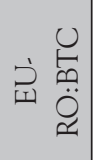 & 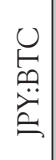 & $\begin{array}{l}0 \\
0 \\
\oplus \\
0 \\
0 \\
0\end{array}$ & 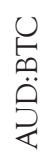 & $\begin{array}{c}u \\
\stackrel{\infty}{\ddot{\theta}} \\
\dot{\delta}\end{array}$ & 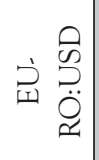 & 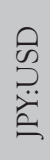 & $\begin{array}{l}0 \\
D \\
. \ddot{0} \\
0 \\
0\end{array}$ & 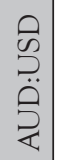 & 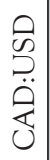 & 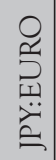 & 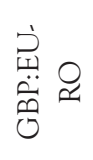 & 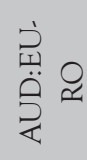 \\
\hline
\end{tabular}




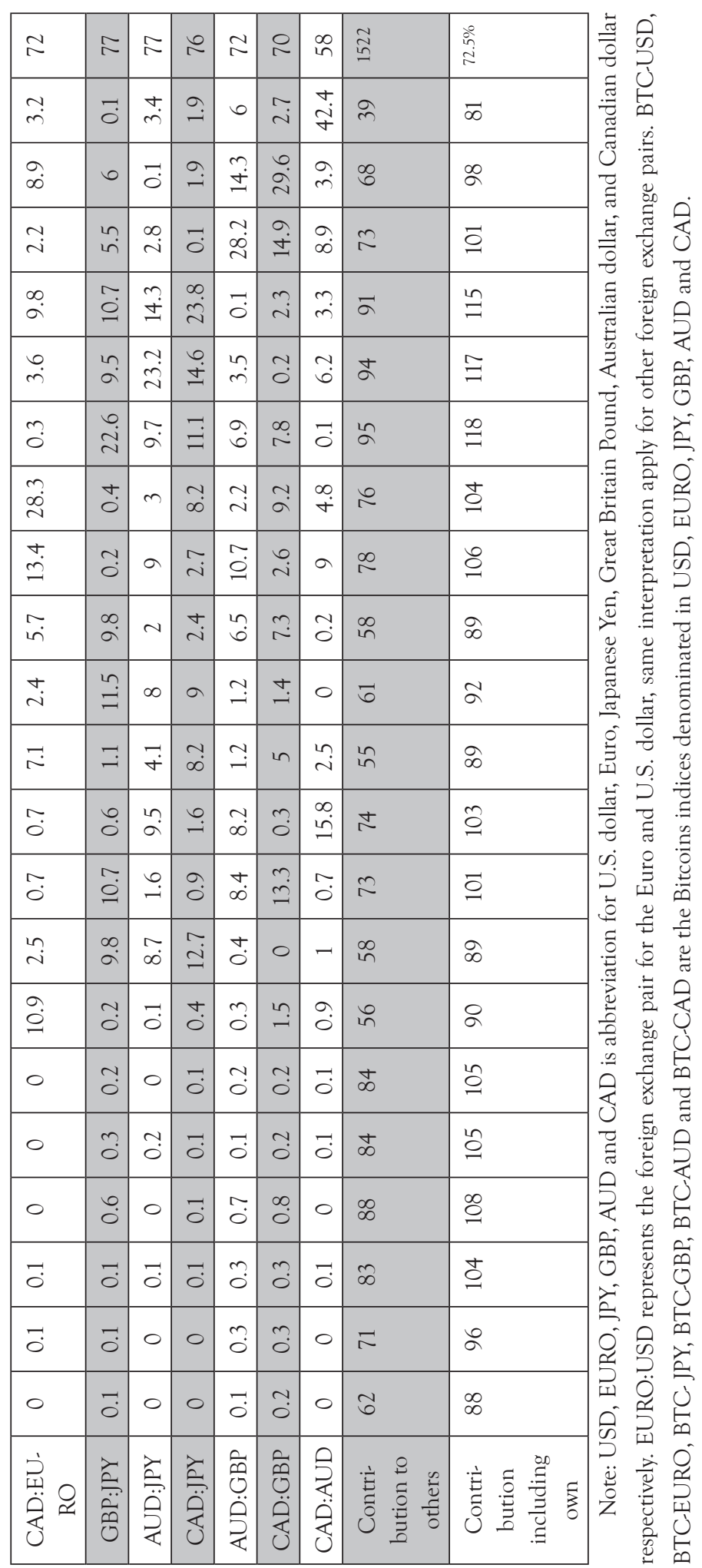




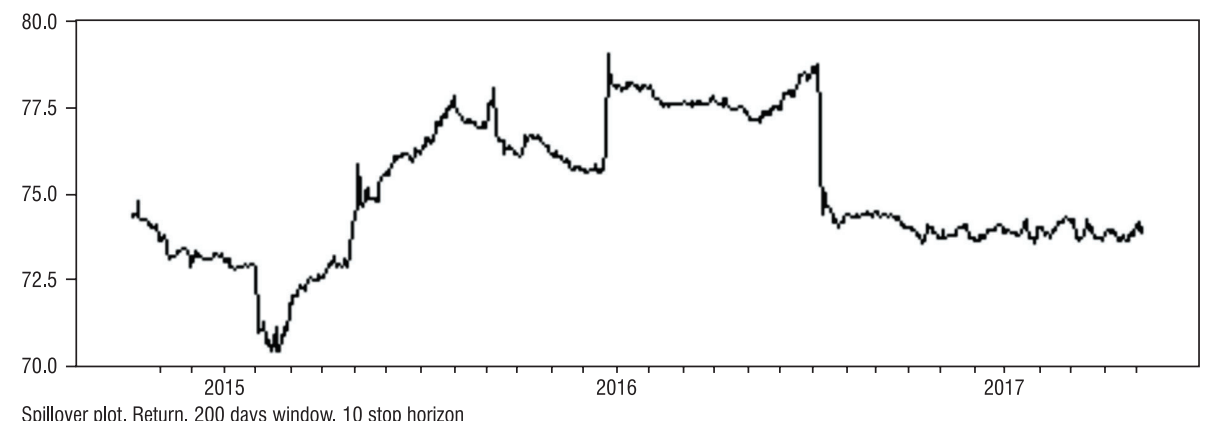

Note: The year identification marks indicate mid-year.

Figure 1: Returns Spillover Plot - Bitcoin Markets and Foreign Exchange Pairs of Six Major Trading Currencies

is depicted by positive returns in CAD:AUD pair (36.18). The lowest own market persistence in return spillover is depicted by positive returns in GBP:BTC (17.76).

The time varying nature of SAM among the Bitcoin markets and foreign exchange pairs dominated in six major trading currencies is depicted in figures 2 and 3 . The graphical analysis clearly reveals the presence of asymmetric return spillover among the Bitcoin markets and foreign exchange pairs of six major trading currencies.

The "FROM others SAM" depicted in figure 2 is estimated as the difference between positive and negative return spillover from others. The market for which "FROM others SAM" is positive indicates receipt of excess positive spillover and are considered as receiver of net positive spillover from others. For the markets for which "FROM others SAM" is negative indicates receipt of excess negative spillover and are considered as receiver of net negative spillover. In depth analysis of the "FROM others SAM" return spillover (Figure 2) reveals that EURO:BTC, CAD:BTC, EURO:USD, GBP:USD, AUD:USD, CAD:USD, GBP:EURO, AUD:EURO, GBP:JPY, AUD:JPY, and CAD:JPY received net positive return spillover and BTC:USD, EURO:BTC, GBP:BTC, AUD:BTC, JPY:USD, JPY:EURO, CAD:EURO, AUD:GBP, CAD:GBP, and CAD:AUD received net negative return spillover from other during the sampled period. The highest net positive spillover from other is received by GBP:EURO (3.76) and the lowest net positive spillover from others is received by JPY:BTC $(0.23)$. The highest net negative spillover is received by JPY:USD (-3.28) and the lowest net negative spillover is received by AUD:GBP (-0.05).

The "TO others SAM" depicted in figure 3 is estimated as the difference between positive and negative return spillover to others. The market for which "TO others SAM" is positive indicates transmission of excess positive spillover and are consid- 


\begin{tabular}{|c|c|c|c|c|c|c|c|c|c|c|c|c|c|c|c|c|c|}
\hline 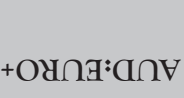 & $\stackrel{7}{\circ}$ & $\stackrel{\tilde{0}}{\circ}$ & $\stackrel{ }{\circ}$ & oे. & $\stackrel{\widetilde{N}}{\tilde{0}}$ & $\stackrel{m}{0}$ & $\begin{array}{l}+ \\
\infty \\
0 \\
0\end{array}$ & ָี & $\ddot{\circ}$ & $\begin{array}{l}\text { రై } \\
\text { o }\end{array}$ & $\begin{array}{r}\text { 于े } \\
\dot{m}\end{array}$ & $\begin{array}{l}\infty \\
\infty \\
i\end{array}$ & $\begin{array}{l}\hat{\sigma} \\
\dot{m}\end{array}$ & $\begin{array}{l}\infty \\
\stackrel{\sim}{\sim} \\
\tilde{v}\end{array}$ & 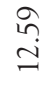 & $\stackrel{\Re}{+}$ & $\underset{\sim}{\stackrel{\Xi}{\sim}}$ \\
\hline 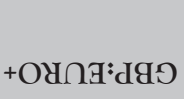 & $\exists$ & $\stackrel{8}{\circ}$ & $\begin{array}{l}\infty \\
\stackrel{0}{0}\end{array}$ & $\tilde{0}$ & 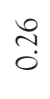 & $\stackrel{0}{0}$ & $\stackrel{7}{\circ}$ & $\tilde{o}$ & $\begin{array}{l}\circ \\
\infty \\
\infty\end{array}$ & $\stackrel{\varpi}{-}$ & $\stackrel{\text { gे }}{\dot{r}}$ & $\stackrel{\sim}{\rightarrow}$ & $\begin{array}{l}\stackrel{\sim}{\sim} \\
\underset{\sim}{\sim}\end{array}$ & $\begin{array}{l}\infty \\
\stackrel{\sim}{\sim}\end{array}$ & $\stackrel{0}{\stackrel{0}{*}}$ & $\tilde{\sigma}$ & $\underset{\sim}{\stackrel{+}{\sim}}$ \\
\hline +O\&กヨः Xdf & $\begin{array}{l}\stackrel{+}{\circ} \\
\stackrel{0}{ }\end{array}$ & $\stackrel{\circ}{\circ}$ & $\overrightarrow{0}$ & $\begin{array}{l}\text { Ln } \\
0 \\
0\end{array}$ & $\stackrel{\text { Oִ }}{0}$ & $\begin{array}{l}+ \\
0 \\
0\end{array}$ & $\begin{array}{l}\hat{b} \\
0 \\
0\end{array}$ & $a$ & $\hat{o}$ & $\stackrel{0}{\circ}$ & $\begin{array}{l}\vec{\sigma} \\
\stackrel{0}{0}\end{array}$ & $\begin{array}{l}n \\
\alpha \\
\infty \\
\sim\end{array}$ & $\stackrel{\tilde{\sigma}}{-}$ & $\stackrel{n}{\sim}$ & $\stackrel{+}{m}$ & $\stackrel{\Xi}{\rightarrow}$ & $\begin{array}{l}\infty \\
\infty \\
\infty \\
0\end{array}$ \\
\hline$+\mathrm{as} \Omega:$ aVD & $\frac{9}{0}$ & $\stackrel{\widetilde{\imath}}{0}$ & $\stackrel{\overbrace{}}{\tilde{0}}$ & $\tilde{\tilde{0}}$ & $\stackrel{+}{\tilde{0}}$ & $\stackrel{\infty}{\stackrel{\infty}{0}}$ & ळ̆ & $\stackrel{n}{-}$ & $\underset{\stackrel{\infty}{\sim}}{\stackrel{\infty}{m}}$ & $\frac{n}{a}$ & 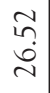 & $\overrightarrow{\tilde{o}}$ & $\stackrel{\Xi}{\leftrightarrows}$ & $\underset{\stackrel{\sim}{\sim}}{\stackrel{a}{2}}$ & $\vec{\vartheta}$ & セ̊ & $\tilde{m}$ \\
\hline$+\mathrm{QS} \Omega: \mathrm{d} \cap \mathrm{H}$ & $\underset{0}{\stackrel{7}{0}}$ & ઼ָ & $\stackrel{2}{0}$ & $\stackrel{\infty}{\stackrel{\infty}{0}}$ & $\stackrel{m}{0}$ & $\begin{array}{l}0 \\
0 \\
0\end{array}$ & $\begin{array}{l}\stackrel{\sigma}{ } \\
\stackrel{+}{ }\end{array}$ & $\vec{i}$ & $\stackrel{\dddot{q}}{\dot{r}}$ & $\begin{array}{l}\tilde{\sigma} \\
\tilde{\sim}\end{array}$ & $\begin{array}{l}\widetilde{\sigma} \\
\stackrel{0}{0}\end{array}$ & $\stackrel{m}{0}$ & $\stackrel{n}{\sim}$ & $\stackrel{\circ}{\circ}$ & $\vec{\sim}$ & $\stackrel{\sim}{\leftarrow}$ & $\stackrel{2}{\infty}$ \\
\hline +QSS:dGЭ & ڤ̊) & $\begin{array}{l}\stackrel{0}{0} \\
0\end{array}$ & ò & $\begin{array}{l}8 \\
0 \\
0\end{array}$ & $\stackrel{\widetilde{N}}{\stackrel{0}{0}}$ & $\stackrel{\infty}{\underset{0}{0}}$ & กี & $\stackrel{\curvearrowright}{\stackrel{0}{0}}$ & $\stackrel{\tilde{n}}{\vec{\sim}}$ & $\begin{array}{l}\widetilde{b} \\
\dot{r}\end{array}$ & $\stackrel{\Re}{\underset{r}{*}}$ & $\overline{\tilde{o}}$ & $\begin{array}{l} \pm \\
\stackrel{\sim}{a}\end{array}$ & $\begin{array}{l}\infty \\
\stackrel{0}{0} \\
0\end{array}$ & $\stackrel{\circ}{\circ}$ & $\begin{array}{l}\tilde{y} \\
\stackrel{0}{0}\end{array}$ & $\stackrel{\vec{n}}{\sim}$ \\
\hline$+\mathrm{CS} \cap$ & oे & $\begin{array}{l}\text { J } \\
\dot{0}\end{array}$ & $\begin{array}{l}\infty \\
0 \\
0\end{array}$ & $\stackrel{\tilde{o}}{0}$ & $\begin{array}{l}\overrightarrow{0} \\
\dot{0}\end{array}$ & $\begin{array}{l}\tilde{\sigma} \\
\text { Oे }\end{array}$ & $\vec{H}$ & $\begin{array}{l}\infty \\
\stackrel{n}{\sim} \\
\stackrel{\sim}{N}\end{array}$ & $\bar{\sigma}$ & $\stackrel{n}{n}$ & $\stackrel{\text { f }}{-}$ & 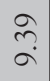 & $\tilde{0}$ & $\begin{array}{l}\infty \\
0 \\
0\end{array}$ & $\stackrel{\infty}{\stackrel{\infty}{0}}$ & 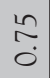 & $\begin{array}{l}3 \\
0 \\
0\end{array}$ \\
\hline 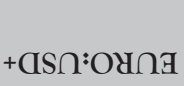 & $\overrightarrow{0}^{\circ}$ & $\frac{10}{0}$ & \& & $\stackrel{0}{\stackrel{0}{0}}$ & $\begin{array}{l}n \\
0 \\
0\end{array}$ & $\begin{array}{l}\text { In } \\
\text { Oे }\end{array}$ & $\begin{array}{l}0 \\
\infty \\
\sim\end{array}$ & $\begin{array}{l}\text { J } \\
\text { in }\end{array}$ & $\begin{array}{l}\curvearrowright \\
\dot{+}\end{array}$ & $\begin{array}{l}\stackrel{0}{ } \\
\sim \\
m\end{array}$ & $\begin{array}{l}n \\
n \\
n\end{array}$ & in. & $\stackrel{2}{0}$ & 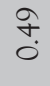 & مِ & $\begin{array}{l}\text { nn } \\
\infty \\
0\end{array}$ & $\stackrel{n}{0}$ \\
\hline +PLGः GVO & वे & $\hat{\alpha}$ & $\stackrel{\widetilde{\alpha}}{\stackrel{0}{0}}$ & $\begin{array}{l}\overline{\text { n}} \\
\text { o }\end{array}$ & 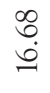 & $\vec{\infty}$ & $\begin{array}{l}\stackrel{8}{\circ} \\
\stackrel{0}{0}\end{array}$ & $\begin{array}{l}\hat{O} \\
\dot{0}\end{array}$ & $\stackrel{\infty}{\stackrel{0}{0}}$ & $=$ & $\stackrel{?}{\stackrel{0}{0}}$ & $\vec{\square}$ & $\stackrel{2}{?}$ & $\stackrel{\nabla}{\square}$ & $\underset{ָ}{\tilde{0}}$ & $\stackrel{\widetilde{\jmath}}{\text { ஸ̆ }}$ & $\frac{a}{0}$ \\
\hline +OLG: GกV & $\begin{array}{l}+ \\
\infty \\
\sigma\end{array}$ & $\stackrel{\infty}{\dddot{0}}$ & 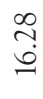 & $\begin{array}{l}\text { ర్ } \\
\stackrel{n}{n}\end{array}$ & $\stackrel{\overbrace{}}{\stackrel{0}{\infty}}$ & 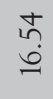 & $\stackrel{\circ}{\circ}$ & $\begin{array}{l}\tilde{O} \\
\text { Oे }\end{array}$ & 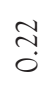 & $\stackrel{\widetilde{\jmath}}{0}$ & $\stackrel{n}{0}$ & $\stackrel{\sim}{\dddot{0}}$ & $\stackrel{+}{\stackrel{\sim}{0}}$ & $\vec{n}$ & $\stackrel{\widetilde{0}}{0}$ & $\stackrel{\text { ஸे }}{0}$ & $\stackrel{+}{\circ}$ \\
\hline +OLGःdGO & 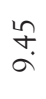 & $\begin{array}{l}\tilde{O} \\
\stackrel{0}{0}\end{array}$ & $\begin{array}{l}\bar{\infty} \\
\stackrel{0}{0}\end{array}$ & 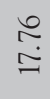 & oे & $\begin{array}{l}0 \\
\stackrel{0}{0} \\
0\end{array}$ & $\stackrel{\stackrel{J}{0}}{\circ}$ & $\stackrel{+}{\stackrel{J}{0}}$ & $\underset{0}{\approx}$ & $\stackrel{?}{\dddot{0}}$ & $\tilde{\tilde{c}}$ & $\stackrel{\infty}{\underset{0}{0}}$ & $\underset{\mathfrak{0}}{\stackrel{n}{0}}$ & $\begin{array}{l}\text { oे } \\
\text { o. }\end{array}$ & $\stackrel{\sim}{\dddot{0}}$ & 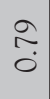 & $\stackrel{\overbrace{}}{\grave{0}}$ \\
\hline +OLG:Xdl & ๗ે. & $\begin{array}{l}\hat{\sigma} \\
\text { aे }\end{array}$ & $\begin{array}{l}\tilde{\circ} \\
\stackrel{0}{0}\end{array}$ & $\begin{array}{l}\text { ठ } \\
\stackrel{0}{0}\end{array}$ & $\stackrel{\circ}{\sim}$ & $\begin{array}{l}\stackrel{\infty}{+} \\
\stackrel{0}{0}\end{array}$ & $\begin{array}{l}\infty \\
\stackrel{0}{\circ} \\
\stackrel{0}{0}\end{array}$ & $\stackrel{ \pm}{\stackrel{ \pm}{0}}$ & $\stackrel{\sim}{\dddot{0}}$ & $\begin{array}{l}n \\
\stackrel{2}{0} \\
0\end{array}$ & $\exists$ & $\stackrel{\dddot{m}}{\mathrm{r}}$ & $\stackrel{\infty}{\stackrel{\infty}{0}}$ & $\underset{0}{\exists}$ & $\ddot{0}$ & $\stackrel{\circ}{\circ}$ & $\tilde{O}$ \\
\hline +OLG:O\&ดA & 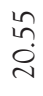 & $\begin{array}{l}\stackrel{\circ}{\vec{\lambda}} \\
\text { }\end{array}$ & $\begin{array}{l}\underset{+}{+} \\
\infty\end{array}$ & $\begin{array}{l}\stackrel{0}{2} \\
\infty \\
\infty\end{array}$ & $\begin{array}{l}\vec{b} \\
\infty\end{array}$ & $\begin{array}{l}\tilde{N} \\
\infty\end{array}$ & $\stackrel{\overrightarrow{0}}{0}$ & గo. & $\stackrel{0}{\stackrel{0}{0}}$ & $\stackrel{7}{0}$ & $\stackrel{m}{\circ}$ & $\stackrel{\infty}{\underset{0}{0}}$ & $\stackrel{\square}{\leftrightarrows}$ & $\ddot{0}$ & $\overrightarrow{0}$ & $\stackrel{0}{\stackrel{0}{0}}$ & $\stackrel{\square}{\leftrightarrows}$ \\
\hline \multirow[t]{2}{*}{ +OLg : AS $\Omega$} & 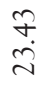 & ঐે & $\underset{\stackrel{\overbrace{}}{\circ}}{\stackrel{8}{2}}$ & $\stackrel{+}{\sim}$ & 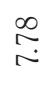 & in & $\stackrel{\text { Oै }}{0}$ & $\overrightarrow{0}$ & $\tilde{O}$ & 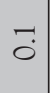 & $\begin{array}{l}\stackrel{n}{O} \\
0\end{array}$ & $\stackrel{\sim}{3}$ & $\stackrel{7}{\circ}$ & $\begin{array}{l}\stackrel{0}{0} \\
\stackrel{0}{0}\end{array}$ & $\stackrel{n}{0}$ & $\stackrel{\sim}{\dddot{0}}$ & $\underset{\Xi}{\stackrel{\Xi}{\sigma}}$ \\
\hline & $\begin{array}{l}+ \\
\bullet \\
\bullet \\
\ddot{\theta} \\
\stackrel{0}{D}\end{array}$ & 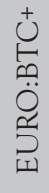 & 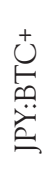 & $\begin{array}{l}+ \\
\sqcup \\
\stackrel{0}{0} \\
\ddot{0} \\
0\end{array}$ & 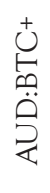 & 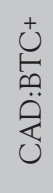 & 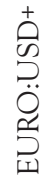 & 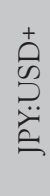 & 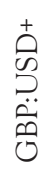 & 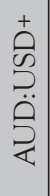 & 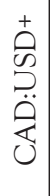 & 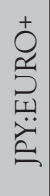 & 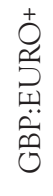 & 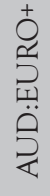 & 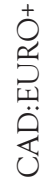 & $\begin{array}{l}+ \\
\stackrel{+}{0} \\
\ddot{0} \\
0\end{array}$ & 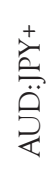 \\
\hline
\end{tabular}




\begin{tabular}{|c|c|c|c|c|c|c|c|c|c|c|c|c|c|c|c|c|c|c|c|c|c|}
\hline $\begin{array}{l}\infty \\
\infty \\
i\end{array}$ & $\begin{array}{c}\text { ஸे } \\
\stackrel{0}{0}\end{array}$ & $\begin{array}{l}\infty \\
\stackrel{\infty}{\sim}\end{array}$ & $\underset{\square}{\Xi}$ & \begin{tabular}{l}
\multirow{O}{0}{} \\
$\stackrel{0}{0}$
\end{tabular} & $\stackrel{2}{2}$ & б̊ & $\begin{array}{l}\infty \\
0 \\
0\end{array}$ & $\begin{array}{l}n \\
0 \\
0\end{array}$ & $\begin{array}{l}+ \\
0 \\
0\end{array}$ & $\begin{array}{l}8 \\
0 \\
0\end{array}$ & $\stackrel{+}{\leftarrow}$ & 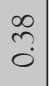 & بْ & サே. & 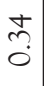 & $\hat{\sigma}$ & $\begin{array}{l}\vec{\sim} \\
\vec{\sim}\end{array}$ & $\stackrel{\stackrel{\sim}{\rightleftarrows}}{-}$ & $\begin{array}{l}\text { กี } \\
0\end{array}$ & ذे & $\underset{\widetilde{\sigma}}{\tilde{O}}$ \\
\hline $\begin{array}{l}\vec{F} \\
\dot{m}\end{array}$ & $\stackrel{7}{0}$ & $\begin{array}{l}\tilde{n} \\
\tilde{0}\end{array}$ & $\begin{array}{l}\hat{\jmath} \\
\dot{0}\end{array}$ & $\hat{O}$ & $\begin{array}{l} \pm \\
0 \\
0\end{array}$ & $\tilde{o}$ & $\begin{array}{l}\infty \\
0 \\
0\end{array}$ & ஜ̊. & $\begin{array}{l}\tilde{0} \\
0\end{array}$ & $\begin{array}{c}\tilde{\sigma} \\
i\end{array}$ & $\begin{array}{l}n \\
\tilde{n} \\
\dot{n}\end{array}$ & \begin{tabular}{l}
\multirow{J}{*}{} \\
$\dot{0}$
\end{tabular} & 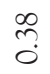 & $\frac{2 n}{0}$ & $\stackrel{+}{\rightarrow}$ & $\stackrel{\text { }}{\sim}$ & 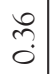 & $\begin{array}{l}+ \\
\dot{0}\end{array}$ & $\hat{\sigma}$ & $\stackrel{\Xi}{ \pm}$ & సै \\
\hline$\underset{ت}{\leftrightarrows}$ & $\hat{\sigma}$ & $\exists$ & 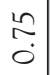 & $\stackrel{\text { Lo }}{\circ}$ & 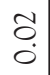 & $\frac{m}{0}$ & ¿̊. & Oे. & \begin{tabular}{l}
$\infty$ \\
\hdashline \\
0
\end{tabular} & $\underset{6}{\tilde{\sigma}}$ & $\begin{array}{l}\stackrel{2}{2} \\
\stackrel{0}{0}\end{array}$ & $\sqrt{\mathfrak{n}}$ & ـ & $\stackrel{\infty}{\stackrel{\infty}{+}}$ & $\begin{array}{l}\text { oे } \\
\text {. }\end{array}$ & $\begin{array}{l}\vec{\sim} \\
\tilde{0}\end{array}$ & $\tilde{\tilde{o}}$ & $\stackrel{0}{\circ}$ & $\begin{array}{l}\widetilde{\sigma} \\
\sigma\end{array}$ & 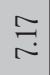 & $\begin{array}{l}\vec{\infty} \\
0 \\
0\end{array}$ \\
\hline$\vec{G}$ & $\begin{array}{l}0 \\
\sim \\
\sim\end{array}$ & 긍 & $\underset{\sim}{ \pm}$ & $\begin{array}{l}\infty \\
\stackrel{0}{0} \\
0\end{array}$ & $\overrightarrow{0}$ & $\begin{array}{l}\text { Jे } \\
\stackrel{0}{\circ}\end{array}$ & $\begin{array}{l}\circ \\
\stackrel{0}{0}\end{array}$ & $\stackrel{m}{0}$ & $\stackrel{7}{\dddot{0}}$ & $\stackrel{\sim}{\dot{0}}$ & $\underset{\sim}{\stackrel{J}{0}}$ & $\begin{array}{l}\infty \\
\infty \\
0 \\
0\end{array}$ & 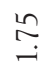 & $\begin{array}{l}8 \\
\dot{r} \\
\end{array}$ & $\underset{-}{\stackrel{ }{-}}$ & $\stackrel{\sim}{\stackrel{H}{0}}$ & 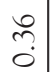 & $\stackrel{n}{\hat{0}}$ & $\tilde{\tilde{0}}$ & $\tilde{\sigma}$ & $\stackrel{9}{\rightleftarrows}$ \\
\hline $\begin{array}{l}\text { oे } \\
\text { i }\end{array}$ & 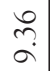 & 守 & $\stackrel{\text { }}{-}$ & $\begin{array}{l}2 \\
\stackrel{2}{0} \\
0\end{array}$ & $\begin{array}{l}n \\
0 \\
0\end{array}$ & $\begin{array}{l}\text { ठ } \\
\text { : }\end{array}$ & $\begin{array}{l}\circ \\
\circ \\
0\end{array}$ & $\stackrel{3}{0}$ & $\begin{array}{l}\circ \\
\circ \\
0\end{array}$ & $\begin{array}{l}\dot{m} \\
\dot{0}\end{array}$ & $\underset{\tilde{~}}{\tilde{0}}$ & $\dot{0}$ & $\begin{array}{l}\tilde{2} \\
\underset{\sim}{0}\end{array}$ & $\sqrt{\tilde{n}}$ & $\underset{\exists}{\Im}$ & $\stackrel{\sim}{+}$ & $\stackrel{\Re}{-}$ & $\stackrel{\sim}{\dddot{0}}$ & \begin{tabular}{l}
$\infty$ \\
\multirow{0}{0}{}
\end{tabular} & 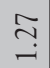 & $\overrightarrow{0}$ \\
\hline$\frac{\overrightarrow{\vec{i}}}{\mathrm{i}}$ & $\tilde{\tilde{o}}$ & $\exists$ & 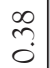 & $\stackrel{n}{0}$ & $\underset{0}{ت}$ & $\begin{array}{l}\infty \\
\stackrel{0}{\circ} \\
\stackrel{0}{2}\end{array}$ & $\stackrel{9}{\circ}$ & $\stackrel{m}{\circ}$ & $\begin{array}{l}0 \\
0 \\
0\end{array}$ & $\stackrel{m}{=}$ & $\underset{0}{0}$ & $\stackrel{+}{\stackrel{5}{-}}$ & $\begin{array}{l}\tilde{\sigma} \\
\dot{0}\end{array}$ & $\begin{array}{l}0 \\
+ \\
0\end{array}$ & $\vec{n}$ in & $\begin{array}{l}\dot{5} \\
\tilde{0}\end{array}$ & $\begin{array}{l}\stackrel{q}{+} \\
\dot{0}\end{array}$ & $\begin{array}{l}2 n \\
\infty \\
0 \\
0\end{array}$ & $\begin{array}{l}\stackrel{n}{\simeq} \\
0\end{array}$ & $\frac{\nabla}{0}$ & $\stackrel{\text { O̊ }}{0}$ \\
\hline 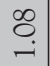 & $\begin{array}{l}8 \\
\stackrel{0}{0} \\
0\end{array}$ & $\tilde{n}$ & $\hat{\tilde{n}}$ & $\underset{0}{\stackrel{7}{0}}$ & $\stackrel{9}{0}$ & $\stackrel{?}{\stackrel{0}{0}}$ & $\begin{array}{l}8 \\
0 \\
0\end{array}$ & $\begin{array}{l}\infty \\
\stackrel{0}{0} \\
\stackrel{0}{0}\end{array}$ & $\begin{array}{l} \pm \\
0 \\
0\end{array}$ & $\begin{array}{c}\tilde{0} \\
0 \\
0\end{array}$ & $\underset{\sim}{\approx}$ & $\underset{\dot{0}}{ \pm}$ & ڤे. & $\exists$ & స్ర & $\frac{O}{\sim}$ & $\stackrel{\infty}{=}$ & $\underset{\sim}{\sim}$ & $\hat{\alpha}$ & $\stackrel{+}{\stackrel{5}{\sim}}$ & $\hat{0}$ \\
\hline 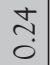 & $\stackrel{\sim}{\dddot{0}}$ & $\stackrel{7}{3}$ & $\stackrel{\widetilde{\jmath}}{\tilde{0}}$ & $\begin{array}{l}8 \\
\dot{0}\end{array}$ & 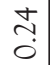 & $\stackrel{7}{0}$ & $\stackrel{\sim}{0}$ & $\tilde{0}$ & \begin{tabular}{c}
0 \\
\hdashline \\
0
\end{tabular} & ले & $\underset{0}{\tilde{0}}$ & $\begin{array}{l}\infty \\
\stackrel{2}{0}\end{array}$ & $\begin{array}{l}\tilde{n} \\
\dot{0}\end{array}$ & $\begin{array}{l}\infty \\
\stackrel{n}{0}\end{array}$ & in & Әे & $\frac{m}{6}$ & $\begin{array}{l}\widetilde{\alpha} \\
\infty\end{array}$ & $\underset{\varpi}{\stackrel{ \pm}{0}}$ & $\begin{array}{l}0 \\
+ \\
0\end{array}$ & $\begin{array}{c}\vec{\infty} \\
\dot{0}\end{array}$ \\
\hline$\tilde{\tilde{0}}$ & $\begin{array}{l}8 \\
0 \\
0\end{array}$ & $\frac{\infty}{3}$ & $\begin{array}{l}\widetilde{1} \\
0\end{array}$ & $\stackrel{n}{n}$ & $\begin{array}{l}\stackrel{n}{\simeq} \\
-\end{array}$ & . & $\stackrel{n}{n}$ & 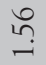 & $\underset{-}{\mathbb{b}}$ & $\stackrel{m}{0}$ & $\overrightarrow{\widetilde{\sigma}}$ & $\begin{array}{l}\text { பே. } \\
0\end{array}$ & $\hat{O}$ & ธo & $\stackrel{\overbrace{}}{0}$ & $\begin{array}{l}8 \\
0 \\
0\end{array}$ & $\begin{array}{c}\overrightarrow{0} \\
0\end{array}$ & $\hat{o}$ & $\begin{array}{l}\widetilde{O} \\
\stackrel{0}{0}\end{array}$ & oे & $\begin{array}{l}\infty \\
0 \\
0\end{array}$ \\
\hline సั. & $\frac{n}{0}$ & $\frac{7}{\circ}$ & ธல & $\begin{array}{l}\text { ?̊ } \\
\end{array}$ & రి & $\stackrel{f}{乛}$ & $\stackrel{\sim}{\underset{H}{*}}$ & 诒 & $\tilde{\sim}$ & $\stackrel{\sim}{\dddot{\imath}}$ & $\tilde{n}$ & $\stackrel{2}{2}$ & $\stackrel{7}{\circ}$ & ஜํ. & $\tilde{n}$ & ¿ & ? & రิ & $\hat{o}$ & $\stackrel{0}{=}$ & เి \\
\hline$\stackrel{\infty}{\stackrel{\infty}{0}}$ & 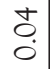 & ְै. & $\hat{O}$ & 志 & $\stackrel{+}{-}$ & $\stackrel{\vec{\sigma}}{.}$ & 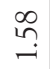 & in & $\underset{-}{\mathbb{G}}$ & $\underset{0}{\overparen{0}}$ & $\stackrel{+}{\tilde{0}}$ & $\stackrel{\sim}{\dddot{0}}$ & $\begin{array}{l}2 \\
0 \\
0\end{array}$ & $\begin{array}{l} \pm \\
0 \\
0\end{array}$ & $\begin{array}{l}\text { oे } \\
\text { : }\end{array}$ & $\begin{array}{l} \pm \\
0 \\
0\end{array}$ & $\begin{array}{l} \pm \\
0 \\
0\end{array}$ & $\begin{array}{l}\text { Oै } \\
0\end{array}$ & $\overrightarrow{0}$ & $\ddot{\circ}$ & $\begin{array}{l}n \\
0 \\
0\end{array}$ \\
\hline $\begin{array}{l}\tilde{O} \\
0\end{array}$ & $\begin{array}{l}\tilde{O} \\
0\end{array}$ & $\exists$ & $\begin{array}{l}\stackrel{+}{0} \\
\stackrel{0}{ }\end{array}$ & $\underset{\sim}{\stackrel{\tau}{-}}$ & $\stackrel{m}{\infty}$ & & กี & in & 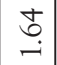 & $\frac{n}{30}$ & $\begin{array}{l}\dot{J} \\
0 \\
\end{array}$ & $\begin{array}{l}\tilde{0} \\
0\end{array}$ & $\begin{array}{l}\text { பே } \\
\stackrel{0}{\circ}\end{array}$ & $\begin{array}{l} \pm \\
0 \\
0\end{array}$ & $\begin{array}{l}\infty \\
\stackrel{0}{0} \\
\stackrel{0}{0}\end{array}$ & $\stackrel{m}{0}$ & $\begin{array}{l}0 \\
0 \\
0\end{array}$ & $\begin{array}{l}\text { ठே } \\
\stackrel{0}{0}\end{array}$ & $\stackrel{\sim}{\rightrightarrows}$ & oे & $\begin{array}{l}8 \\
\circ \\
0\end{array}$ \\
\hline$\stackrel{0}{\stackrel{0}{0}}$ & $\underset{\square}{ \pm}$ & $\stackrel{\Xi}{ \pm}$ & $\begin{array}{l}8 \\
: \\
\circ\end{array}$ & $\begin{array}{l}\underset{J}{U} \\
\text { i }\end{array}$ & $\begin{array}{l}\hat{b} \\
\text { i }\end{array}$ & กิ & & ?ִ & $\underset{\sim}{\stackrel{N}{*}}$ & ठे. & $\underset{0}{7}$ & $\stackrel{8}{8}$ & ธo. & $\begin{array}{l}\text { to } \\
0\end{array}$ & $\tilde{0}$ & $\frac{2}{8}$ & $\begin{array}{l}+ \\
0 \\
0\end{array}$ & బo & \begin{tabular}{l}
$\infty$ \\
\hdashline \\
0
\end{tabular} & oे & $\stackrel{0}{\circ}$ \\
\hline$\stackrel{m}{0}$ & $\underset{0}{\circ}$ & $\stackrel{\infty}{\circ}$ & $\tilde{O}$ & $\stackrel{+}{\sim}$ & $\stackrel{\widetilde{\sim}}{\sim}$ & $\underset{-}{\stackrel{\sim}{\sim}}$ & $\stackrel{\Re}{\rightarrow}$ & $\stackrel{+}{m}$ & $\underset{\sim}{\sim}$ & $\stackrel{2}{0}$ & $\underset{0}{\breve{0}}$ & $\begin{array}{l}\stackrel{+}{0} \\
\stackrel{0}{ }\end{array}$ & $\hat{O}$ & $\begin{array}{l}\tilde{O} \\
0\end{array}$ & $\stackrel{10}{\circ}$ & $\begin{array}{l}8 \\
0 \\
0\end{array}$ & $\begin{array}{l}0 \\
0 \\
0\end{array}$ & ठே. & $\begin{array}{l}8 \\
0 \\
0\end{array}$ & $\overrightarrow{0}$ & $\stackrel{\infty}{\infty}$ \\
\hline 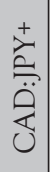 & 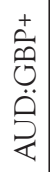 & $\begin{array}{l}+ \\
+ \\
0 \\
0 \\
\dot{0} \\
\text { ¿ }\end{array}$ & 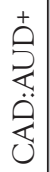 & 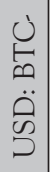 & 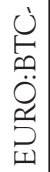 & 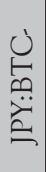 & $\begin{array}{l}\dot{U} \\
\stackrel{+}{\circ} \\
\stackrel{0}{0} \\
0\end{array}$ & 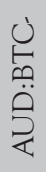 & 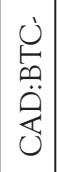 & 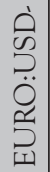 & 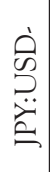 & $\begin{array}{l}\dot{0} \\
\text { Dे } \\
\ddot{0} \\
0 \\
0\end{array}$ & $\begin{array}{l}\dot{\rho} \\
\stackrel{\rho}{2} \\
\ddot{\theta} \\
\stackrel{2}{2}\end{array}$ & \begin{tabular}{l}
$\dot{\theta}$ \\
\multicolumn{2}{c}{} \\
$\ddot{\theta}$ \\
$\dot{c}$
\end{tabular} & 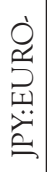 & 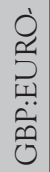 & 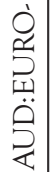 & 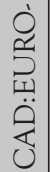 & 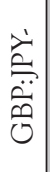 & 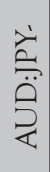 & 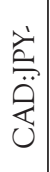 \\
\hline
\end{tabular}




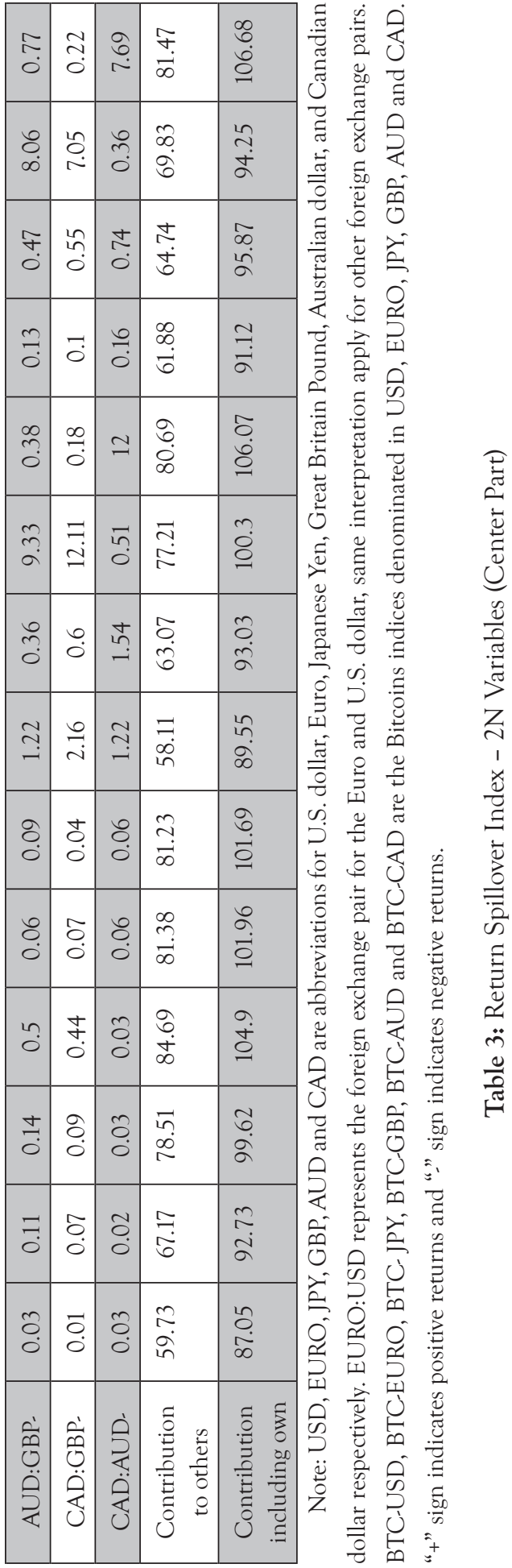

\begin{tabular}{|c|c|c|c|c|c|c|}
\hline - AS $\cap: X d I$ & 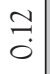 & $\begin{array}{l}+ \\
O\end{array}$ & in & $\stackrel{\check{J}}{\stackrel{\Delta}{0}}$ & $\stackrel{\text { I }}{\stackrel{0}{0}}$ & $\begin{array}{l}\stackrel{0}{0} \\
0\end{array}$ \\
\hline 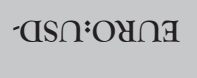 & \begin{tabular}{l}
$\circ$ \\
\hdashline \\
$\dot{0}$
\end{tabular} & ஜ̊ & Oे. & $\hat{O}$ & $\stackrel{n}{2}$ & ro. \\
\hline PLG:AVO & $\begin{array}{l}n \\
0 \\
ن\end{array}$ & $\stackrel{+}{\sim}$ & $\stackrel{\circ}{\sim}$ & $\stackrel{n}{\tilde{\sim}}$ & $\begin{array}{c}\infty \\
\sim \\
\sim\end{array}$ & $\tilde{\sim}$ \\
\hline OLG:AกV & 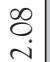 & $\stackrel{n}{\tilde{N}}$ & $\tilde{n}$ & $\begin{array}{l}\stackrel{\sim}{\sim} \\
\sim\end{array}$ & $\underset{\sim}{\sim} \underset{\sim}{\sim}$ & $\stackrel{⿱ 亠 凶}{\sim}$ \\
\hline -OLG:dG9 & $\begin{array}{l}n \\
0 \\
\sim \\
\sim\end{array}$ & $\stackrel{\circ}{\sim}$ & $\stackrel{n}{\sim}$ & ָे & 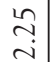 & 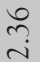 \\
\hline OLGःXdI & $\begin{array}{l}\infty \\
\stackrel{\infty}{-}\end{array}$ & $\underset{\sim}{\stackrel{\infty}{\sim}}$ & $\underset{\sim}{\sim}$ & ָे & $\begin{array}{l}\stackrel{0}{\sim} \\
\underset{\sim}{\sim}\end{array}$ & $\begin{array}{l}\text { o } \\
\stackrel{i}{i}\end{array}$ \\
\hline วLGःO\&กA & $\underset{m}{\tilde{m}}$ & $\stackrel{\circ}{\dot{m}}$ & $\check{-}$ & నె & 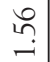 & 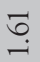 \\
\hline OLG : GS & $\begin{array}{l}\infty \\
\infty \\
\dot{r}\end{array}$ & $\begin{array}{l}\infty \\
\text { di }\end{array}$ & $\underset{+}{\stackrel{f}{+}}$ & กี & $\stackrel{\infty}{\underset{\sim}{\sim}}$ & $\stackrel{+}{\sim}$ \\
\hline 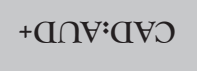 & $\begin{array}{l}\infty \\
0 \\
0 \\
0\end{array}$ & $\stackrel{\mathscr{m}}{0}$ & oे & $\begin{array}{l}8 \\
0 \\
0\end{array}$ & $\ddot{0}$ & $\begin{array}{l}\infty \\
\stackrel{0}{0} \\
\dot{0}\end{array}$ \\
\hline +dGЭः đVכ & $\frac{10}{0}$ & 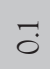 & $\overrightarrow{0}$ & $\begin{array}{l}n \\
\stackrel{n}{0} \\
0\end{array}$ & oे. & $\stackrel{ \pm}{ \pm}$ \\
\hline +dG९:વกV & $\stackrel{\sim}{\circ}$ & $\stackrel{\infty}{\dddot{0}}$ & $\exists$ & oे. & $\stackrel{2}{\circ}$ & $\exists$ \\
\hline$+X d[$ aVD & $\underset{0}{\overparen{1}}$ & $\stackrel{ \pm}{\leftrightarrows}$ & $\stackrel{?}{0}$ & \begin{tabular}{l}
0 \\
\multirow{2}{0}{}
\end{tabular} & $\stackrel{n}{\stackrel{n}{0}}$ & ڤे. \\
\hline$+X d[\because \mathrm{C} \cap \forall$ & $\stackrel{ \pm}{ \pm}$ & $\stackrel{2 n}{0}$ & $\stackrel{\text { oे }}{\circ}$ & $\overrightarrow{\tilde{o}}$ & $\stackrel{n}{\stackrel{n}{0}}$ & Iี \\
\hline +XdIःdg9 & $\overrightarrow{0}$ & $\stackrel{0}{0}$ & О̊. & $\stackrel{2}{\stackrel{2}{0}}$ & $\stackrel{t}{\circ}$ & 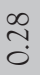 \\
\hline 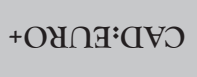 & $\stackrel{m}{0}$ & $\begin{array}{l}\infty \\
\stackrel{0}{0}\end{array}$ & $\stackrel{m}{\circ}$ & oे. & $\stackrel{\sim}{0}$ & $\frac{\partial}{0}$ \\
\hline
\end{tabular}




\begin{tabular}{|c|c|c|c|c|c|c|c|c|c|c|c|c|c|c|c|c|c|c|c|c|c|}
\hline$\stackrel{\infty}{\dddot{0}}$ & $\overrightarrow{\mathrm{i}}$ & 告 & $\stackrel{m}{0}$ & $\stackrel{\overbrace{}}{0}$ & $\underset{0}{\stackrel{N}{0}}$ & $\tilde{m}$ & $\exists$ & $\underset{ن}{+}$ & $\stackrel{\tilde{n}}{\sim}$ & $\underset{⿱}{\stackrel{J}{\sim}}$ & $\begin{array}{l}\tilde{b} \\
\stackrel{0}{0}\end{array}$ & $\stackrel{+}{\circ}$ & $\stackrel{n}{6}$ & ọ & $\begin{array}{l}\infty \\
0 \\
0\end{array}$ & $\stackrel{7}{0}$ & $\stackrel{m}{0}$ & $\stackrel{2}{0}$ & $\stackrel{\sim}{\dddot{0}}$ & $\stackrel{7}{\circ}$ & $\underset{\text { in }}{\bar{F}}$ \\
\hline $\begin{array}{l}\text { I } \\
\text { i }\end{array}$ & $\stackrel{\infty}{\circ}$ & $\hat{o}$ & $\stackrel{\infty}{+}$ & $\underset{0}{\overparen{0}}$ & ठे. & $\begin{array}{l}\vec{\infty} \\
\dot{m}\end{array}$ & $\underset{+}{\check{\sigma}}$ & $\begin{array}{l}\overbrace{}^{\circ} \\
\infty\end{array}$ & $\begin{array}{l}0 \\
0 \\
0\end{array}$ & $\underset{+}{\tilde{\sigma}}$ & $\underset{0}{\stackrel{N}{0}}$ & 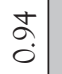 & 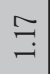 & $\stackrel{\infty}{-}$ & $\stackrel{2}{\circ}$ & 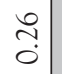 & $\stackrel{n}{2} \stackrel{0}{0}$ & $\stackrel{n}{\circ}$ & $\hat{O}$ & Ő & $\frac{n}{2}$ \\
\hline 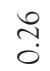 & $\stackrel{?}{0}$ & $\underset{\sim}{\stackrel{0}{0}}$ & $\stackrel{n}{\circ}$ & $\stackrel{\sim}{0}$ & $\begin{array}{l}\stackrel{0}{\circ} \\
0\end{array}$ & $\stackrel{\vec{\jmath}}{\circ}$ & $\begin{array}{l}\widetilde{O} \\
0\end{array}$ & ○े & $\underset{⿱}{\stackrel{t}{0}}$ & $\begin{array}{l}\tilde{O} \\
0\end{array}$ & $\begin{array}{l}\infty \\
0 \\
0 \\
0\end{array}$ & $\begin{array}{l}\dot{J} \\
0 \\
\end{array}$ & $\begin{array}{l}\infty \\
0 \\
0 \\
0\end{array}$ & $\frac{2 n}{0}$ & $\begin{array}{l}\tilde{O} \\
0\end{array}$ & $\begin{array}{l}+ \\
\infty \\
0 \\
0\end{array}$ & \begin{tabular}{l}
$\infty$ \\
\multirow{\sigma}{\sigma}{}
\end{tabular} & $\vec{\sigma}$ & బิ & $\stackrel{2}{\circ}$ & ப் \\
\hline خे & $\stackrel{\simeq}{0}$ & $\stackrel{\widetilde{N}}{0}$ & $\exists$ & $\stackrel{\sim}{0}$ & $\stackrel{⿱ 亠 士 冖}{0}$ & $\stackrel{\widetilde{T}}{0}$ & ठ․ & $\begin{array}{l}\tilde{O} \\
\stackrel{0}{0}\end{array}$ & $\stackrel{\widetilde{T}}{0}$ & $\begin{array}{l}2 \\
\stackrel{2}{0} \\
0\end{array}$ & ธิ & $\begin{array}{l}\infty \\
0 \\
0\end{array}$ & $\underset{0}{\stackrel{7}{0}}$ & $\stackrel{ \pm}{\square}$ & $\stackrel{\sim}{0}$ & $\stackrel{0}{0}$ & \begin{tabular}{l}
5 \\
\multirow{2}{*}{} \\
0
\end{tabular} & $\begin{array}{l}\hat{\sigma} \\
\doteq \\
=\end{array}$ & $\vec{\sigma}$ & $\begin{array}{l}\tilde{0} \\
0 \\
0\end{array}$ & பே. \\
\hline$\stackrel{3}{0}$ & $\stackrel{ \pm}{\square}$ & $\tilde{n}$ & Oै & $\begin{array}{l}\infty \\
\stackrel{0}{\circ}\end{array}$ & $\frac{10}{0}$ & $\overrightarrow{\tilde{0}}$ & $\begin{array}{l}8 \\
\stackrel{0}{0}\end{array}$ & $\begin{array}{l}20 \\
0 \\
0\end{array}$ & $\overrightarrow{\tilde{0}}$ & $\begin{array}{l}n \\
0 \\
0 \\
0\end{array}$ & ठ̊. & $\stackrel{\widetilde{N}}{\tilde{0}}$ & ๗ิ & $\exists$ & 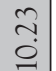 & $\stackrel{\infty}{\rightrightarrows} \underset{\exists}{=}$ & ఏे & $\begin{array}{l}\mathscr{2} \\
\infty \\
\infty\end{array}$ & $\begin{array}{l}\stackrel{\text { Dे }}{=} \\
=\end{array}$ & $\stackrel{\sim}{\stackrel{ }{~}}$ & $\tilde{0}$ \\
\hline$\stackrel{\overrightarrow{0}}{0}$ & $\stackrel{\infty}{\underset{\sim}{*}}$ & $\stackrel{\sim}{\circ}$ & $\begin{array}{l}\text { Oै } \\
\text { Oे }\end{array}$ & ک̊ & $\underset{0}{\tilde{0}}$ & $\vec{\sim}$ & $\begin{array}{l}\infty \\
\stackrel{0}{0} \\
0\end{array}$ & $\begin{array}{l}\tilde{O} \\
0\end{array}$ & 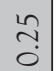 & $\frac{n}{0}$ & $\vec{\sim}$ & $\stackrel{\sim}{0}$ & $\stackrel{0}{0}$ & $\stackrel{m}{0}$ & $\begin{array}{l}\infty \\
\infty \\
\alpha\end{array}$ & 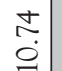 & $\stackrel{\sim}{2}$ & 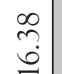 & $\stackrel{2}{6}$ & $\begin{array}{l}\infty \\
n \\
0 \\
0\end{array}$ & $\begin{array}{l}\text { பे } \\
\stackrel{0}{\circ}\end{array}$ \\
\hline 守 & $\stackrel{\infty}{\dddot{0}}$ & $\stackrel{?}{0}$ & $\begin{array}{l}\widetilde{O} \\
0\end{array}$ & $\ddot{0}$ & $\stackrel{\text { సี }}{0}$ & $\tilde{\tilde{0}}$ & $\stackrel{0}{\dddot{0}}$ & $\stackrel{\infty}{\circ}$ & \begin{tabular}{l}
0 \\
\multirow{1}{*}{}
\end{tabular} & $\begin{array}{l}8 \\
0 \\
0\end{array}$ & oे & $\stackrel{7}{\circ}$ & $\stackrel{\sim}{0}$ & $\overrightarrow{0}$ & ڤి. & $\begin{array}{l}i n \\
i n \\
\end{array}$ & 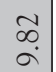 & $\begin{array}{l}\infty \\
a\end{array}$ & $\begin{array}{l}\text { ஸे } \\
\text { aे }\end{array}$ & $\begin{array}{l}+ \\
\infty \\
\circ\end{array}$ & $\begin{array}{l}\stackrel{0}{2} \\
\text { ?े }\end{array}$ \\
\hline ठे. & ঃ. & $\ddot{0}$ & $\begin{array}{l}\tilde{O} \\
0\end{array}$ & $\tilde{\circ}$ & 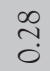 & $\stackrel{2}{\stackrel{2}{0}}$ & $\begin{array}{l}\text { Lo } \\
0 \\
0\end{array}$ & О̊. & $\begin{array}{l}0 \\
0 \\
0\end{array}$ & $\begin{array}{l}n \\
0 \\
0 \\
0\end{array}$ & ธิ & $\stackrel{n}{\circ}$ & $\stackrel{\vec{\sim}}{0}$ & $\underset{0}{ \pm}$ & \begin{tabular}{l}
\multirow{f}{*}{} \\
$\stackrel{2}{\sim}$
\end{tabular} & $\begin{array}{l}\vec{\imath} \\
\infty\end{array}$ & $\underset{\infty}{\stackrel{\sim}{\infty}}$ & $\frac{a}{\infty}$ & $\tilde{\infty}$ & $\infty_{\infty}^{\infty}$ & $\stackrel{\circ}{\stackrel{0}{0}}$ \\
\hline$\stackrel{\tilde{丶}}{0}$ & $\stackrel{0}{0}$ & $\underset{\tilde{0}}{\tilde{0}}$ & $\stackrel{\Xi}{\Xi}$ & $\stackrel{\infty}{\stackrel{\infty}{\rightarrow}}$ & $\stackrel{+}{\circ}$ & $\stackrel{\stackrel{m}{+}}{\dot{0}}$ & $\begin{array}{l}\overline{0} \\
\dot{0}\end{array}$ & กี & $\underset{0}{ \pm}$ & $\begin{array}{l}\infty \\
\\
0\end{array}$ & مִ & $\underset{0}{0}$ & $\hat{\tilde{n}}$ & \begin{tabular}{l}
$\infty$ \\
\hdashline \\
0
\end{tabular} & $\ddot{0}$ & $\stackrel{n}{0}$ & бo & $\begin{array}{l}\dot{0} \\
\dot{0}\end{array}$ & $\stackrel{\circ}{\circ}$ & oे & $\underset{-}{F}$ \\
\hline$\stackrel{\tilde{b}}{0}$ & กै? & $\begin{array}{l}+ \\
\infty \\
0 \\
0\end{array}$ & $\stackrel{\simeq}{\beth}$ & oे & $\tilde{\hat{o}}$ & సે & $\stackrel{0}{\stackrel{\sim}{i}}$ & $\tilde{\vartheta}$ & $\stackrel{\infty}{\dddot{0}}$ & $\underset{0}{\tilde{0}}$ & $\tilde{\sim}$ & $\begin{array}{l}\stackrel{\vartheta}{0} \\
\stackrel{0}{0}\end{array}$ & $\begin{array}{l}\underset{\sim}{J} \\
\text { స. }\end{array}$ & $\begin{array}{l}\infty \\
\stackrel{\sim}{n} \\
\end{array}$ & $\begin{array}{l}\text { Ln } \\
0 \\
0\end{array}$ & $\stackrel{n}{0}$ & $\stackrel{0}{0}$ & $\stackrel{9}{0}$ & రิ & $\stackrel{\circ}{\circ}$ & $\stackrel{\overbrace{}}{\leftrightarrows}$ \\
\hline$\stackrel{乛}{*}$ & $\hat{o}$ & $\tilde{\hat{0}}$ & $\begin{array}{l}\text { Dం } \\
\infty\end{array}$ & $\begin{array}{l}\infty \\
\infty \\
i \\
ن\end{array}$ & छे & Oे & $\stackrel{n}{a}$ & $\stackrel{\circ}{\sim}$ & $\begin{array}{l}\infty \\
\stackrel{0}{0}\end{array}$ & 广 & $\stackrel{?}{\rightleftarrows}$ & 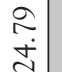 & $\begin{array}{l}\tilde{O} \\
\mathcal{U}\end{array}$ & $\stackrel{\overrightarrow{-}}{.}$ & $\hat{O}$ & $\begin{array}{l}+ \\
\\
\end{array}$ & ஓे & $\exists$ & ठ․ & $\stackrel{\widetilde{O}}{0}$ & 호 \\
\hline 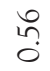 & $\stackrel{n}{n}$ & $\stackrel{\infty}{\sim} \underset{\sim}{\sim}$ & 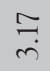 & $\underset{+}{\sim}$ & $\stackrel{ \pm}{\leftrightarrows}$ & $\begin{array}{l}\stackrel{+}{\circ} \\
\stackrel{+}{ }\end{array}$ & $\vec{m}$ & 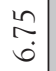 & $a$ & $\frac{n}{\stackrel{n}{c}}$ & $\begin{array}{l}20 \\
0 \\
\sigma\end{array}$ & $\stackrel{n}{n}$ & $\stackrel{\sim}{\stackrel{\sim}{\sim}}$ & $\begin{array}{l}\overrightarrow{0} \\
\dot{v}\end{array}$ & $\begin{array}{l}\widetilde{O} \\
0 \\
0\end{array}$ & $\stackrel{2}{0}$ & $\stackrel{7}{0}$ & $\begin{array}{l}0 \\
\circ \\
0\end{array}$ & $\stackrel{\text { Ln }}{\circ}$ & $\hat{O}$ & $\begin{array}{l}\infty \\
\infty \\
\circ\end{array}$ \\
\hline$\tilde{\check{0}}$ & ڤ̆ & $\stackrel{\widehat{~}}{\text {. }}$ & $\stackrel{\infty}{\stackrel{0}{0}}$ & $\begin{array}{l}\text { े } \\
\text { in }\end{array}$ & ڤn & $\sigma$ & $a$ & $\begin{array}{l}\stackrel{\infty}{\sim} \\
\ddot{m}\end{array}$ & $\begin{array}{c}\tilde{O} \\
\infty \\
\infty\end{array}$ & $\begin{array}{l}\tilde{n} \\
\infty \\
\infty\end{array}$ & $\begin{array}{l}\infty \\
\\
\simeq\end{array}$ & $\stackrel{0}{\stackrel{0}{+}}$ & $\underset{\sim}{\stackrel{\sim}{-}}$ & $\underset{\dot{0}}{\stackrel{ \pm}{0}}$ & ธ్ & مَ & $\stackrel{m}{0}$ & $\hat{O}$ & $\hat{O}$ & Oै & $\tilde{\tilde{n}}$ \\
\hline$\stackrel{\vec{I}}{\stackrel{-}{*}}$ & ڤ̆ & $\underset{\beth}{\stackrel{\Xi}{\Xi}}$ & $\underset{-}{\approx}$ & $\vec{\sim}$ & 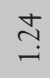 & $\stackrel{\tilde{n}}{=}$ & กี้ & กี้ & 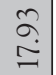 & $\underset{\infty}{\stackrel{+}{d}}$ & $\begin{array}{l}\stackrel{\infty}{\sim} \\
\stackrel{n}{\alpha}\end{array}$ & $\exists$ & ?ొ & $\stackrel{0}{\dddot{0}}$ & $\begin{array}{l}\text { Oे } \\
\stackrel{0}{0}\end{array}$ & $\begin{array}{l}\infty \\
\stackrel{0}{0}\end{array}$ & $\begin{array}{l}\text { t. } \\
\end{array}$ & $\overrightarrow{0}$ & $\begin{array}{l}\infty \\
\stackrel{0}{0}\end{array}$ & \& & $\stackrel{m}{0}$ \\
\hline$\stackrel{\widetilde{\sigma}}{-}$ & $\stackrel{\text { ñ }}{\stackrel{0}{0}}$ & $\tilde{0}$ & $\underset{\sim}{\sim}$ & $\underset{\sigma}{\sigma}$ & जे & $\stackrel{n}{+}$ & $\stackrel{゚}{\stackrel{2}{\Xi}}$ & $\stackrel{\tilde{\jmath}}{\check{\tau}}$ & 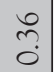 & $\underset{\infty}{\infty}$ & 둘 & $\begin{array}{l}\stackrel{0}{1} \\
\stackrel{m}{*}\end{array}$ & తి & $\vec{\infty}$ & $\begin{array}{l}\overline{0} \\
\dot{0}\end{array}$ & $\begin{array}{l}\infty \\
0 \\
0\end{array}$ & $\underset{7}{\stackrel{7}{0}}$ & $\begin{array}{l}8 \\
0 \\
0\end{array}$ & ڤno & $\begin{array}{l}+ \\
\\
\end{array}$ & $\stackrel{n}{f}$ \\
\hline
\end{tabular}




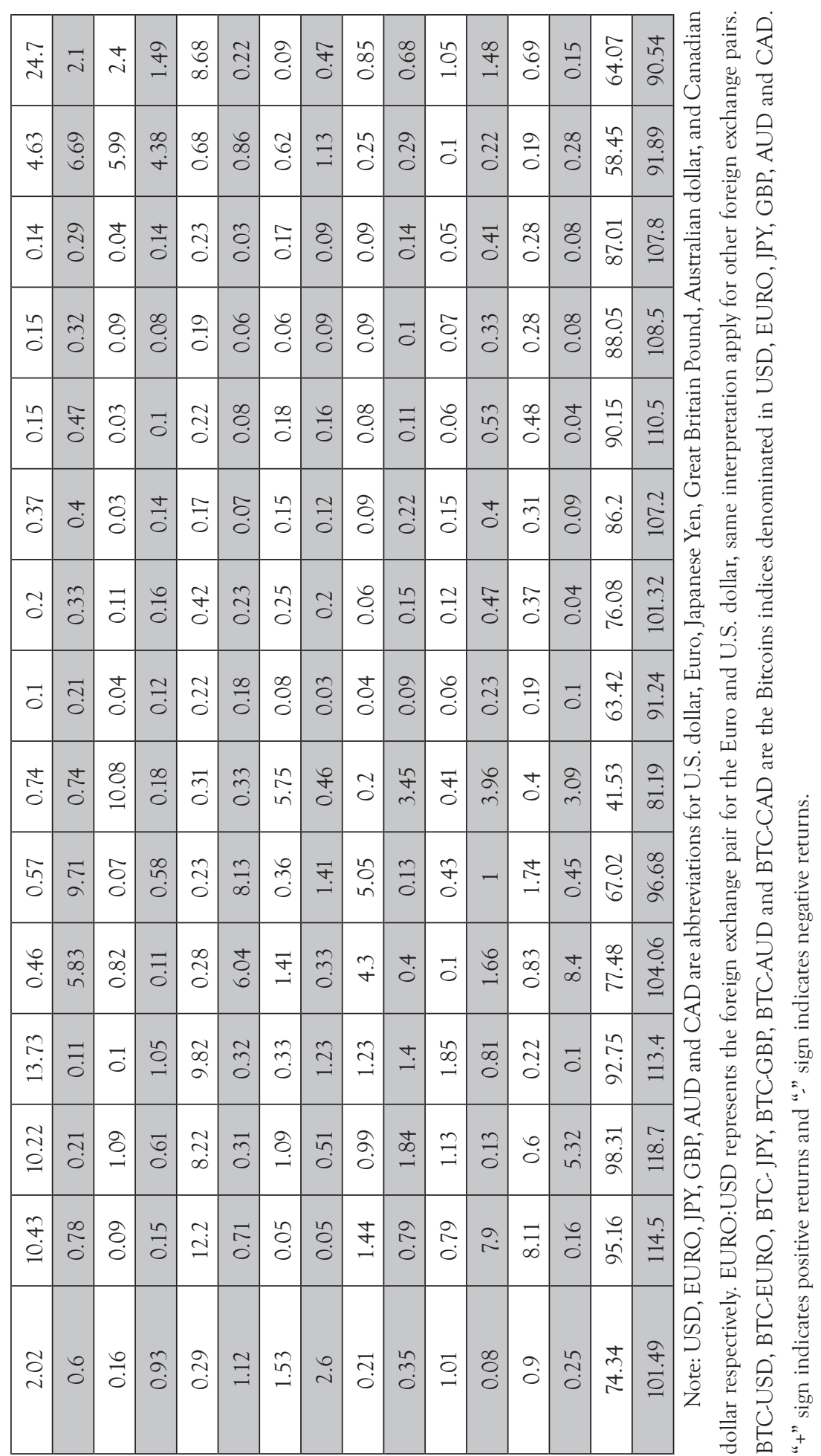




\begin{tabular}{|c|c|c|c|c|c|c|c|c|c|c|c|c|c|c|c|c|c|}
\hline 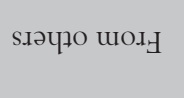 & $\begin{array}{l}\infty \\
\stackrel{0}{0} \\
\stackrel{i}{\sim}\end{array}$ & $\begin{array}{l}\stackrel{+}{ \pm} \\
\stackrel{ \pm}{ }\end{array}$ & $\begin{array}{l}\infty \\
\infty \\
\infty \\
\infty\end{array}$ & 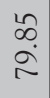 & $\begin{array}{l}\stackrel{\sim}{\sim} \\
\curvearrowright\end{array}$ & $\begin{array}{l}\tilde{n} \\
\stackrel{2}{\curvearrowright}\end{array}$ & 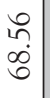 & $\begin{array}{l}n \\
0 \\
0 \\
i\end{array}$ & $\begin{array}{l}\hat{\sigma} \\
\hat{o}\end{array}$ & $\begin{array}{l}\tilde{J} \\
\dot{ \pm}\end{array}$ & $\begin{array}{l}0 \\
\stackrel{0}{0} \\
\stackrel{0}{0}\end{array}$ & $\begin{array}{l}\tilde{\infty} \\
\infty \\
\infty \\
0\end{array}$ & $\begin{array}{l}\infty \\
\sim \\
\\
n\end{array}$ & $\begin{array}{l}\stackrel{શ}{ \pm} \\
\underset{ \pm}{ \pm}\end{array}$ & $\begin{array}{l} \pm \\
\infty \\
\mathbb{N}\end{array}$ & $\begin{array}{l}\vec{b} \\
\dot{0} \\
\infty\end{array}$ & $\stackrel{8}{\stackrel{0}{2}}$ \\
\hline 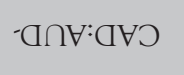 & $\stackrel{2}{2}$ & $\tilde{O}$ & $\begin{array}{l}2 \\
\stackrel{0}{0}\end{array}$ & $\begin{array}{l}\dot{0} \\
\stackrel{0}{0}\end{array}$ & $\begin{array}{l}\tilde{0} \\
0\end{array}$ & $\begin{array}{l}8 \\
0 \\
0\end{array}$ & $\begin{array}{l}\circ \\
\stackrel{0}{0}\end{array}$ & $\stackrel{\sim}{\longrightarrow}$ & $\stackrel{\mathfrak{f}}{\dot{0}}$ & $\begin{array}{l}\infty \\
0 \\
\infty \\
\infty\end{array}$ & $\stackrel{\overbrace{}}{\tilde{0}}$ & $\begin{array}{l}\vec{\nabla} \\
0\end{array}$ & $\stackrel{m}{0}$ & $\begin{array}{l}2 \\
\text { in } \\
\text { in }\end{array}$ & 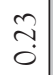 & $\stackrel{20}{\circ}$ & $\begin{array}{l}n \alpha \\
\grave{i}\end{array}$ \\
\hline 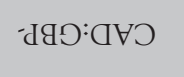 & $\begin{array}{l}\tilde{O} \\
\hat{O}\end{array}$ & $\overrightarrow{0}$ & $\overrightarrow{0}$ & 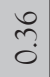 & $\begin{array}{l}\infty \\
\stackrel{0}{0} \\
0\end{array}$ & $\begin{array}{l}\tilde{0} \\
0\end{array}$ & $\begin{array}{l}\tilde{N} \\
\sim \\
\sim\end{array}$ & $\begin{array}{l}\vec{\sigma} \\
0\end{array}$ & $\begin{array}{l}\circ \\
\Xi \\
\Xi\end{array}$ & $\tilde{\sigma}$ & $\begin{array}{l}0 \\
\stackrel{0}{0}\end{array}$ & \begin{tabular}{l}
$\infty$ \\
\hdashline \\
0
\end{tabular} & 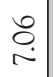 & 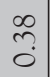 & $\stackrel{\sim}{\leftrightarrows}$ & సे & $\begin{array}{l}n \\
n \\
0\end{array}$ \\
\hline -dgD:વへサ & $\begin{array}{l}\widetilde{O} \\
\stackrel{0}{0}\end{array}$ & $\stackrel{2}{2}$ & $\begin{array}{l}+ \\
\stackrel{0}{0}\end{array}$ & $\tilde{n}$ & $\begin{array}{l}m \\
0 \\
0\end{array}$ & $\begin{array}{l} \pm \\
0 \\
0\end{array}$ & 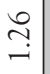 & $\tilde{0}$ & $\begin{array}{l}\vec{b} \\
\infty\end{array}$ & $\begin{array}{c}\tilde{0} \\
\tilde{0}\end{array}$ & $\stackrel{2}{\overrightarrow{0}}$ & $\begin{array}{l}\tilde{0} \\
0\end{array}$ & $\infty$ & $\begin{array}{l}\stackrel{2}{0} \\
0\end{array}$ & $\stackrel{\infty}{\rightarrow}$ & 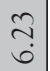 & $=$ \\
\hline 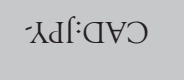 & oे. & $\begin{array}{l}\tilde{0} \\
0\end{array}$ & $\begin{array}{l}n \\
0 \\
0\end{array}$ & $\begin{array}{r}\tilde{0} \\
0\end{array}$ & $\begin{array}{l}\dot{0} \\
\dot{0}\end{array}$ & $\tilde{O}$ & 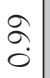 & $\begin{array}{l}\tilde{\partial} \\
\tilde{U}\end{array}$ & $\begin{array}{l}n \\
n \\
0\end{array}$ & $\stackrel{\sim}{0}$ & $\vec{\vartheta}$ & $\begin{array}{l}3 \\
\infty \\
\infty\end{array}$ & $\begin{array}{l}n \\
\tilde{0} \\
0\end{array}$ & $\begin{array}{l}\infty \\
\dddot{m} \\
0\end{array}$ & $\stackrel{\vartheta}{=}$ & $\overline{\check{o}}$ & $\tilde{\sigma}$ \\
\hline 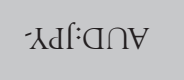 & $\stackrel{2}{3}$ & $\overrightarrow{0}$ & $\begin{array}{l} \pm \\
\\
0\end{array}$ & \begin{tabular}{l}
\multirow{0}{0}{} \\
0
\end{tabular} & $\stackrel{7}{0}$ & $\tilde{0}$ & $\tilde{0}$ & $\stackrel{\simeq}{a}$ & $\begin{array}{l}\circ \\
\stackrel{0}{0}\end{array}$ & $\stackrel{+}{ \pm}$ & $\begin{array}{l}\stackrel{2}{0} \\
\dot{0}\end{array}$ & $\stackrel{\tilde{a}}{a}$ & ڤે & $\tilde{\sigma}$ & $\tilde{\tilde{0}}$ & 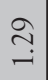 & \\
\hline -XdlःdGり & $\begin{array}{l}\stackrel{2}{\circ} \\
\stackrel{\circ}{0}\end{array}$ & $\begin{array}{l}\text { L̂ } \\
0\end{array}$ & $\overrightarrow{0}$ & $\begin{array}{l}8 \\
0 \\
0\end{array}$ & $\begin{array}{l}\tilde{O} \\
0\end{array}$ & $\begin{array}{l}\tilde{O} \\
0\end{array}$ & $\begin{array}{l}n \\
\tilde{0}\end{array}$ & $\begin{array}{l}\vec{\infty} \\
\dot{\Xi}\end{array}$ & $\vec{m}$ & $\tilde{\tilde{o}}$ & $\begin{array}{l}\tilde{f} \\
\dot{0}\end{array}$ & $\begin{array}{l}\infty \\
\infty \\
= \\
=\end{array}$ & $\stackrel{+}{-}$ & $\stackrel{\sim}{0}$ & $\underset{\Xi}{ \pm}$ & $\stackrel{f}{\leftarrow}$ & $\hat{o}$ \\
\hline 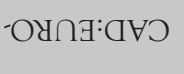 & ठே. & $\begin{array}{l}20 \\
0 \\
0\end{array}$ & $\tilde{O}$ & $\begin{array}{l}\tilde{O} \\
0 \\
0\end{array}$ & $\begin{array}{l}\tilde{O} \\
0 \\
0\end{array}$ & $\begin{array}{l} \pm \\
\stackrel{0}{0}\end{array}$ & 으 & $m$ & $\begin{array}{l}\tilde{D} \\
0 \\
0\end{array}$ & $\overrightarrow{0}$ & $\begin{array}{l}\infty \\
\stackrel{0}{0}\end{array}$ & $\begin{array}{c}\tilde{0} \\
0\end{array}$ & $\tilde{0}$ & $\stackrel{2}{=}$ & $\underset{\vec{\sim}}{\stackrel{\vec{N}}{1}}$ & $\stackrel{m}{\circ}$ & $\stackrel{\overbrace{}}{\grave{0}}$ \\
\hline 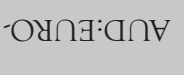 & $\begin{array}{l}\widetilde{O} \\
\stackrel{0}{0}\end{array}$ & $\tilde{O}$ & ¿̊. & $\begin{array}{l}\tilde{O} \\
0\end{array}$ & $\begin{array}{l}8 \\
0 \\
0\end{array}$ & $\begin{array}{l}+ \\
O \\
0\end{array}$ & $\begin{array}{l}\tilde{b} \\
0 \\
0\end{array}$ & $\stackrel{\sim}{\stackrel{\sim}{-}}$ & $\vec{n}$ & $\stackrel{\overbrace{}}{\rightarrow}$ & $\tilde{\tilde{O}}$ & $\begin{array}{l}\vec{\sim} \\
\tilde{0}\end{array}$ & ફे. & $\begin{array}{l}+ \\
\infty \\
-\end{array}$ & $\stackrel{9}{\rightarrow}$ & $\frac{\square}{0}$ & $\begin{array}{l}\Omega \\
\infty \\
0\end{array}$ \\
\hline 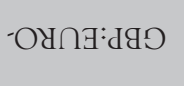 & مْ & $\begin{array}{l}0 \\
0 \\
0\end{array}$ & $\begin{array}{l}\infty \\
\stackrel{0}{0} \\
0\end{array}$ & $\begin{array}{l}n \\
0 \\
0\end{array}$ & $\begin{array}{l}\sqcup \\
0 \\
0\end{array}$ & $\begin{array}{l}n \\
\stackrel{n}{0} \\
0\end{array}$ & $\begin{array}{l}\dot{0} \\
\dot{0} \\
0\end{array}$ & $\underset{\sim}{\stackrel{\sim}{\sim}}$ & $\begin{array}{l}+ \\
\dot{*}\end{array}$ & 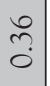 & $\begin{array}{l}0 \\
\stackrel{0}{0}\end{array}$ & 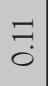 & 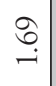 & 華 & $\begin{array}{l}\stackrel{0}{0} \\
\stackrel{0}{0}\end{array}$ & $\begin{array}{l}+ \\
\dot{5}\end{array}$ & $\stackrel{2}{\circ}$ \\
\hline - O४⿻コः:גd & $\stackrel{7}{7}$ & 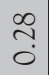 & $\begin{array}{l}\text { ठे. } \\
\dot{0}\end{array}$ & in & $\tilde{\tilde{0}}$ & 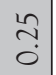 & 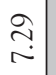 & $\begin{array}{l}n \\
\infty \\
0 \\
0\end{array}$ & $\begin{array}{c}\overrightarrow{0} \\
\text { in }\end{array}$ & $\stackrel{\sim}{\stackrel{\sim}{\rightarrow}}$ & $\begin{array}{c}\overrightarrow{0} \\
i\end{array}$ & $\begin{array}{l}\infty \\
\vec{\sim}\end{array}$ & $\tilde{m}$ & $\begin{array}{l}n \\
\stackrel{2}{0} \\
0\end{array}$ & $\exists$ & $\begin{array}{l}\stackrel{2}{a} \\
a\end{array}$ & $\begin{array}{l}n \\
0 \\
0\end{array}$ \\
\hline 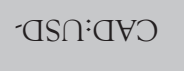 & $\begin{array}{l}\widetilde{O} \\
\stackrel{0}{0}\end{array}$ & $\stackrel{2}{2} 0$ & $\tilde{O}$ & $\hat{O}$ & $\begin{array}{l}20 \\
0 \\
0\end{array}$ & $\begin{array}{l}\infty \\
0 \\
0\end{array}$ & $\begin{array}{l}0 \\
+ \\
0\end{array}$ & $\tilde{0}$ & 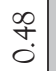 & $\stackrel{\overbrace{}}{\rightarrow}$ & $\underset{\sim}{\stackrel{N}{\sim}}$ & $\stackrel{?}{-}$ & $\underset{\sim}{\stackrel{ \pm}{0}}$ & $\tilde{0}$ & $\begin{array}{l}0 \\
\infty \\
0\end{array}$ & $\stackrel{\infty}{\rightarrow}$ & $\vec{F}$ \\
\hline 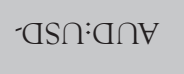 & $\begin{array}{l}n \\
0 \\
0\end{array}$ & $\tilde{0}$ & $\hat{O}$ & $\begin{array}{l}\tilde{O} \\
0 \\
0\end{array}$ & $\tilde{0}$ & $\begin{array}{l}\tilde{O} \\
\tilde{0}\end{array}$ & $\stackrel{2}{2}$ & $\dot{0}$ & $\begin{array}{l}\approx \\
\tilde{0}\end{array}$ & $\vec{\sim}$ & $\stackrel{t}{\stackrel{t}{-}}$ & $\stackrel{\text { จे }}{1}$ & $\begin{array}{l}\infty \\
\\
0\end{array}$ & $\stackrel{\text { ڤ̊ }}{\circ}$ & $\begin{array}{l}\infty \\
0 \\
0\end{array}$ & $\stackrel{\text { సे }}{0}$ & $\begin{array}{l}n \\
\infty \\
0 \\
0\end{array}$ \\
\hline -đSก:dgり & $\begin{array}{l}n \\
0 \\
0\end{array}$ & $\stackrel{\text { Lo }}{0}$ & $\tilde{0}$ & $\begin{array}{l}8 \\
0 \\
0\end{array}$ & $\begin{array}{l} \pm \\
\\
0\end{array}$ & $\begin{array}{l} \pm \\
0 \\
0\end{array}$ & $\tilde{n}$ & 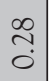 & $\underset{亡}{\stackrel{ \pm}{ \pm}}$ & $\begin{array}{l}\tilde{\sigma} \\
\dot{0}\end{array}$ & $\begin{array}{c}\tilde{0} \\
\infty \\
0\end{array}$ & $\begin{array}{c}\tilde{\sim} \\
i\end{array}$ & $\begin{array}{l}0 \\
+ \\
0\end{array}$ & $\stackrel{\sim}{0}$ & $\tilde{0}$ & $\begin{array}{l}n \\
n \\
0\end{array}$ & $\stackrel{m}{0}$ \\
\hline
\end{tabular}




\begin{tabular}{|c|c|c|c|c|c|c|c|c|c|c|c|c|c|c|c|c|c|c|c|c|c|}
\hline$\stackrel{n}{2}$ & $\begin{array}{l}\vec{\nabla} \\
\stackrel{\sim}{*}\end{array}$ & ڤે & $\begin{array}{l}\tilde{n} \\
\tilde{8} \\
\tilde{8}\end{array}$ & $\stackrel{\infty}{\underset{N}{i}}$ & $\begin{array}{l}0 \\
\stackrel{+}{ \pm} \\
\end{array}$ & $\begin{array}{l}\stackrel{+}{0} \\
\stackrel{2}{i}\end{array}$ & $\begin{array}{l}\stackrel{\vartheta}{0} \\
\stackrel{2}{\alpha}\end{array}$ & $\begin{array}{l}i \\
2 \\
2\end{array}$ & 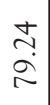 & $\begin{array}{l}0 \\
\stackrel{2}{0} \\
8 \\
8\end{array}$ & $\begin{array}{l}\dot{v} \\
\grave{n}\end{array}$ & $\begin{array}{l}\stackrel{0}{+} \\
\text { oे }\end{array}$ & 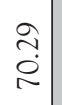 & $\stackrel{+}{\dot{\theta}}$ & $\begin{array}{l}\vec{n} \\
i n \\
n\end{array}$ & $\underset{⿱ 亠 凶}{+}$ & $\stackrel{\infty}{\stackrel{m}{\Gamma}}$ & $\underset{I}{\stackrel{\vec{I}}{\sim}}$ & $\underset{n}{\stackrel{\infty}{n}}$ & $\begin{array}{l}\vec{\infty} \\
i \\
i\end{array}$ & $\begin{array}{l}\hat{n} \\
i n \\
n\end{array}$ \\
\hline$\stackrel{\overrightarrow{0}}{0}$ & $\underset{6}{7}$ & fั & $\stackrel{\sim}{\stackrel{\sim}{\sim}}$ & $\tilde{O}$ & $\stackrel{2}{2}$ & $\begin{array}{l}\widetilde{O} \\
\stackrel{0}{0}\end{array}$ & $\begin{array}{l}\tilde{O} \\
0 \\
0\end{array}$ & $\begin{array}{l}\text { ¿ } \\
0\end{array}$ & $\begin{array}{l}\tilde{O} \\
0 \\
0\end{array}$ & 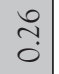 & $\hat{\tilde{o}}$ & $\tilde{0}$ & 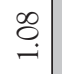 & $\begin{array}{c}\vec{\sigma} \\
\dot{m}\end{array}$ & $\stackrel{\grave{0}}{\grave{0}}$ & $\tilde{0}$ & $\begin{array}{l}0 \\
\stackrel{2}{0}\end{array}$ & $\begin{array}{l}\widetilde{\sigma} \\
\dot{+}\end{array}$ & $\stackrel{+}{\tilde{O}}$ & $\stackrel{9}{0}$ & $\hat{n}$ \\
\hline$\stackrel{⿱}{\Im}$ & $\hat{o}$ & کٌ & $\begin{array}{l}\tilde{b} \\
0\end{array}$ & ๐̊ & "̈ & $\overrightarrow{0}$ & $\stackrel{\sim}{0}$ & "̈ & $\begin{array}{l}\infty \\
0 \\
0 \\
0\end{array}$ & $\stackrel{\sim}{3}$ & $\begin{array}{l}0 \\
0 \\
0 \\
0\end{array}$ & $\hat{\circ}$ & $\stackrel{\overrightarrow{0}}{0}$ & $\underset{⿱ ㇒}{ \pm}$ & $\tilde{\sim}$ & 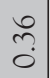 & $\begin{array}{l}\widetilde{C} \\
\text { U }\end{array}$ & $r$ & $\tilde{\tilde{0}}$ & 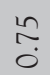 & $\frac{n}{m}$ \\
\hline$\stackrel{2}{\simeq}$ & $\stackrel{શ}{\stackrel{શ}{i}}$ & $\underset{\text { I }}{\text { I }}$ & $\frac{9}{6}$ & $\exists$ & $\stackrel{0}{\circ}$ & $\ddot{0}$ & $\overrightarrow{\widetilde{O}}$ & ठे. & $\begin{array}{l}0 \\
0 \\
0\end{array}$ & $\stackrel{n}{?}$ & $\stackrel{\vartheta}{\mathfrak{\theta}}$ & $\tilde{0}$ & $\stackrel{n}{\stackrel{n}{0}}$ & $\stackrel{\eta}{-}$ & $\begin{array}{l}\tilde{n} \\
\sim\end{array}$ & $\tilde{\tilde{0}}$ & $\sim$ & $\stackrel{n}{\sim}$ & $\tilde{n}$ & $\stackrel{n}{\stackrel{n}{i}}$ & $\stackrel{n}{\hat{0}}$ \\
\hline & oे & mे. & $\underset{\Im}{\tilde{O}}$ & $\tilde{o}_{0}$ & $\begin{array}{l}8 \\
0 \\
0\end{array}$ & $\stackrel{7}{0}$ & $\stackrel{+}{0}$ & $\begin{array}{l}\text { ¿ } \\
0\end{array}$ & $\begin{array}{l}+ \\
\dot{0} \\
\dot{0}\end{array}$ & $\stackrel{\infty}{\dddot{0}}$ & $\rightleftarrows$ & Ő & $\stackrel{n}{\stackrel{n}{*}}$ & 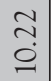 & $\begin{array}{l}\infty \\
\infty \\
0\end{array}$ & 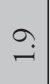 & $\overrightarrow{\widehat{\sim}}$ & 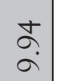 & $\stackrel{\vec{a}}{a}$ & $\begin{array}{l}\tilde{\sigma} \\
=\end{array}$ & $\begin{array}{l}\stackrel{0}{2} \\
\text { స }\end{array}$ \\
\hline 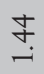 & 广ัণ & $\stackrel{m}{0}$ & $\begin{array}{l}\infty \\
\stackrel{\infty}{0} \\
\text { in }\end{array}$ & $\stackrel{\text { Oै }}{\circ}$ & $\hat{O}$ & $\stackrel{7}{\dddot{0}}$ & $\begin{array}{l}+ \\
\end{array}$ & $\begin{array}{l}\text { Oै } \\
0\end{array}$ & ऽ̊ & $\stackrel{n}{\circ}$ & $\begin{array}{l}\infty \\
\infty \\
0 \\
0\end{array}$ & $\stackrel{t}{\sigma}$ & $\begin{array}{l}\stackrel{\alpha}{\infty} \\
\infty\end{array}$ & $\stackrel{\widetilde{\sim}}{\stackrel{+}{*}}$ & $\underset{ت}{ \pm}$ & 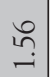 & $\tilde{n}$ & $\stackrel{\sim}{m}$ & $\stackrel{\sim}{\stackrel{n}{n}}$ & $\stackrel{n}{\stackrel{n}{i}}$ & 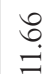 \\
\hline$\stackrel{\Xi}{\Xi}$ & $\stackrel{\tilde{n}}{\stackrel{+}{*}}$ & $\hat{\text { nे }}$ & $\stackrel{\overbrace{}}{0}$ & $\stackrel{\circ}{\circ}$ & $\begin{array}{l}\tilde{O} \\
0 \\
0\end{array}$ & $\stackrel{n}{0}$ & Oั & $\begin{array}{l}+ \\
O \\
0\end{array}$ & $\begin{array}{l}20 \\
0 \\
0\end{array}$ & 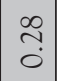 & $\begin{array}{l}2 \\
\infty \\
0\end{array}$ & \begin{tabular}{l} 
\pm \\
\multirow{\sigma}{*}{}
\end{tabular} & $\underset{0}{\stackrel{N}{0}}$ & $\stackrel{\infty}{\stackrel{\infty}{\rightarrow}}$ & $\underset{\Xi}{\Xi}$ & 六. & $\begin{array}{l}\tilde{0} \\
0 \\
0\end{array}$ & $\bar{\sigma}$ & $\begin{array}{l}\infty \\
\stackrel{\infty}{\sim} \\
\stackrel{\sim}{\sim}\end{array}$ & $\stackrel{n}{\curvearrowright}$ & $\begin{array}{l}+ \\
\infty \\
\infty \\
\infty\end{array}$ \\
\hline$\tilde{0}$ & $\stackrel{0}{\circ}$ & $\exists$ & $\begin{array}{l}\infty \\
0 \\
0\end{array}$ & ठ઼. & $\exists$ & $\begin{array}{l}\infty \\
\stackrel{0}{0} \\
0\end{array}$ & $\stackrel{ \pm}{\ddagger}$ & $\exists$ & $\exists$ & $\stackrel{\simeq}{\rightleftarrows}$ & 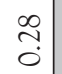 & $\begin{array}{l}2 \\
\stackrel{2}{0} \\
0\end{array}$ & $\begin{array}{l}\vec{t} \\
0\end{array}$ & $\underset{\pi}{\overparen{T}}$ & $\underset{ \pm}{\stackrel{ \pm}{ \pm}}$ & $\stackrel{m}{\dot{y}}$ & $\begin{array}{l}\overrightarrow{0} \\
\dot{0}\end{array}$ & 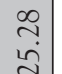 & $\begin{array}{l}+ \\
\infty \\
0\end{array}$ & ฉे & $\begin{array}{l}\infty \\
\infty \\
\infty \\
\infty\end{array}$ \\
\hline$\stackrel{\infty}{\circ}$ & $\stackrel{\circ}{\stackrel{\sim}{ }}$ & ठे. & $\stackrel{\infty}{\sim}$ & $\stackrel{m}{0}$ & $\stackrel{\sim}{\dddot{0}}$ & oे & $\stackrel{ \pm}{\leftrightarrows}$ & ठे. & $\stackrel{2}{\circ}$ & $\stackrel{\infty}{\stackrel{\infty}{0}}$ & $\ddot{0}$ & 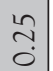 & $\begin{array}{l}\infty \\
\stackrel{n}{\sim}\end{array}$ & $\stackrel{\leftrightarrow}{-}$ & 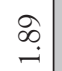 & ஜे & $\begin{array}{l}\vec{F} \\
\dot{0}\end{array}$ & $\stackrel{\text { In }}{\circ}$ & กี & すั & ते \\
\hline$\stackrel{\Xi}{\leftrightarrows}$ & $\begin{array}{l}\vec{\infty} \\
\dot{+}\end{array}$ & Oِ & $\stackrel{n}{f}$ & రิ & $\tilde{O}$ & $\stackrel{7}{0}$ & $\stackrel{5}{0}$ & $\underset{0}{ \pm}$ & \begin{tabular}{l}
0 \\
\hdashline \\
0
\end{tabular} & $\begin{array}{l}0 \\
+ \\
0\end{array}$ & $\stackrel{\partial}{0}$ & ふे & $\stackrel{m}{\stackrel{n}{0}}$ & ஸे. & $\stackrel{0}{+}$ & స్ & $\stackrel{?}{+}$ & $\begin{array}{l}\underset{+}{\sigma} \\
\stackrel{+}{*}\end{array}$ & $\hat{\sigma}$ & $\vec{\vartheta}$ & $\stackrel{?}{\rightarrow}$ \\
\hline$\stackrel{n}{\stackrel{2}{\infty}} \underset{\infty}{\infty}$ & $\stackrel{\infty}{\infty}$ & ठे. & ָ̃ & Ő & $\tilde{O}$ & $\stackrel{\sigma}{0}$ & oे. & ธ̊. & $\begin{array}{l}8 \\
0 \\
0\end{array}$ & $\stackrel{\text { Iै }}{0}$ & テ⿱宀⿻⺀大 & $\stackrel{2}{0}$ & oे & $\overrightarrow{\widetilde{0}}$ & $\begin{array}{l}\stackrel{\tilde{n}}{\mathrm{~N}} \\
\stackrel{\sim}{ }\end{array}$ & 市 & $\underset{\sim}{ \pm}$ & $\stackrel{\varkappa}{-}$ & $\stackrel{\Xi}{\rightleftarrows}$ & $\begin{array}{l}0 \\
\infty \\
0 \\
0\end{array}$ & $\begin{array}{l}+ \\
\text { Oे } \\
0\end{array}$ \\
\hline$\stackrel{\mathscr{n}}{\overparen{0}}$ & $\stackrel{\sim}{\circ}$ & $\begin{array}{l}\infty \\
\stackrel{n}{0} \\
0\end{array}$ & $\underset{0}{F}$ & $\stackrel{\infty}{\circ}$ & ठे. & $\ddot{0}$ & $\stackrel{7}{\circ}$ & $\dddot{0}$ & $\stackrel{7}{\dddot{0}}$ & $\begin{array}{l}6 \\
\dot{+}\end{array}$ & กี & $\begin{array}{l}\stackrel{T}{ \pm} \\
\text { in }\end{array}$ & $\hat{o}$ & $\begin{array}{l}n \\
0 \\
0\end{array}$ & $\stackrel{\vartheta}{0}$ & $\stackrel{\Re}{\check{0}}$ & $\begin{array}{l}\circ \\
\stackrel{\circ}{\infty} \\
-\end{array}$ & ก̂? & 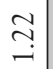 & $\underset{\sim}{\tilde{m}}$ & $\stackrel{\stackrel{+}{+}}{\sim}$ \\
\hline$\stackrel{\text { İ }}{0}$ & $\begin{array}{l}\vec{\infty} \\
0 \\
0\end{array}$ & 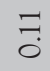 & 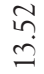 & $\stackrel{ \pm}{ \pm}$ & $\vec{\jmath}$ & $\overrightarrow{0}$ & $\stackrel{?}{0}$ & $\stackrel{?}{0}$ & $\exists$ & Oָ & $\underset{\sim}{\sim}$ & $\begin{array}{l}\infty \\
\infty \\
\text { in }\end{array}$ & $\underset{\tilde{0}}{\tilde{1}}$ & $\begin{array}{l}\stackrel{a}{\pi} \\
a\end{array}$ & $\stackrel{+}{n}$ & $\stackrel{0}{+}$ & $\underset{\sim}{\stackrel{+}{+}}$ & $\begin{array}{l}\text { in } \\
0 \\
0\end{array}$ & $\begin{array}{l}\infty \\
\stackrel{n}{n} \\
0\end{array}$ & $\vec{\sim}$ & $\stackrel{\infty}{\circ}$ \\
\hline$\frac{m}{0}$ & $\begin{array}{l}\text { t' } \\
\text { in }\end{array}$ & $\stackrel{\text { ै. }}{\circ}$ & - & $\stackrel{\pi}{0}$ & 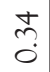 & $\stackrel{\text { \ે }}{0}$ & $\tilde{\tilde{0}}$ & $\stackrel{\infty}{\sim}$ & $\stackrel{2}{\mathfrak{2}}$ & $\underset{\sim}{\sim}$ & $\stackrel{\sim}{\infty}$ & 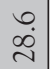 & $\begin{array}{l}\stackrel{2}{\sim} \\
i n\end{array}$ & $\begin{array}{l}0 \\
\dot{1}\end{array}$ & $\overrightarrow{\tilde{o}}$ & $\begin{array}{l}i n \\
i n \\
\infty\end{array}$ & $\tilde{n}$ & $\tilde{n}$ & $\stackrel{\infty}{\sim}$ & $\stackrel{\infty}{\stackrel{\sim}{\longrightarrow}}$ & $\begin{array}{l}\stackrel{2}{0} \\
0\end{array}$ \\
\hline
\end{tabular}




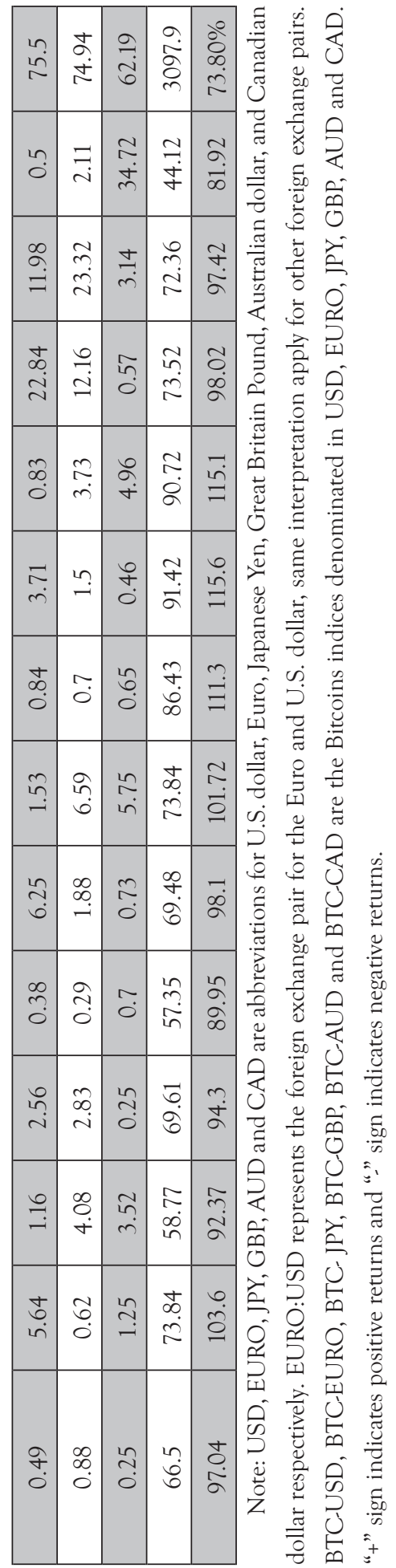




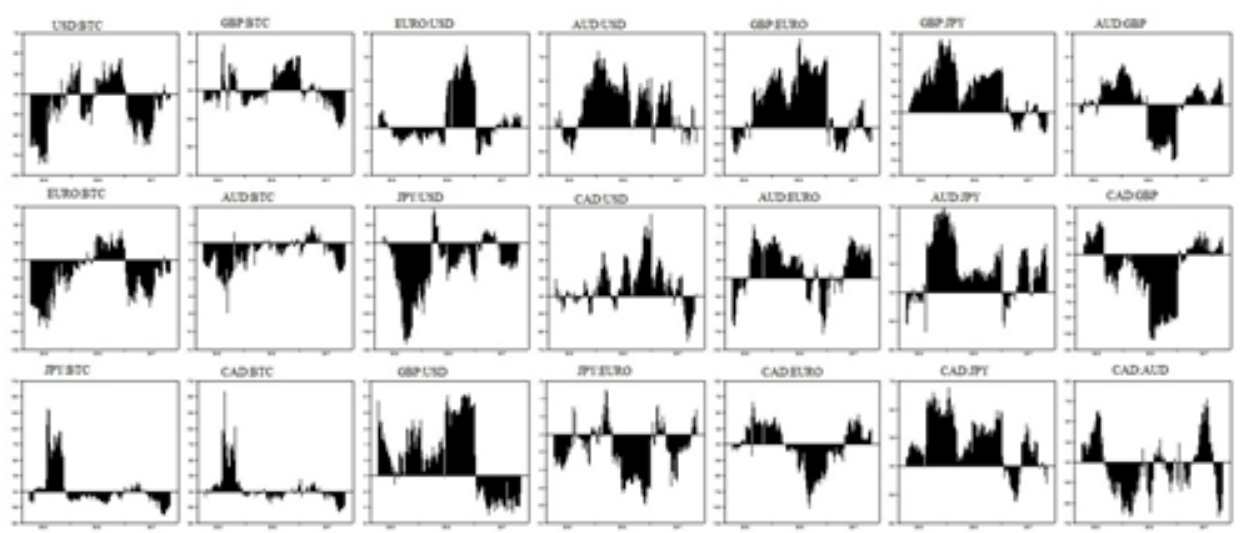

Figure 2: Returns' "FROM others” Directional Spillover Asymmetry Measures $S A M_{2 N, i \rightarrow}^{H}$

ered as transmitter of net positive spillover to others. For the markets for which "TO others SAM" is negative indicates transmission of excess negative spillover and are considered as transmitter of net negative spillover. The findings of "TO others SAM" return spillover (figure 3) reveals that AUD:USD, GBP:EURO, AUD:EURO, CAD:EURO, GBP:JPY, AUD:JPY, and CAD:JPY, AUD:GBP, and CAD:GBP transmitted net positive return spillover and BTC:USD, EURO:BTC, JPY:BTC, GBP:BTC, AUD:BTC, CAD:BTC, EURO:USD, JPY:USD, GBP:USD, CAD:USD, JPY:EURO, and CAD:AUD transmitted net negative return spillover to other during the sampled period. The highest net positive spillover to other is transmitted by AUD:EURO (13.15) and the lowest net positive spillover to others is transmitted by CAD:GBP (1.26). The highest net negative spillover to others is transmitted by JPY:USD (-10.22) and the lowest net negative spillover is transmitted by GBP:BTC (-2.34).

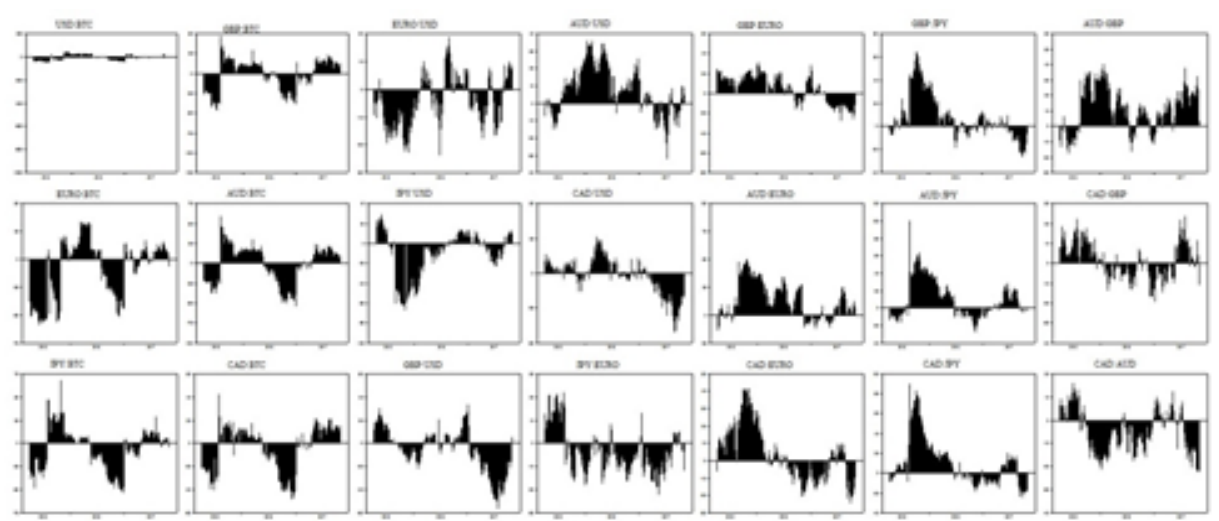

Figure 3: Returns' “TO others” Directional Spillover Asymmetry Measures, $S A M_{2 N, i \leftarrow}^{H}$ 


\begin{tabular}{|c|c|c|c|c|c|c|c|c|c|c|c|c|c|}
\hline 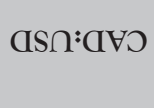 & $\begin{array}{l}\text { to } \\
\text { Oे }\end{array}$ & $\stackrel{\infty}{0}$ & ธิ & $\stackrel{8}{\circ}$ & $\stackrel{\circ}{\circ}$ & $\stackrel{m}{0}$ & 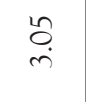 & $\tilde{\tilde{0}}$ & $\stackrel{+}{m}$ & $\begin{array}{l}\overbrace{}^{\circ} \\
\infty\end{array}$ & $\begin{array}{l}\tilde{n} \\
\tilde{n}\end{array}$ & $\stackrel{\infty}{\circ}$ & $\stackrel{ \pm}{\circlearrowleft}$ \\
\hline đSA: $\mathrm{A} \cap \forall$ & $\ddot{0}$ & $\begin{array}{l}\text { to } \\
0 \\
0\end{array}$ & $\stackrel{\text { Oे. }}{0}$ & $\tilde{o}$ & $\stackrel{n}{0}$ & $\tilde{O}$ & $\begin{array}{l}\stackrel{\sim}{+} \\
\stackrel{+}{*}\end{array}$ & $\cong$ & $\stackrel{\infty}{\underset{+}{+}}$ & $\stackrel{n}{\sim}$ & 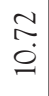 & ฑิ & $\tilde{0}$ \\
\hline 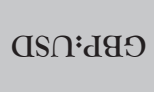 & $\hat{O}$ & $\stackrel{0}{\because}$ & ஓे. & $\stackrel{\infty}{+}$ & $\stackrel{7}{0}$ & 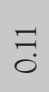 & $\underset{\sim}{\sim}$ & $\stackrel{n}{?}$ & $\stackrel{\text { f }}{\dot{\sim}}$ & ஓे & $\stackrel{\sim}{*}$ & $\begin{array}{l}\tilde{n} \\
\text { nे }\end{array}$ & $\underset{\sim}{\widetilde{\sigma}}$ \\
\hline GS $\cap: X \mathrm{~d} l$ & ठ઼. & $\tilde{O}$ & $\stackrel{7}{7}$ & $\stackrel{+}{\circ}$ & $\stackrel{2}{\circ}$ & $\hat{o}$ & $\underset{+}{\stackrel{ }{\sim}}$ & $\begin{array}{l}\infty \\
\text { i } \\
\text { N }\end{array}$ & $\frac{9}{0}$ & $\stackrel{\text { }}{\circ}$ & 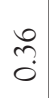 & $\underset{\sim}{\stackrel{\sim}{\sim}}$ & $\begin{array}{l}\tilde{\sigma} \\
i\end{array}$ \\
\hline 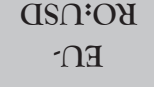 & 0 & $\stackrel{\widetilde{\jmath}}{\tilde{0}}$ & $\stackrel{\infty}{\circ}$ & $\stackrel{7}{\circ}$ & $\stackrel{2}{0}$ & $\stackrel{\text { L }}{0}$ & $\ddot{n}$ & $\begin{array}{l}+ \\
\infty \\
\dot{m}\end{array}$ & $\begin{array}{l}\infty \\
0 \\
\dot{1} \\
\end{array}$ & $\begin{array}{l}n \\
\infty \\
m \\
\dot{m}\end{array}$ & $\begin{array}{l}\underset{v}{+} \\
\dot{m}\end{array}$ & $\begin{array}{l}\infty \\
\stackrel{\infty}{0} \\
i n\end{array}$ & $\tilde{n}$ \\
\hline OLG: GVO & $\begin{array}{l}\infty \\
\infty \\
\infty \\
\sigma\end{array}$ & ஸे. & $\begin{array}{l}\stackrel{7}{*} \\
\stackrel{n}{n}\end{array}$ & $\begin{array}{l}\hat{0} \\
\text { in }\end{array}$ & $\frac{n}{10}$ & 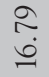 & $\stackrel{+}{0}$ & $\overrightarrow{0}$ & $\stackrel{\widetilde{̣}}{0}$ & $\hat{O}$ & $\overrightarrow{\widetilde{o}}$ & $\begin{array}{l}\tilde{0} \\
0\end{array}$ & 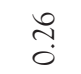 \\
\hline OLG: AกV & $\begin{array}{l}\infty \\
\infty \\
\circ\end{array}$ & กै. & $\begin{array}{l}\infty \\
\dot{ \pm}\end{array}$ & $\begin{array}{l}\stackrel{\circ}{\circ} \\
\dot{\Xi}\end{array}$ & 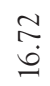 & $\stackrel{2}{\stackrel{2}{n}}$ & $\begin{array}{l}\infty \\
\stackrel{0}{0}\end{array}$ & $\begin{array}{l}\tilde{O} \\
0\end{array}$ & $\tilde{\tilde{o}}$ & $\stackrel{\sim}{\circ}$ & $\begin{array}{l}0 \\
0 \\
0\end{array}$ & $\begin{array}{l}\infty \\
\stackrel{0}{0} \\
0\end{array}$ & $\hat{\jmath}$ \\
\hline OLG:dGO & $\begin{array}{l}\infty \\
\infty \\
\infty\end{array}$ & テ⿱宀㠯 & $\stackrel{\Xi}{i}$ & $\stackrel{\substack{\sim \\
ٍ}}{ }$ & $\stackrel{n}{n}$ & 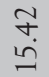 & $\stackrel{\infty}{\dddot{0}}$ & 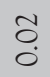 & $\stackrel{\curvearrowright}{\overparen{0}}$ & $\tilde{o}$ & $\overrightarrow{0}$ & $\underset{\sim}{\widetilde{0}}$ & in \\
\hline OLG:Xd & $\begin{array}{l}\hat{\circ} \\
\text { aे }\end{array}$ & 둥 & $\stackrel{\rightrightarrows}{\leftrightarrows}$ & $\begin{array}{l}\infty \\
\dot{\Xi}\end{array}$ & $\begin{array}{l}\vec{\infty} \\
\dot{ \pm}\end{array}$ & in & $\stackrel{\text { Ln. }}{0}$ & $\stackrel{3}{0}$ & 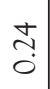 & 0 & $\begin{array}{l}\tilde{0} \\
0\end{array}$ & $\begin{array}{l}\tilde{O} \\
0\end{array}$ & 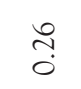 \\
\hline $\begin{array}{c}\text { OLGःOY } \\
\text {-คA }\end{array}$ & $\begin{array}{l}\bar{\sigma} \\
\stackrel{\Xi}{-}\end{array}$ & $\begin{array}{l}\dot{+} \\
\stackrel{2}{0} \\
\infty\end{array}$ & $\underset{\infty}{\stackrel{\sim}{*}}$ & $\underset{\sim}{\stackrel{\infty}{\infty}}$ & $\underset{\infty}{\simeq}$ & $\stackrel{\rightarrow}{\infty}$ & F. & $\tilde{O}$ & $\stackrel{n}{n}$ & $\overrightarrow{0}$ & $\stackrel{\sim}{\longrightarrow}$ & $\stackrel{\infty}{\circ}$ & $\stackrel{\overbrace{}}{\circ}$ \\
\hline GSAः OLG & 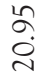 & $\stackrel{2}{\stackrel{2}{n}}$ & 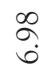 & 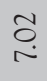 & $\stackrel{n}{2}$ & ْ & $\stackrel{\tilde{o}}{0}$ & $\overrightarrow{0}$ & $\stackrel{7}{0}$ & $\stackrel{\circ}{\circ}$ & $\tilde{O}$ & $\begin{array}{l}\tilde{O} \\
0\end{array}$ & $\stackrel{\sim}{\circ}$ \\
\hline 1. & $\begin{array}{l}\stackrel{\theta}{S} \\
\stackrel{0}{0} \\
\ddot{\bullet}\end{array}$ & 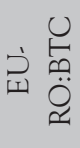 & 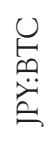 & $\begin{array}{l}\cup \\
\stackrel{0}{0} \\
\ddot{\ddot{n}} \\
0\end{array}$ & 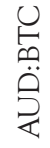 & 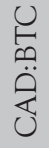 & D. & 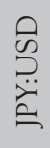 & $\begin{array}{l}\theta \\
\stackrel{0}{0} \\
\ddot{0} \\
0 \\
0\end{array}$ & 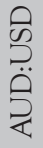 & 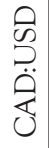 & 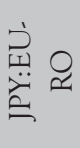 & 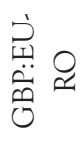 \\
\hline
\end{tabular}




\begin{tabular}{|c|c|c|c|c|c|c|c|c|c|c|c|c|c|c|c|c|c|}
\hline$\stackrel{⿱ 亠}{\underset{\sim}{-}}$ & $\begin{array}{l}\hat{n} \\
\text { in }\end{array}$ & $\begin{array}{c}\tilde{\infty} \\
0 \\
0\end{array}$ & $\begin{array}{l}\stackrel{0}{ } \\
\dot{m}\end{array}$ & 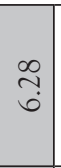 & $\stackrel{\circ}{\dot{o}}$ & $\begin{array}{l}\triangleright \\
\infty \\
\stackrel{\sim}{ }\end{array}$ & $\begin{array}{l}\widetilde{\sigma} \\
\mathrm{i}\end{array}$ & $\stackrel{\sim}{\rightarrow}$ & ڤેస & 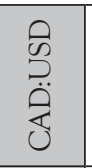 & $\begin{array}{l}\overrightarrow{0} \\
\dot{0}\end{array}$ & $\begin{array}{l}\tilde{o} \\
0\end{array}$ & $\begin{array}{l}\overrightarrow{0} \\
\dot{0}\end{array}$ & $\begin{array}{l}\tilde{\delta} \\
\delta\end{array}$ & $\begin{array}{l}2 \\
0 \\
0 \\
0\end{array}$ & $\begin{array}{l}\tilde{0} \\
0 \\
0\end{array}$ & $\stackrel{\infty}{\underset{\eta}{\rightarrow}}$ \\
\hline$\stackrel{\infty}{\stackrel{\infty}{\sim}}$ & $\begin{array}{l}\vec{\sigma} \\
\stackrel{\circ}{\circ}\end{array}$ & กี & $\stackrel{\stackrel{\sim}{\sim}}{\sim}$ & $\stackrel{\sim}{\stackrel{?}{=}}$ & $\begin{array}{l}\infty \\
\infty \\
\text { in }\end{array}$ & 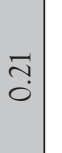 & 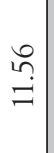 & $\begin{array}{l}\hat{b} \\
i\end{array}$ & $\hat{n}$ & 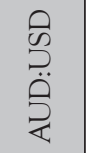 & $\begin{array}{l}\widetilde{\sigma} \\
\dot{O}\end{array}$ & $\begin{array}{l}8 \\
0\end{array}$ & $\begin{array}{l}\overrightarrow{0} \\
0\end{array}$ & $\begin{array}{l}\widetilde{O} \\
\stackrel{0}{\circ}\end{array}$ & $\begin{array}{l}\infty \\
0 \\
0 \\
0\end{array}$ & $\begin{array}{l}\tilde{0} \\
0 \\
0\end{array}$ & $\stackrel{+}{+}$ \\
\hline$\stackrel{\infty}{\circ}$ & $\begin{array}{l}n \\
n \\
0\end{array}$ & $\begin{array}{l}\underset{\infty}{\sim} \\
\infty\end{array}$ & $\underset{\sim}{\stackrel{\sim}{\rightarrow}}$ & \begin{tabular}{l}
\multirow{1}{0}{} \\
0
\end{tabular} & 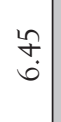 & 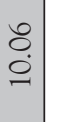 & $\tilde{n}$ & $\begin{array}{l}\infty \\
0 \\
\sim \\
ن\end{array}$ & $\begin{array}{l}\bar{n} \\
\tilde{n}\end{array}$ & $\begin{array}{l}\frac{w}{n} \\
\stackrel{\tilde{n}}{0} \\
0\end{array}$ & $\tilde{O}$ & $\overrightarrow{0}$ & $\begin{array}{l}\tilde{0} \\
0\end{array}$ & 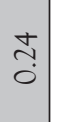 & $\overrightarrow{0}$ & $\begin{array}{l}\tilde{0} \\
0 \\
0\end{array}$ & $\underset{\vec{i}}{\vec{J}}$ \\
\hline हैं & $\underset{\mathrm{C}}{\tilde{i}}$ & $\stackrel{?}{?}$ & ָे. & $\underset{⿱ 乛}{+}$ & $\tilde{\tilde{o}}$ & $\begin{array}{l}\overrightarrow{0} \\
0\end{array}$ & $\begin{array}{c}m \\
\infty \\
0 \\
0\end{array}$ & $\underset{\mathrm{i}}{\tilde{i}}$ & $\underset{i}{\stackrel{\Xi}{i}}$ & 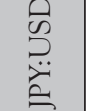 & 0 & $\overbrace{0}^{0}$ & $\begin{array}{l}0 \\
\dot{0}\end{array}$ & 0 & 0 & 0 & $\stackrel{?}{\stackrel{\sim}{\sim}}$ \\
\hline$\tilde{\sim}$ & $\stackrel{\infty}{\sim}$ & $\stackrel{\circ}{\circ}$ & $\begin{array}{l}8 \\
\dot{0}\end{array}$ & $\stackrel{20}{\circ}$ & $\stackrel{\widetilde{T}}{0}$ & $\exists$ & $\tilde{0}$ & $\underset{\sim}{\rightarrow}$ & ڤે & 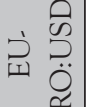 & 0 & $\overrightarrow{0}$ & $\begin{array}{l}\widetilde{\sigma} \\
\dot{0}\end{array}$ & $\begin{array}{l}\tau \\
\stackrel{0}{0} \\
0\end{array}$ & $\begin{array}{l}\overrightarrow{0} \\
0 \\
0\end{array}$ & 0 & $\begin{array}{l}\widetilde{\sigma} \\
\infty\end{array}$ \\
\hline 0 & o̊. & $\stackrel{0}{\circ}$ & $\tilde{O}$ & $\overrightarrow{0}$ & $\stackrel{2}{2}$ & $\begin{array}{l}0 \\
0 \\
0\end{array}$ & $\begin{array}{l}\infty \\
0 \\
0\end{array}$ & $\begin{array}{c}\vec{m} \\
\dot{n}\end{array}$ & $\overrightarrow{\stackrel{F}{+}}$ & 荀 & $\stackrel{\curvearrowright}{\sim}$ & ঐे & $\begin{array}{l}n \\
n \\
m \\
m\end{array}$ & 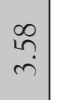 & $\ddot{m}$ & $\begin{array}{l}\vec{\infty} \\
\dot{m}\end{array}$ & $\stackrel{\text { ô. }}{0}$ \\
\hline : & ô. & $\begin{array}{c}\vec{\nabla} \\
\text { ஸ }\end{array}$ & $\underset{+}{+}$ & $\begin{array}{l}\infty \\
0 \\
0\end{array}$ & $\stackrel{ \pm}{ \pm}$ & $\begin{array}{l}0 \\
0 \\
0\end{array}$ & $\tilde{0}$ & $\begin{array}{c}\vec{n} \\
m\end{array}$ & $\overrightarrow{\stackrel{F}{f}}$ & & $\begin{array}{l}\stackrel{?}{\sim} \\
i\end{array}$ & $m$ & $\underset{\dot{f}}{\tilde{m}}$ & $\begin{array}{l}\text { in } \\
\text { nn }\end{array}$ & $\begin{array}{l}+ \\
\stackrel{m}{2}\end{array}$ & in. & $\tilde{O}$ \\
\hline$\tilde{O}$ & రి & 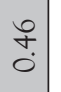 & $\begin{array}{l} \pm \\
0 \\
0\end{array}$ & $\begin{array}{l}\tilde{0} \\
0 \\
0\end{array}$ & $\begin{array}{l}n \\
n \\
0\end{array}$ & กิ & $\tilde{O}$ & $\stackrel{m}{m}$ & $\tilde{\tilde{r}}$ & & $\begin{array}{l}\overrightarrow{0} \\
i \\
i\end{array}$ & $\stackrel{\sim}{m}$ & $\begin{array}{l}0 \\
\dot{m}\end{array}$ & $\begin{array}{l}\infty \\
\infty \\
\infty \\
\end{array}$ & $\begin{array}{l}\infty \\
\stackrel{\infty}{0} \\
\dot{m}\end{array}$ & $\stackrel{0}{\dot{m}}$ & $\begin{array}{l}\infty \\
\stackrel{0}{0} \\
0\end{array}$ \\
\hline$\tilde{O}$ & $\stackrel{\text { ô. }}{0}$ & $\begin{array}{l}\infty \\
\stackrel{\infty}{0} \\
\stackrel{0}{0}\end{array}$ & : & $\begin{array}{l} \pm \\
\dot{0} \\
0\end{array}$ & $\stackrel{+}{\sim}$ & $\overrightarrow{\tilde{0}}$ & $\begin{array}{l} \pm \\
0 \\
0\end{array}$ & $\stackrel{n}{m}$ & $\underset{\forall}{\stackrel{t}{+}}$ & 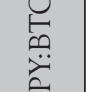 & $\begin{array}{c}\tilde{\sigma} \\
i\end{array}$ & $\stackrel{n}{\stackrel{i}{i}}$ & $\begin{array}{l}\infty \\
\infty \\
\dot{m}\end{array}$ & $\underset{\sim}{\stackrel{\sim}{\sim}}$ & $\underset{\dot{m}}{\vec{m}}$ & $\stackrel{\sim}{\sim}$ & $\begin{array}{l} \pm \\
\end{array}$ \\
\hline ¿̊. & $\stackrel{\infty}{\infty}$ & $\stackrel{7}{0}$ & $\begin{array}{l}\tilde{O} \\
\dot{0}\end{array}$ & $\begin{array}{l}\tilde{O} \\
\dot{0}\end{array}$ & $\stackrel{2}{\tilde{0}}$ & $\begin{array}{c}\tilde{0} \\
0\end{array}$ & $\begin{array}{l}\overrightarrow{0} \\
\dot{0}\end{array}$ & $\begin{array}{l}\vec{n} \\
i\end{array}$ & 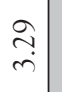 & 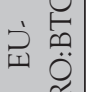 & $\stackrel{0}{\vec{n}}$ & in & $\stackrel{m}{m}$ & $\underset{\sim}{\stackrel{\sim}{\sim}}$ & $\vec{m}$ & $\stackrel{m}{m}$ & $\stackrel{\dddot{0}}{0}$ \\
\hline $\begin{array}{l}\overrightarrow{0} \\
0\end{array}$ & O̊. & $\begin{array}{l}\tilde{0} \\
0 \\
\end{array}$ & $\begin{array}{l}\overrightarrow{0} \\
\dot{0}\end{array}$ & $\begin{array}{l}\dot{t} \\
\dot{0} \\
\dot{0}\end{array}$ & $\overrightarrow{0}$ & ? & $\begin{array}{l}\tilde{0} \\
\dot{0}\end{array}$ & $\stackrel{m}{\vec{i}}$ & $\stackrel{\infty}{i}$ & 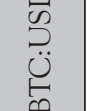 & $\begin{array}{l}\dot{0} \\
i \mathfrak{i n}\end{array}$ & 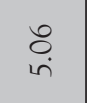 & $\begin{array}{l}\stackrel{n}{\hat{i}} \\
\dot{1}\end{array}$ & $\begin{array}{l}\infty \\
\infty \\
i \\
i\end{array}$ & 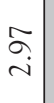 & $\stackrel{\infty}{i}$ & 0 \\
\hline 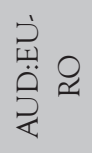 & 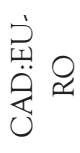 & 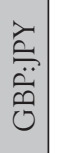 & 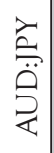 & 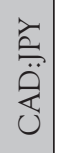 & 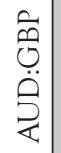 & 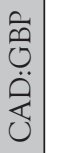 & 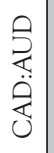 & 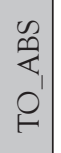 & $\begin{array}{l}\mathbb{E} \\
\overrightarrow{5} \\
\rho^{\prime} \\
\rho^{\prime}\end{array}$ & 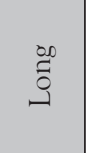 & 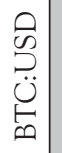 & 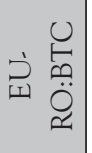 & 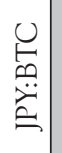 & 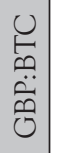 & 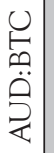 & 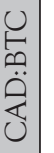 & 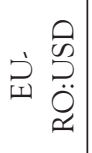 \\
\hline
\end{tabular}




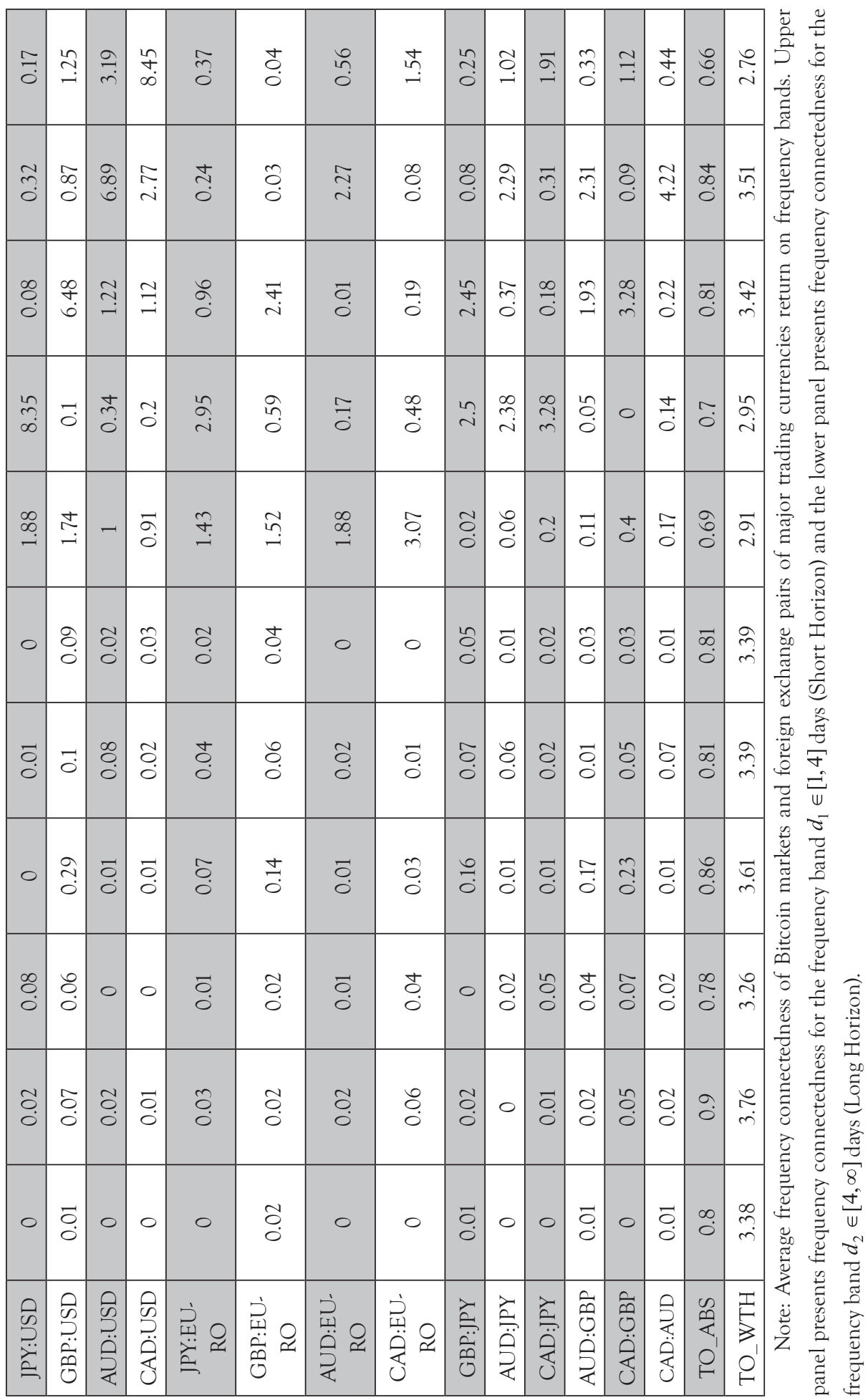




\begin{tabular}{|c|c|c|c|c|c|c|c|c|c|c|c|c|c|c|c|c|c|}
\hline $\begin{array}{l}\text { HLM } \\
\text { - } \text { WOYA }\end{array}$ & $\begin{array}{l}\infty \\
\stackrel{\infty}{n} \\
m\end{array}$ & $\stackrel{\nabla}{\sigma}$ & $\begin{array}{l}\infty \\
\infty \\
\end{array}$ & ले & $\begin{array}{l}\infty \\
\infty \\
\end{array}$ & $\begin{array}{l}\infty \\
\infty \\
\dot{m}\end{array}$ & $\vec{m}$ & $\stackrel{m}{m}$ & $\stackrel{n}{n}$ & $\begin{array}{l}\stackrel{0}{n} \\
m\end{array}$ & $\stackrel{\sim}{m}$ & $\begin{array}{l}n \\
\stackrel{n}{m}\end{array}$ & $\stackrel{\tilde{N}}{n}$ & $\dot{r}$ & $\stackrel{\sim}{+}$ & $\begin{array}{l}\tilde{\sigma} \\
\dot{n}\end{array}$ & $\stackrel{n}{n}$ \\
\hline $\begin{array}{c}\text { SqV } \\
- \text { WOYH }\end{array}$ & $\underset{\sim}{\sim}$ & $\underset{\sim}{\stackrel{N}{i}}$ & 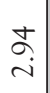 & $\hat{\widehat{\lambda}}$ & 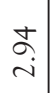 & 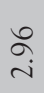 & $\tilde{\tilde{i}}$ & $\stackrel{\substack{\sim \\
\sim}}{\stackrel{2}{n}}$ & 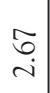 & 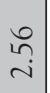 & $\begin{array}{l}J \\
i \\
i\end{array}$ & $\stackrel{\infty}{\stackrel{\infty}{\sim}}$ & $\stackrel{\sim}{\sim}$ & $\begin{array}{l}\hat{\sim} \\
i\end{array}$ & $\begin{array}{l}\stackrel{\sim}{i} \\
\overrightarrow{1}\end{array}$ & $\stackrel{\infty}{i}$ & $\begin{array}{l}\text { jo } \\
\text { i }\end{array}$ \\
\hline 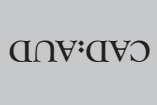 & $\hat{O}$ & $\hat{\circ}$ & $\begin{array}{l}n \\
0 \\
0\end{array}$ & $\begin{array}{l}+ \\
0 \\
0\end{array}$ & $\begin{array}{l}0 \\
0 \\
0\end{array}$ & ?ִ & $\begin{array}{l}\vec{T} \\
0\end{array}$ & $\begin{array}{l}\infty \\
0 \\
0\end{array}$ & $\begin{array}{l}\infty \\
\mathfrak{n} \\
0\end{array}$ & $\begin{array}{l}n \\
\stackrel{\infty}{\infty}\end{array}$ & $\stackrel{\infty}{\rightarrow}$ & $\stackrel{\overrightarrow{0}}{\circ}$ & $\overrightarrow{0}$ & $\begin{array}{l}\infty \\
\stackrel{+}{+} \\
\stackrel{+}{*}\end{array}$ & $\begin{array}{l}0 \\
\sim \\
\sim\end{array}$ & $\stackrel{\tilde{o}}{0}$ & $\underset{\sim}{\tilde{i}}$ \\
\hline 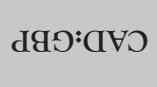 & 0 & $\begin{array}{l}\text { Jे } \\
\stackrel{0}{0}\end{array}$ & $\overrightarrow{0}$ & خે & \begin{tabular}{l}
$\circ$ \\
\hdashline \\
0
\end{tabular} & $\hat{o}$ & $\underset{-}{\approx}$ & $\stackrel{7}{\circ}$ & $\stackrel{\text { } \alpha}{\alpha}$ & $\stackrel{\infty}{\underset{0}{0}}$ & $\begin{array}{l}\vec{\sigma} \\
\dot{r}\end{array}$ & $\stackrel{\infty}{\rightleftarrows}$ & $\begin{array}{l}\bar{\sigma} \\
\dot{n}\end{array}$ & 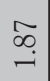 & $\begin{array}{l}+ \\
0 \\
0 \\
0\end{array}$ & $\begin{array}{l}\underset{+}{t} \\
\stackrel{+}{*}\end{array}$ & ठे. \\
\hline 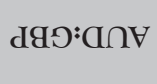 & $\begin{array}{l}\overrightarrow{0} \\
\dot{0}\end{array}$ & $\tilde{o}$ & $\begin{array}{l}2 \\
0 \\
0\end{array}$ & 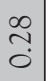 & 0 & $\begin{array}{l}\text { Oे } \\
0\end{array}$ & 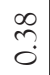 & ঙे. & \begin{tabular}{l}
\multirow{\sigma}{*}{} \\
in
\end{tabular} & $\stackrel{n}{\natural}$ & $\stackrel{\infty}{\rightarrow}$ & $\stackrel{?}{=}$ & $\begin{array}{l}\tilde{o} \\
\dot{n}\end{array}$ & $\underset{\infty}{=}$ & $\stackrel{\infty}{-}$ & $\begin{array}{l}\tilde{\sigma} \\
\dot{\gamma}\end{array}$ & $\overrightarrow{\mathrm{i}}$ \\
\hline XdI: đFO & $\begin{array}{c}\tilde{0} \\
\dot{0}\end{array}$ & Õ. & $\stackrel{ \pm}{ \pm}$ & oे. & $\stackrel{7}{\circ}$ & $\stackrel{2}{\circ}$ & $\begin{array}{l}\infty \\
m \\
0\end{array}$ & $\stackrel{n}{\beth}$ & $\tilde{o}$ & $\stackrel{\infty}{\underset{-}{-}}$ & $\begin{array}{l}0 \\
\infty \\
\infty\end{array}$ & $\underset{\infty}{\stackrel{0}{\infty}}$ & $\underset{\sim}{\mathbb{N}}$ & $\stackrel{\sim}{\sim}$ & $\begin{array}{l}n \\
\stackrel{2}{\sim}\end{array}$ & $\underset{\infty}{\vec{\infty}}$ & $\begin{array}{l}\stackrel{0}{+} \\
\stackrel{+}{0}\end{array}$ \\
\hline Xdl: $\mathrm{C} \cap \mathrm{V}$ & $\begin{array}{l}\overrightarrow{0} \\
\dot{0}\end{array}$ & $\stackrel{\overrightarrow{0}}{\circ}$ & $\begin{array}{l}\infty \\
0 \\
0\end{array}$ & $\begin{array}{l}+ \\
0 \\
0\end{array}$ & $\frac{n}{3}$ & $\begin{array}{l}\text { ¿ } \\
\text { Oे }\end{array}$ & $\begin{array}{l}2 \\
0 \\
0\end{array}$ & $\overbrace{\infty}^{+}$ & $\stackrel{\leftrightarrow}{\rightarrow}$ & $\begin{array}{c}\grave{J} \\
\sigma\end{array}$ & $\begin{array}{l}\circ \\
\infty \\
\dot{+}\end{array}$ & $\stackrel{\infty}{\stackrel{\infty}{\sim}}$ & $\begin{array}{l}0 \\
\stackrel{i}{ }\end{array}$ & $\stackrel{m}{\infty}$ & $\stackrel{\curvearrowright}{\grave{i}}$ & $\stackrel{\sim}{\sim}$ & $\stackrel{\widetilde{N}}{\Xi}$ \\
\hline Xdl:dGO & $\begin{array}{l}\tilde{O} \\
0 \\
0\end{array}$ & ¿̊. & $\begin{array}{l}\tilde{O} \\
0 \\
0\end{array}$ & 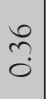 & $\stackrel{ \pm}{\stackrel{ \pm}{0}}$ & oे. & $\tilde{0}$ & $\begin{array}{l}\infty \\
\infty \\
\sigma\end{array}$ & ๗ે. & $\begin{array}{l}\widetilde{J} \\
\dot{0}\end{array}$ & શे. & $\stackrel{n}{m}$ & $\begin{array}{l}\text { ț } \\
\dot{0} \\
\dot{0}\end{array}$ & $\stackrel{m}{0}$ & $\begin{array}{l}\tilde{T} \\
\tilde{0}\end{array}$ & $\stackrel{\infty}{\stackrel{(}{\leftrightarrows}}$ & $\stackrel{?}{\sim}$ \\
\hline $\begin{array}{c}\text { O\& } \\
-\cap a ः \text { đYO }\end{array}$ & $\begin{array}{l}\tilde{O} \\
0\end{array}$ & oे. & $\begin{array}{l}0 \\
0 \\
0\end{array}$ & $\begin{array}{l}\tilde{0} \\
0\end{array}$ & $\stackrel{2}{2} 0$ & б. & $\stackrel{0}{\overrightarrow{0}}$ & $\tilde{\xi}$ & $\begin{array}{l}\widetilde{\sigma} \\
0 \\
0\end{array}$ & $\dot{\circ}$ & $\underset{\Xi}{ \pm}$ & $\stackrel{\sim}{\stackrel{\sim}{i}}$ & $\begin{array}{l}\stackrel{0}{ } \\
\dot{r}\end{array}$ & & $\begin{array}{l}\stackrel{0}{\sim} \\
\stackrel{\sim}{\sim}\end{array}$ & $\stackrel{2}{\circ}$ & $\widetilde{-}$ \\
\hline 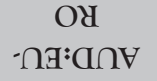 & $\begin{array}{l}\tilde{O} \\
\dot{0}\end{array}$ & $\begin{array}{l}\infty \\
\stackrel{0}{0} \\
0\end{array}$ & $\begin{array}{l}\tilde{0} \\
0\end{array}$ & $\begin{array}{l}0 \\
0 \\
0\end{array}$ & $\begin{array}{l}+ \\
0 \\
0\end{array}$ & $\begin{array}{l}\text { ¿ } \\
\text { Oे }\end{array}$ & $\begin{array}{l}\stackrel{2}{\sim} \\
i n\end{array}$ & $\hat{n}$ & oे & $\infty$ & $\stackrel{\sim}{\sim}$ & $\underset{\mathrm{i}}{\vec{i}}$ & $\stackrel{\sim}{+}$ & $\begin{array}{l}\tilde{\sigma} \\
\dot{0}\end{array}$ & సે. & $=$ & $\overbrace{}^{n}$ \\
\hline $\begin{array}{c}\text { O४ } \\
\text {-ก马ःdgЭ }\end{array}$ & $\begin{array}{l}\tilde{O} \\
O \\
0\end{array}$ & ठ̊ & $\begin{array}{l}\text { J } \\
0 \\
0\end{array}$ & $\stackrel{\simeq}{0}$ & $\begin{array}{l}8 \\
0 \\
0\end{array}$ & ֶ̊ & $\begin{array}{l}\stackrel{0}{+} \\
\stackrel{+}{*}\end{array}$ & 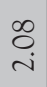 & $\begin{array}{l}\vec{\infty} \\
0\end{array}$ & $\vec{Z}$ & $\frac{2}{0}$ & $\stackrel{\Xi}{0}$ & $\begin{array}{l}\vec{\nabla} \\
\vec{\sim}\end{array}$ & $\underset{m}{=}$ & $\stackrel{2}{\underset{+}{+}}$ & 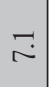 & $\stackrel{\infty}{\underset{I}{\leftrightarrows}}$ \\
\hline $\begin{array}{c}\text { O\& } \\
-\cap \exists: X d l\end{array}$ & $\begin{array}{l}\tilde{O} \\
0 \\
0\end{array}$ & $\frac{n}{0}$ & $\stackrel{\sigma}{0}$ & $\tilde{0}$ & 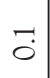 & $\begin{array}{l}\infty \\
\stackrel{0}{0} \\
0\end{array}$ & $\begin{array}{l}+ \\
\infty \\
\text { in }\end{array}$ & જે & 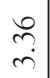 & $\begin{array}{l}\tilde{\sigma} \\
\dot{0}\end{array}$ & $\stackrel{\overbrace{}}{\stackrel{\imath}{-}}$ & 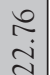 & $\underset{0}{ \pm}$ & $\stackrel{\infty}{\underset{\sim}{\sim}}$ & $\stackrel{n}{n}$ & $\stackrel{\text { O઼ }}{\circ}$ & 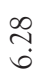 \\
\hline
\end{tabular}




\begin{tabular}{|c|c|c|c|c|c|c|c|c|c|c|c|c|c|c|c|c|c|c|c|c|}
\hline ஸे & $\stackrel{+}{m}$ & $\stackrel{m}{m}$ & $\tilde{i}$ & & $\underset{\substack{d \\
i}}{ }$ & 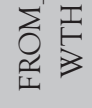 & 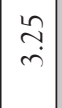 & กี & $\begin{array}{l}0 \\
\stackrel{0}{0} \\
\end{array}$ & $\stackrel{n}{m}$ & $\stackrel{\tilde{n}}{n}$ & $\underset{\dot{m}}{+}$ & $\stackrel{\infty}{m}$ & $\begin{array}{l}\stackrel{0}{\stackrel{n}{n}} \\
\text {. }\end{array}$ & $\stackrel{\sim}{\sim}$ & $\tilde{m}$ & 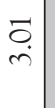 & in & $\stackrel{\sim}{m}$ & $\stackrel{\circ}{\dot{m}}$ \\
\hline$\underset{\sim}{\stackrel{N}{i}}$ & 公 & 岌 & $\begin{array}{l}8 \\
i \\
i\end{array}$ & $\begin{array}{l}\text { जิ } \\
\text { in }\end{array}$ & & $\begin{array}{ll}\sum_{0} & n \\
0 & \frac{1}{4}\end{array}$ & $\underset{\mathfrak{O}}{\mathfrak{0}}$ & $\begin{array}{l}+ \\
\stackrel{\infty}{0} \\
0\end{array}$ & $\begin{array}{c}\tilde{D} \\
0 \\
0\end{array}$ & $\begin{array}{c}\widetilde{\infty} \\
0 \\
0\end{array}$ & 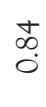 & $\begin{array}{c}\tilde{D} \\
0 \\
0\end{array}$ & $\begin{array}{l}\stackrel{゚}{\circ} \\
\stackrel{0}{*}\end{array}$ & $\begin{array}{c}\text { के } \\
0 \\
0\end{array}$ & $\begin{array}{l}\stackrel{0}{0} \\
\stackrel{0}{0}\end{array}$ & $\begin{array}{l}\infty \\
0 \\
0\end{array}$ & $\underset{0}{\stackrel{N}{0}}$ & 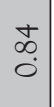 & $\begin{array}{l}\stackrel{゚}{0} \\
\vdots\end{array}$ & $\begin{array}{l}\infty \\
\infty \\
0 \\
0\end{array}$ \\
\hline$\stackrel{\overbrace{}}{\text { ?̊. }}$ & $\stackrel{\stackrel{\sim}{f}}{\stackrel{f}{*}}$ & $\stackrel{2}{\stackrel{\sim}{\sim}}$ & $\frac{\tilde{\sigma}}{\dot{m}}$ & $\stackrel{F}{\stackrel{F}{-}}$ & 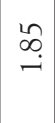 & 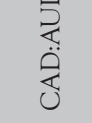 & $\overrightarrow{0}$ & $\begin{array}{l}\tilde{O} \\
\text { Oे }\end{array}$ & 0 & 0 & $\begin{array}{l}\overrightarrow{0} \\
\end{array}$ & $\begin{array}{l}\overrightarrow{0} \\
\circ\end{array}$ & ठे. & $\begin{array}{l}\infty \\
\stackrel{0}{0} \\
\stackrel{0}{0}\end{array}$ & $\begin{array}{l} \pm \\
0 \\
0\end{array}$ & $\stackrel{n}{i}$ & $\begin{array}{l}\infty \\
\infty \\
0 \\
0\end{array}$ & 0 & $=$ & 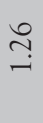 \\
\hline$\stackrel{\circ}{\rightarrow}$ & $\begin{array}{l}\infty \\
\infty \\
\stackrel{0}{0} \\
\end{array}$ & $\begin{array}{l}\stackrel{\infty}{d} \\
\underset{\sim}{N}\end{array}$ & $\stackrel{n}{\hat{i}}$ & 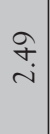 & $\stackrel{\widetilde{N}}{\sim}$ & 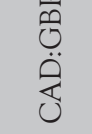 & $\overrightarrow{\tilde{O}}$ & $\stackrel{\text { ô }}{\circ}$ & $\begin{array}{l}\tilde{0} \\
\dot{0}\end{array}$ & $\vec{\circ}$ & 官 & 0 & 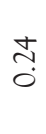 & $\begin{array}{l}\overrightarrow{0} \\
\dot{0}\end{array}$ & $\begin{array}{l}\tilde{\sigma} \\
\stackrel{i}{i}\end{array}$ & $\begin{array}{l}7 \\
0 \\
0\end{array}$ & 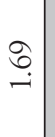 & 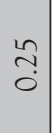 & $\stackrel{\circ}{\stackrel{-}{-}}$ & $\begin{array}{l}\vec{n} \\
\text { مे }\end{array}$ \\
\hline ?ֶ. & $\stackrel{\text { ڤ }}{\vec{\sim}}$ & $\stackrel{\widetilde{D}}{=}$ & 苂 & $\begin{array}{l}0 \\
\dot{i}\end{array}$ & $\stackrel{\sim}{\sim}$ & 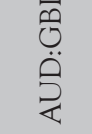 & 0 & $\overrightarrow{0}$ & 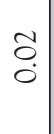 & $\overrightarrow{0}$ & 0 & $\begin{array}{l}\overrightarrow{0} \\
\dot{0}\end{array}$ & : & $\begin{array}{l}\tilde{0} \\
0 \\
0\end{array}$ & $\underset{\sim}{\stackrel{\sim}{\sim}} \mid$ & کَ & $\tilde{o}$ & $\begin{array}{l}\vec{\pi} \\
0\end{array}$ & 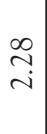 & $\stackrel{2}{\underset{\sim}{\sim}}$ \\
\hline 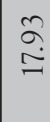 & $\begin{array}{l}\infty \\
\stackrel{0}{0}\end{array}$ & $\stackrel{\overbrace{}}{\Im}$ & $\begin{array}{l}\infty \\
\underset{i}{i}\end{array}$ & $\stackrel{\widetilde{r}}{\tilde{m}}$ & 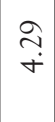 & 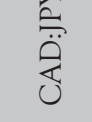 & 0 & 0 & $\begin{array}{l}\tilde{O} \\
0 \\
0\end{array}$ & ठ․ & ָे. & $\begin{array}{l}\tilde{o} \\
0 \\
0\end{array}$ & ô. & $\overrightarrow{\tilde{r}}$ & 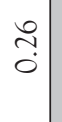 & î. & $\begin{array}{l}\hat{b} \\
i\end{array}$ & $\vec{m}$ & $\begin{array}{l}\infty \\
i \\
? \\
?\end{array}$ & 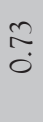 \\
\hline$\stackrel{\widetilde{\sigma}}{\dot{\Xi}}$ & ले & $\stackrel{n}{0}$ & $\stackrel{\widetilde{F}}{+}$ & $\stackrel{m}{m}$ & 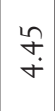 & 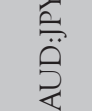 & $\overrightarrow{0}$ & 0 & $\begin{array}{l}\tilde{O} \\
0 \\
0\end{array}$ & ¿্口. & 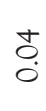 & $\begin{array}{l}0 \\
0 \\
0\end{array}$ & 0 & $\vec{m}$ & 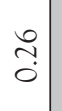 & 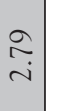 & $\underset{\sim}{\tilde{r}}$ & $\begin{array}{c}\tilde{0} \\
\text { r. }\end{array}$ & $\tilde{\tilde{o}}$ & $\stackrel{+}{i}$ \\
\hline $\begin{array}{l}0 \\
+ \\
\infty\end{array}$ & $\stackrel{\text { fo }}{i n}$ & $\begin{array}{l}\text { के } \\
\text { in }\end{array}$ & $\begin{array}{l}\tilde{0} \\
\dot{0}\end{array}$ & 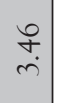 & $\begin{array}{l}\stackrel{+}{\stackrel{+}{+}} \\
\dot{\gamma}\end{array}$ & 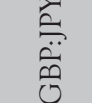 & $\begin{array}{l}\overrightarrow{0} \\
\dot{0}\end{array}$ & ¿.: & 0 & $\stackrel{7}{\circ}$ & $\begin{array}{l}\text { D. } \\
\text { Oे }\end{array}$ & $\begin{array}{l}\tilde{\sigma} \\
0 \\
0\end{array}$ & ¿̂. & $\begin{array}{l}\tilde{\sigma} \\
\text { r. }\end{array}$ & 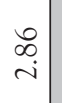 & 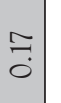 & $\underset{⿱}{\Delta}$ & $\begin{array}{l}\infty \\
\stackrel{m}{m}\end{array}$ & $\tilde{\ddot{m}}$ & lo \\
\hline$\stackrel{\infty}{n}$ & : & 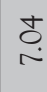 & $\begin{array}{l}\infty \\
\infty \\
\dot{n}\end{array}$ & 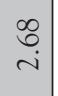 & $\tilde{n}$ & 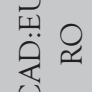 & $\overrightarrow{0}$ & ô. & 0 & 0 & I̋ & $\tilde{O}$ & $\stackrel{\infty}{\stackrel{i}{i}}$ & $\begin{array}{c}\tilde{\infty} \\
\infty \\
0\end{array}$ & 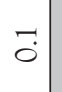 & $\tilde{\tilde{o}}$ & $\stackrel{\infty}{\underset{i}{i}}$ & t. & 恶 & $\underset{\sim}{\tilde{m}}$ \\
\hline$\stackrel{9}{-}$ & 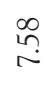 & $\stackrel{\widetilde{\infty}}{\rightarrow}$ & f. & $\begin{array}{l}n \\
\stackrel{i}{i}\end{array}$ & $\overrightarrow{\dot{r}}$ & 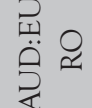 & 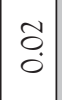 & : & 0 & 0 & ${ }_{0}^{t}$ & $\begin{array}{l}\tilde{O} \\
\text { O. }\end{array}$ & $\stackrel{t}{\rightarrow}$ & $\begin{array}{l}\text { ff } \\
0 \\
0\end{array}$ & $\begin{array}{l}\tilde{5} \\
0 \\
0\end{array}$ & 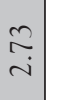 & ถે. & $\tilde{n}_{0}^{n}$ & $\stackrel{\widetilde{T}}{\text {. }}$ & $\stackrel{\sim}{\text { ô }}$ \\
\hline$\stackrel{t}{\mathrm{t}}$ & $\stackrel{\sim}{i n}$ & 究 & $\tilde{0}$ & $\sim$ & $\underset{\tilde{r}}{\mathrm{i}}$ & 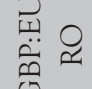 & ¿ّ. & 0 & $\begin{array}{l}\tilde{O} \\
\dot{0}\end{array}$ & $\stackrel{?}{\circ}$ & ¿. & $\begin{array}{l}2 \\
0 \\
0 \\
0\end{array}$ & مِّ & $\stackrel{\rightarrow}{\circ}$ & $\vec{i}$ & $\begin{array}{l}2 \\
0 \\
0 \\
0\end{array}$ & $\begin{array}{l}2 \\
\stackrel{2}{0} \\
0\end{array}$ & $\stackrel{\widetilde{O}}{0}$ & $\stackrel{\circ}{\stackrel{\infty}{2}}$ & $\stackrel{\infty}{+}$ \\
\hline$\stackrel{?}{\stackrel{2}{\pi}}$ & هి & مِ & \begin{tabular}{l}
\multirow{2}{0}{} \\
$\dot{0}$
\end{tabular} & $\stackrel{\sim}{\stackrel{2}{\sim}}$ & $\stackrel{\circ}{i}$ & 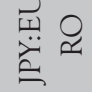 & 0 & రి & $\begin{array}{l}\tilde{O} \\
\dot{0}\end{array}$ & $\begin{array}{l}\text { Oे. } \\
\stackrel{0}{0}\end{array}$ & ¿. & $\overrightarrow{0}$ & $\stackrel{\sim}{\mathrm{N}}$ & $\stackrel{\sim}{\stackrel{\sim}{\sim}}$ & $\begin{array}{l}\stackrel{2}{0} \\
\stackrel{0}{*}\end{array}$ & $\stackrel{2}{\circ}$ & $\tilde{O}$ & $\stackrel{n}{\sim}$ & $\stackrel{ \pm}{*}$ & $\underset{0}{\mathbb{N}}$ \\
\hline
\end{tabular}




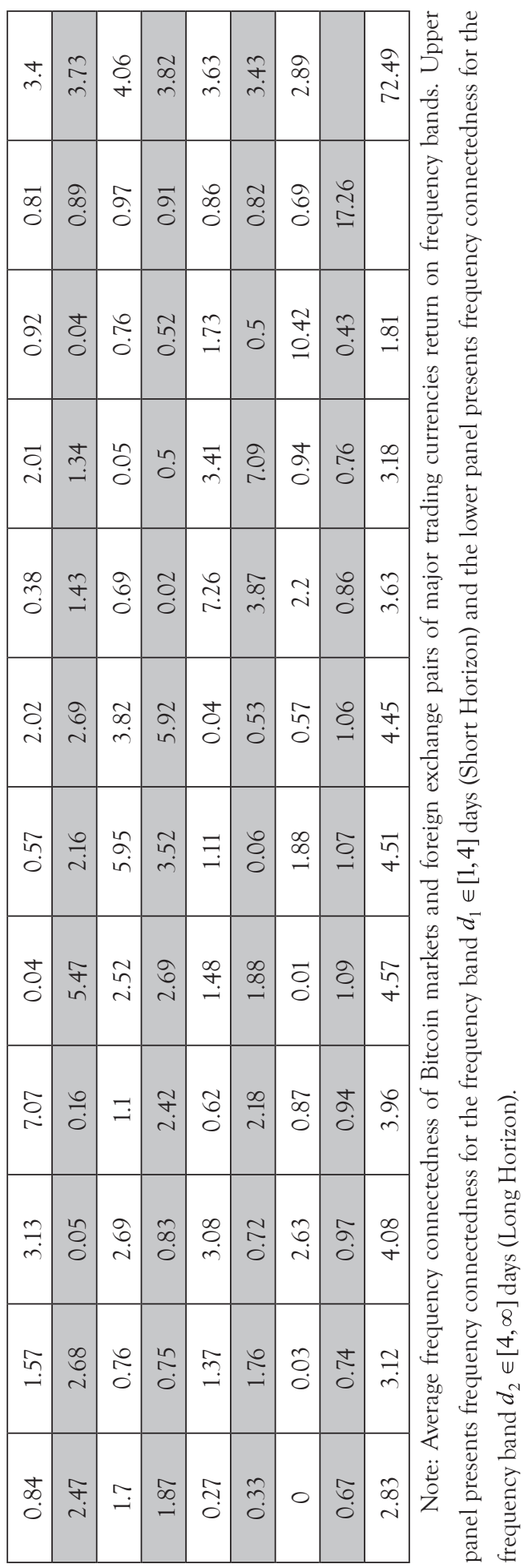




\subsection{Frequency connectedness}

The frequency connectedness for returns of Bitcoin markets and foreign exchange pairs dominated in six major trading currencies is depicted in table 4 and figure 4 .

The static return frequency connectedness results (table 4) indicates that return spillover for Bitcoin markets and foreign exchange pairs dominated in six major trading currencies is dominated at short frequencies. In the return spillover frequency connectedness (Table 4) it is found that at short frequency highest absolute and within connectedness from other is shown by GBP:BTC and lowest absolute and within connectedness from other is depicted by CAD:AUD. Whereas, highest absolute and within connectedness to others at short frequency is shown by GBP:JPY and lowest absolute and within connectedness to others is shown by CAD:AUD. At long horizon the highest absolute and within connectedness from other is revealed by AUD:JPY and lowest absolute and within connectedness from others is shown by CAD:AUD. Whereas, at highest absolute and within connectedness to others at long horizon is depicted by GBP:JPY and lowest absolute and within connectedness to others at long horizon is depicted by CAD:AUD.

The time varying dynamic frequency connectedness analysis for return spillover at short and long frequency reveals that return spillover among the Bitcoin markets and foreign exchange pairs dominated in six major trading currencies is dominated at short frequencies and the major spillover among them occurs within four days.
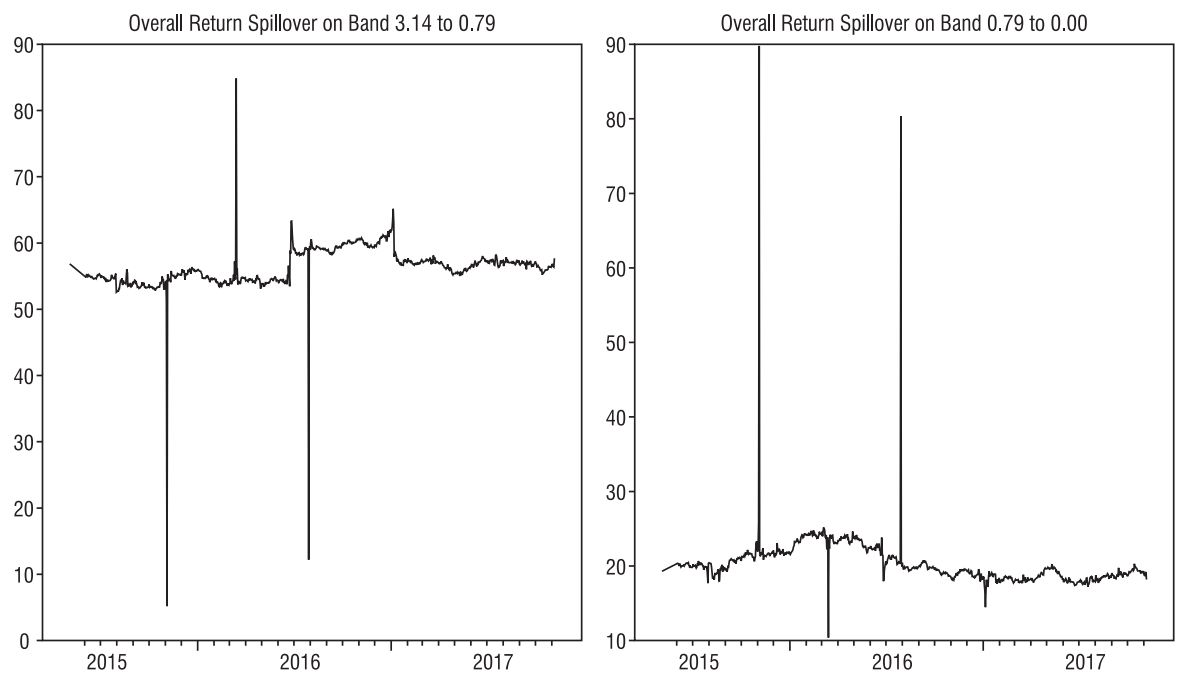

Figure 4: Dynamic returns' frequency connectedness of Bitcoin markets and foreign exchange pairs dominated in six major trading currencies on frequency bands. Left plot presents frequency connectedness for the frequency band $d_{1} \in[1,4]$ days (Short Horizon) and the right plot presents frequency connectedness for the frequency band $d_{2} \in[4, \infty]$ days (Long Horizon). 


\section{Conclusion}

In this study, we analysed the dynamic nature of return spillover between the Bitcoin markets and the foreign exchange pairs dominated in six major trading currencies. The spillover index methodology of Diebold and Yilmaz (2012), SAM methodology of Barunik, Kocenda and Vacha (2017), and frequency connectedness methodology of Barunik and Krehlik (2018) are applied for analysis. The findings of Diebold and Yilmaz (2012) indicated that there exist low return spillover between Bitcoin markets and foreign exchange markets. Evidence indicates significant intra-market return spillover in Bitcoin and foreign exchange markets. The patterns of return spillover exhibit similar trend over time and provide evidence of its sensitivity to global economic and financial events. However, asymmetric nature of return spillover among the Bitcoin markets and foreign exchange pairs of six major trading currencies is also identified. It is revealed that positive and negative, returns and volatilities do not create similar impact on other markets in terms of spillover. Moreover, it is also found that return spillover among the Bitcoin markets and the foreign exchange pairs denominated in six major trading currencies is dominated at short horizon and significant spillover occurs within 4 days of an event.

The evidence for low integration of Bitcoin markets with the foreign exchange market provide significant implication for portfolio diversification and risk minimization. Investors in the foreign exchange market can use Bitcoins investment as a hedge against the risk associated with the foreign exchange market. In the similar fashion, the Bitcoin investors can add foreign exchange investment into their portfolios to diversify their risk associated with Bitcoin investments. Portfolio managers and speculative investors can utilize the information to create their minimum risk portfolio by diversifying their investment among foreign exchange and Bitcoin markets. This study also provides a clue for future work to analyse the integration and interconnectedness of crypto-currencies with other financial markets will provide valuable insight into the portfolio diversification benefits that crypto-currencies' investment offer.

Investment managers have experienced losses due to the presence of unpredicted volatility in the prices of assets. With increasing acceptance of Bitcoin, the price volatility of Bitcoin is experiencing stability. Therefore, for investment managers Bitcoin has the potential to be a portfolio diversifier against uncertainty in the financial markets and monetary system. The evidence for low integration of Bitcoin markets with the foreign exchange market provide significant implication for portfolio diversification and risk minimization. Investors in the foreign exchange market can use Bitcoins investment as a diversifier against the risk associated with the foreign exchange market. In the similar fashion, the Bitcoin investors can add foreign exchange investment into their portfolios to diversify their risk associated with Bitcoin 
investments. Portfolio managers and speculative investors can utilize the information to create their minimum risk portfolio by diversifying their investment among foreign exchange and Bitcoin markets.

\section{References}

Barunik, J., \& Krehlik, T. (2016). Measuring the frequency dynamics of financial and macroeconomic connectedness (FinMap Working Paper No. 54). Paris: Financial Distortions and Macroeconomic Performance: Expectations, Constraints and Interaction of Agents. Retrieved from https://www.econstor.eu/ bitstream/10419/125824/1/845696696.pdf

Barunik, J., \& Křehlik, T. (2018). Measuring the frequency dynamics of financial connectedness and systemic risk. Journal of Financial Econometrics, 16(2), 271-296.

Barunik, J., Kocenda, E., \& Vacha, L. (2015). Volatility spillovers across petroleum markets. Energy Journal, 36(3), 309-329

Barunik, J., Kočenda, E., \& Vácha, L. (2017). Asymmetric volatility connectedness on the Forex market. Journal of International Money and Finance, 77, 39-56.

Basu, M. R. (2002). Financial contagion and investor "learning": An empirical investigation (IMF Working Paper No. 02/218). Washington D.C.: International Monetary Fund. Retrieved from https://www. imf.org/external/pubs/ft/wp/2002/wp02218.pdf

Bollerslev, T. (1990). Modelling the coherence in short-run nominal exchange rates: A multivariate generalized ARCH model. The Review of Economics and Statistics, 72(3), 498-505.

Bordo, M. D. \& Murshid, A. P. (2001). Are financial crises becoming more contagious?: What is the historical evidence on contagion? In S. Claessens \& K. J. Forbes (Eds.), International financial contagion. (pp. 367-403). New York, NY: Springer Science+Business Media.

Bouri, E., Molnár, P., Azzi, G., Roubaud, D., \& Hagfors, L. I. (2017). On the hedge and safe haven properties of bitcoin: Is it really more than a diversifier? Finance Research Letters, 20, 192-198.

Bouri, E., Gupta, R., Tiwari, A. K., \& Roubaud, D. (2017). Does bitcoin hedge global uncertainty? Evidence from wavelet-based quantile-in-quantile regressions. Finance Research Letters, 23, 87-95.

Brandvold, M., Molnár, P., Vagstad, K., \& Valstad, O. C. A. (2015). Price discovery on Bitcoin exchanges. Journal of International Financial Markets, Institutions and Money, 36, 18-35.

Brière, M., Oosterlinck, K., \& Szafarz, A. (2015). Virtual currency, tangible return: Portfolio diversification with bitcoin. Journal of Asset Management, 16(6), 365-373.

Brouwer, G. (2005). Monetary and financial integration in Asia: Empirical evidence and issues. In Asian Development Bank (Ed.), Asia economic cooperation and integration: Progress, Prospects, Challenges. (pp. 269-293). Manila: Asian Development Bank. 
Cheah, E. T., \& Fry, J. (2015). Speculative bubbles in bitcoin markets? An empirical investigation into the fundamental value of bitcoin. Economics Letters, 130, 32-36.

Cheung, A., Roca, E., \& Su, J. J. (2015). Crypto-Currency bubbles: An application of the Phillips-Shi-Yu (2013) methodology on Mt. Gox bitcoin prices. Applied Economics, 47, 2348-2358.

Ciaian, P., Rajcaniova, M., \& Kancs, D. A. (2016). The economics of bitcoin price formation. Applied Economics, 48(19), 1799-1815. doi:10.1080/00036846.2015.1109038

Clark, T. E., \& West, K. D. (2007). Approximately normal tests for equal predictive accuracy in nested models. Journal of econometrics, 138(1), 291-311.

Dastgir, S., Demir, E., Downing, G., Gozgor, G., \& Lau, C. K. M. (2018). The causal relationship between bitcoin attention and bitcoin returns: Evidence from the Copula-Based Granger causality test. Finance Research Letters, 28, 160-164. doi:10.1016/j.frl.2018.04.019

Dew-Becker, I. \& Giglio, S. (2016). Asset pricing in the frequency domain: Theory and empirics. The Review of Financial Studies, 29(8), 2029-2068.

Diebold, F. X. \& Yilmaz, K. (2009). Measuring financial asset return and volatility spillovers, with application to global equity markets. The Economic Journal, 119(534), 158-171.

Diebold, F. X., \& Yilmaz, K. (2012). Better to give than to receive: Predictive directional measurement of volatility spillovers. International Journal of Forecasting, 28(1), 57-66.

Dyhrberg, A. H. (2016a). Bitcoin, gold and the dollar-A GARCH volatility analysis. Finance Research Letters, 16, 85-92. doi:10.1016/j.frl.2015.10.008

Dyhrberg, A. H. (2016b). Hedging capabilities of bitcoin. Is it the virtual gold? Finance Research Letters, $16,139-144$.

Engle, R. F. \& Kroner, K. F. (1995). Multivariate simultaneous generalized ARCH. Econometric theory, 11(01), 122-150.

Engle, R. F. \& Sheppard, K. (2001). Theoretical and empirical properties of dynamic conditional correlation multivariate GARCH (NBER Working Paper No. w8554). Cambridge, MA: National Bureau of Economic Research. Retrieved from https://www.nber.org/system/files/working_papers/w8554/w8554.pdf

Forbes, K. J. \& Rigobon, R. (2002). No contagion, only interdependence: Measuring stock market comovements. The Journal of Finance, 57(5), 2223-2261.

Fry, J., \& Cheah, E. T. (2016). Negative bubbles and shocks in cryptocurrency markets. International Review of Financial Analysis, 47, 343-352.

Glaser, F., Zimmermann, K., Haferkorn, M., Weber, M., \& Siering, M. (2014). Bitcoin-Asset or currency? Revealing users' hidden intentions [Paper presentation]. 22nd European Conference on Information Systems, Tel Aviv, Israel. Available at SSRN: https://ssrn.com/abstract=2425247 
Gulzar, S., Jan, U., E्F Afzal, B. (2019). Global financial crisis and its implications on the performance of financial sector of Pakistan. City University Research Journal, 9(2), 422-434.

Gulzar, S., Kayani, G. M., Xiaofeng, H., Ayub, U., \& Rafique, A. (2019). Financial cointegration and spillover effect of global financial crisis: A study of emerging Asian financial markets. Economic Research-Ekonomska Istraživanja, 32(1), 187-218.

Ho, N. W. (2009). Financial integration: Concepts and impacts. Macao Monetary Research Bulletin, 2009(10), 69-84.

Kerner, S. (2014, February 2). Why Marc Andreessen is bullish on bitcoin. Eweek. https://www.eweek. $\mathrm{com} /$ cloud/why-marc-andreessen-is-bullish-on-bitcoin

Koop, G., Pesaran, M. H., \& Potter, S. M. (1996). Impulse response analysis in non-linear multivariate models. Journal of Econometrics, 74(1), 119-147.

Koutmos, D. (2018). Return and volatility spillovers among cryptocurrencies. Economics Letters, 173, $122-127$.

Kristoufek, L. (2015). What are the main drivers of the bitcoin price? Evidence from wavelet coherence analysis. PLOS One, 10(4), 1-15. doi:10.1371/journal.pone.0123923

Kurihara, Y. \& Fukushima, A. (2017). The market efficiency of bitcoin: A weekly anomaly perspective. Journal of Applied Finance and Banking, 7(3), 57-64.

Markowitz, H. (1952). Portfolio selection. The Journal of Finance, 7(1), 77-91.

Molodtsova, T. \& Papell, D. H. (2009). Out-of-sample exchange rate predictability with Taylor rule fundamentals. Journal of international economics, 77(2), 167-180.

Narayan, P. K., Narayan, S., Rahman, R. E., \& Setiawan, I. (2018). Bitcoin price growth and Indonesia's monetary system. Emerging Markets Review, 38, 364-376.

Pesaran, M. H. \& Timmermann, A. (2002). Market timing and return prediction under model instability. Journal of Empirical Finance, 9(5), 495-510.

Pesaran, M. H. \& Shin, Y. (1998). Generalized impulse response analysis in linear multivariate models. Economics Letters, 58(1), 17-29.

Phillips, P. C. \& Yu, J. (2011). Dating the timeline of financial bubbles during the subprime crisis. Quantitative Economics, 2(3), 455-491.

Qarni, M. O. \& Gulzar, S. (2019). Intra-EMU and non-EMU, EU stock markets' return spillover: Evidence from ESDC. Empirica, 47, 543-577. doi:10.1007/s10663-019-09437-6

Qarni, M. O., Gulzar, S., Fatima, S. T., Khan, M. J., \& Shafi, K. (2019). Inter-Markets volatility spillover in U.S. bitcoin and financial markets. Journal of Business Economics and Management, 20(4), 694 714. doi:10.3846/jbem.2019.8316 
Qarni, M. \& Gulzar, S. (2018). Volatility spillover effects of Shanghai Stock Exchange crash on the stock markets of its major trading partners. Business EF Economic Review, 10(3), 1-20.

Richardson, V. (2014). Currency kings. Entrepreneur, 42, 40.

Urquhart, A. \& Zhang, H. (2018). Is bitcoin a hedge or safe-haven for currencies? An intraday analysis. Available at SSRN: https://ssrn.com/abstract $=3114108$ 